\title{
Efeitos dissipativos em Mecânica \\ Celeste modelados por corpos pseudo-rígidos
}

\author{
Lucas Ruiz dos Santos
}

TESE APRESENTADA

$\mathrm{AO}$

Instituto De MatemáticA E EstatísticA

DA

Universidade DE SÃO PAUlo

PARA

OBTENÇÃO DO TÍTULO

$\mathrm{DE}$

DOUTOR EM CIÊNCIAS

Programa: Pós graduação em Matemática Aplicada

Orientador: Prof. Dr. Clodoaldo Grotta Ragazzo

Durante o desenvolvimento deste trabalho o autor recebeu auxílio financeiro do CNPq

São Paulo, 21 de dezembro de 2015 


\section{Efeitos dissipativos em Mecânica \\ Celeste modelados por corpos pseudo-rígidos}

Esta dissertação contém as correções e alterações sugeridas pela Comissão Julgadora durante a defesa realizada por Lucas Ruiz dos Santos em 23/11/2015.

O original encontra-se disponível no Instituto de Matemática e Estatística da Universidade de São Paulo.

Comissão Julgadora:

- Prof. Dr. Clodoaldo Grotta Ragazzo (orientador) - IME-USP

- Prof. Dr. Pedro Antonio Santoro Salomão - IME-USP

- Prof. Dr. Sylvio Ferraz Mello - IAG-USP

- Prof. Dr. Luis Fernando de Osório Mello - UNIFEI

- Profa. Dra. Tatiana Alexandrovna Michtchenko - IAG-USP 


\section{Agradecimentos}

Considerei esta uma ocasião apropriada para agradecer às pessoas que conviveram comigo durante estes anos acadêmicos e que também possibilitaram que eu chegasse até aqui.

Gostaria de agradecer a minha família que nunca mediu esforços para me apoiar, e em especial à minha mãe, Maria de Fátima, que até nestes últimos anos de minha formação se preocupou para que eu desse prioridade aos estudos.

Na universidade, agradeço em especial a alguns professores que, para mim, foram muito além de suas obrigações como docentes, os quais hoje considero como amigos. Ao prof. Renato Klippert, por toda sua atenção e seriedade nos cruciais anos iniciais de minha graduação. Ao prof. José Augusto Baêta, que nos mostrou e mostra o quão bela é a Matemática e que fez despertar em mim e inúmeros de seus alunos interesse e respeito por esta disciplina, deixando claro o quanto somos imprecisos ao formular nossos argumentos e também quão necessárias são tais imprecisões em nosso desenvolvimento. Ao prof. Luis Fernando Mello, que desde os tempos de minha iniciação científica até esta defesa ofereceu apoio irrestrito, contribuindo muito para a maneira com que encaro a atividade científica séria. Ao prof. Clodoaldo pelos agradáveis anos de orientação, mestrado e doutorado, levantando questões interessantes e gerando discussões que me fizeram progredir muito.

Agradeço também aos professores Sylvio e Tatiana pelas várias sugestões e pela bibliografia sugerida, a qual foi essencial no entendimento do problema.

Também fica meu agradecimento aos meus colegas de estudo do IME-USP, Luis Fernando, Gabriel, Max, e meus colegas da UNIFEI-MG. Em especial, no desenvolvimento deste trabalho, agradeço ao Antonio Carlos Fernandes e ao Denis Braga.

Agradeço especialmente a minha noiva, Fernanda, por sempre oferecer uma palavra de apoio nos momentos difíceis, mesmo estando boa parte do tempo distantes. 


\section{Resumo}

Ruiz, L. S. Efeitos dissipativos em Mecânica Celeste modelados por corpos pseudorígidos. 2015. 81f. Tese de doutorado - Instituto de Matemática e Estatística, Universidade de São Paulo, São Paulo, 2015.

O presente trabalho dedica-se a uma modelagem da interação entre corpos celestes, em regime Newtoniano, levando-se em consideração as influências que suas deformações e viscosidades internas exercem sobre seus movimentos orbitais e suas velocidades angulares. A abordagem adotada é uma variação do conhecido problema do corpo pseudo-rígido, a qual simplifica drasticamente a determinação dos equilíbrios relativos e torna a questão da dinâmica matematicamente acessível. Com este tratamento, podemos relacionar ou comparar os resultados com aqueles estabelecidos na literatura, dentre eles: formato de equilíbrio de um fluido isolado em rotação, deformação de maré causada pela interação gravitacional e o torque de maré induzido no mesmo. Pela simplicidade do modelo pode-se ainda fazer uma análise qualitativa da dinâmica do sistema e obter estimativas sobre a velocidade com que se aproxima dos equilíbrios.

Palavras-chave: Força de maré, sistema dissipativo, corpo pseudo-rígido. 


\section{Abstract}

Ruiz, L. S. Dissipative Effects in Celestial Mechanics modeled by pseudo-rigid bodies. 2015. 81f. PhD thesis - Instituto de Matemática e Estatística, Universidade de São Paulo, São Paulo, 2015 .

The present work is devoted to model the interaction among celestial bodies, in a Newtonian regime, but considering the role played by the internal deformation and viscosity on the orbital motion and angular velocities of the components of the system. The work is mainly developed with an alternative approach to the pseudo-rigid body model, which simplifies the determination of the relative equilibria and allows precise conclusions about the dynamics. So, we are able to compare the results of this theory with those established in the literature, namely: the equilibrium shape of an isolated fluid in rotation, the tidal elongation induced by gravitational interaction and the tidal torque. Due to its simplicity, we can further perform a qualitative analysis of the dynamics of the system and estimate the velocity of attraction of the equilibrium states.

Keywords: Tidal force, dissipative system, pseudo-rigid body. 


\section{Sumário}

$\begin{array}{ll}\text { Lista de Símbolos } & \text { ix }\end{array}$

Lista de Figuras $\quad$ xi

1 Introdução $\quad 1$

1.1 O Problema da Força de Maré . . . . . . . . . . . . . . . . . . . . . . . 4

1.1.1 Corpo deformável auto-gravitante . . . . . . . . . . . . . . 5

1.1.2 Maré induzida por satélite . . . . . . . . . . . . . . . . . 8

2 Equações de movimento $\quad 9$

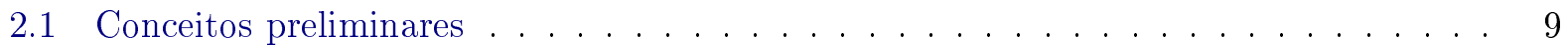

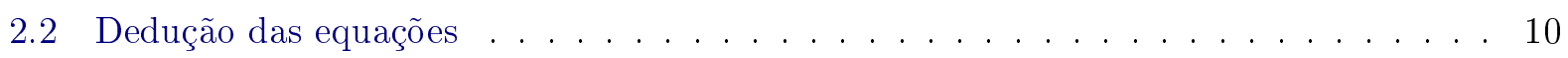

2.2 .1 Teorema de Noether . . . . . . . . . . . . . . . . . . . . . . 12

2.2.2 Equações de movimento em $\mathrm{SO}(3) \ldots \ldots \ldots \ldots \ldots$. . . . . . . . . . 13

2.2.3 Equações de movimento para o corpo girante . . . . . . . . . . . . . . . . . 14

2.2 .4 O caso incompressível . . . . . . . . . . . . . . . 16

2.2.5 Potenciais Elástico e Gravitacional . . . . . . . . . . . . . . . . . . 16

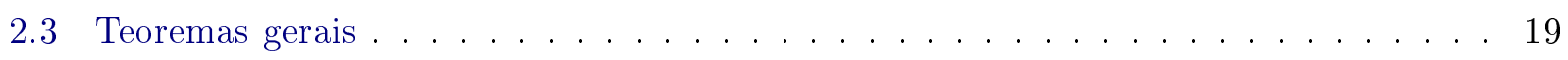

2.3.1 Princípio de LaSalle . . . . . . . . . . . . . . . . . . . . . . . . . . . 19

2.3 .2 Perturbação Singular . . . . . . . . . . . . . . . . . . . . . . . . 19

3 Equações de Movimento para Deformação Linear $\quad 23$

3.1 Corpos Pseudo-Rígidos . . . . . . . . . . . . . . . . . . . . . 23

3.1 .1 Equilíbrios para o modelo não linear . . . . . . . . . . . . . . . . . . 32

3.2 Corpo pseudo-rígido com pequenas deformações . . . . . . . . . . . . . . . . 37

3.3 Estabilidade linear . . . . . . . . . . . . . . . . . . . . . . . . 42

3.4 Problema de dois corpos . . . . . . . . . . . . . . . . . . . . 45

3.4.1 Número de Love dependente de frequência e o tempo de atraso . . . . . . . . 50

3.4.2 Equilíbrios para o problema de dois corpos . . . . . . . . . . . . . . . . 54

3.5 Determinação da constante efetiva $\tilde{\mu} \ldots \ldots \ldots \ldots \ldots$

3.6 Corpo Assimétrico e Ressonância Spin-Órbita . . . . . . . . . . . . . . . . . . 58

3.7 Problema de N Corpos . . . . . . . . . . . . . . . . . . . . . . . 59

$\begin{array}{ll}\text { Propostas de Estudo } & 61\end{array}$

$\begin{array}{ll}\text { A Potencial Gravitacional } & 65\end{array}$ 
$\begin{array}{ll}\text { B Aproximação do Potencial Gravitacional } & 67\end{array}$

B.1 Densidade homogênea . . . . . . . . . . . . . . . . . . . 67

B.2 Densidade não-homogênea . . . . . . . . . . . . . . . . . . 69

$\begin{array}{lll}\text { C Integral no problema de dois corpos } & 73\end{array}$

$\begin{array}{ll}\text { D Campo de forças no problema de } \mathrm{N} \text { corpos } & 75\end{array}$

$\begin{array}{ll}\text { E Número de Love } & 77\end{array}$

$\begin{array}{ll}\text { Referências Bibliográficas } & 79\end{array}$ 


\section{Lista de Símbolos}

\begin{tabular}{|c|c|}
\hline$\rho$ & Densidade de massa \\
\hline$\mu$ & Módulo elástico de cisalhamento \\
\hline$K$ & Módulo elástico volumétrico \\
\hline$\eta$ & Módulo de cisalhamento viscoso \\
\hline$\zeta$ & Módulo volumétrico viscoso \\
\hline $\mathcal{E}$ & Tensor de deformações \\
\hline$\sigma_{i k}$ & Tensor de tensões (stress tensor) \\
\hline$\tilde{\sigma}_{i k}$ & Tensor de tensões viscoso \\
\hline$\epsilon$ & Eliticidade ou achatamento \\
\hline$\tilde{\sigma}_{i k}$ & Tensor de tensões viscoso \\
\hline$u(t, x)$ & Deformação do corpo contínuo \\
\hline $\mathcal{B}$ & Configuração de repouso do corpo contínuo \\
\hline $\mathcal{C}\left(\mathcal{B}, \mathbb{R}^{3}\right)$ & Espaço das deformações $u(t, x)$ do corpo contínuo \\
\hline $\mathcal{C}_{0}\left(\mathcal{B}, \mathbb{R}^{3}\right)$ & Espaço das deformações $u(t, x)$, com $\operatorname{div} u(t, x)=0$ \\
\hline $\mathrm{M}(3)$ & Conjunto das matrizes quadradas de ordem 3 \\
\hline $\mathrm{GL}(3):=\{G \in \mathrm{M}(3): \operatorname{det}(G) \neq 0\}$ & Grupo linear geral \\
\hline $\operatorname{SL}(3):=\{G \in \mathrm{M}(3): \operatorname{det}(G)=1\}$ & Grupo linear especial \\
\hline $\mathrm{SO}(3):=\left\{Y \in \mathrm{SL}(3): Y^{-1}=Y^{T}\right\}$ & Grupo ortogonal especial \\
\hline $\operatorname{Sym}(3):=\left\{H \in \mathrm{M}(3): H^{T}=H\right\}$ & Conjunto das matrizes simétricas \\
\hline $\operatorname{skew}(3):=\left\{H \in \mathrm{M}(3): H^{T}=-H\right\}$ & Conjunto das matrizes antissimétricas \\
\hline $\operatorname{SSym}_{+}(3):=\{A \in \operatorname{Sym}(3) \cap \operatorname{SL}(3): A>0\}$ & Conjunto das deformações do corpo pseudo-rígido \\
\hline $\operatorname{ssym}(3):=\{B \in \operatorname{Sym}(3): \operatorname{Tr}(B)=0\}$ & Conjunto das deformações lineares infinitesimais \\
\hline$Y \in \mathrm{SO}(3)$ & Rotação do corpo \\
\hline$\Omega:=Y^{T} \dot{Y} \in$ skew $(3)$ & Velocidade angular no referencial do corpo \\
\hline$\Psi_{A}$ & Tensor de inércia do corpo pseudo-rígido \\
\hline$\Phi_{\varepsilon B}$ & Tensor de inércia truncado do corpo pseudo-rígido \\
\hline $\mathrm{I}_{\circ}$ & Momento de inércia da bola de repouso \\
\hline$h_{2}, k_{2}$ & Números de Love \\
\hline$\tilde{\mu}, \nu$ & $\begin{array}{l}\text { Constantes efetivas do corpo pseudo-rígido } \\
\text { com pequenas deformações }\end{array}$ \\
\hline$\tilde{M}:=M+m$ & Massa total do sistema de dois corpos \\
\hline$\tilde{m}:=M m /(M+m)$ & Massa reduzida do sistema de dois corpos \\
\hline
\end{tabular}




\section{Lista de Figuras}

1.1 Referencial Móvel Descontínuo. . . . . . . . . . . . . . . . . . . . . . 5

1.2 Coordenadas do problema de Kepler. . . . . . . . . . . . . . . . . . . . . 8

3.1 Operações envolvidas na decomposição singular. . . . . . . . . . . . . . . . . 25

3.2 Operações envolvidas na decomposição polar. . . . . . . . . . . . . . . . . . . . . . . . . . . .

3.3 Atraso no surgimento da maré devido à viscosidade. . . . . . . . . . . . . . . . . . . . . 52

3.4 Potência dissipada em função da frequência. . . . . . . . . . . . . . . . . . . . . . . . . . . . . . . . . .

3.5 Solução sem perda de energia. . . . . . . . . . . . . . . . . . . . . . . . 62 


\section{Capítulo 1}

\section{Introdução}

O estudo do problema de $N$ corpos em Mecânica Celeste é centrado em explorar as soluções da equação de Newton

$$
m_{i} \ddot{\overrightarrow{r_{i}}}=\sum_{j \neq i}^{N} \frac{G m_{i} m_{j}}{\left\|\vec{r}_{j}-\vec{r}_{i}\right\|^{3}}\left(\vec{r}_{j}-\vec{r}_{i}\right),
$$

sendo $m_{i}$ e $\vec{r}_{i}$ a massa e o vetor posição do $i$-ésimo corpo, respectivamente, e $G=6,674287 \times 10^{-11}$ $\mathrm{m}^{3} \mathrm{~kg}^{-1} \mathrm{~s}^{-2}$ a constante da Gravitação Universal. As observações mostram que as órbitas do Sistema Solar, por exemplo, podem ser analisadas a partir de uma superposição de problemas de dois corpos, regidos pela equação (1.1), com grande precisão. Os desvios observados deste modelo são detectados em uma escala de tempo, em geral, muito maior do que o período orbital de cada corpo.

No entanto, também observamos características comuns a boa parte dos corpos que não são genéricas no contexto das soluções da equação (1.1), como mostra a Tabela 1.1. Uma delas é o fato de quase todas as órbitas do sistema solar estarem contidas aproximadamente no plano da eclíptica (plano que contém a órbita da Terra em torno do Sol), ver a coluna inclinação orbital. Também observamos que as excentricidades orbitais são próximas de zero (desvios maiores são medidos em Mercúrio e Plutão). A terceira coluna mostra que o eixo de rotação de boa parte dos corpos é perpendicular ao seu plano orbital, notando que Vênus gira em uma direção retrógrada e que Urano e Plutão são exceções à regra.

Na Tabela 1.2 estão listados os maiores satélites naturais do Sistema Solar (com massa maior que $10^{21} \mathrm{~kg}$ ). Observe que todas as excentricidades orbitais são de fato próximas de zero. As inclinações das órbitas em relação à eclíptica, com exceção de Jápeto, Tritão e Caronte, são também próximas de zero. E, dado mais interessante, todos estes satélites executam um movimento síncrono em torno do corpo que orbitam, ou seja, possuem sempre a mesma face voltada para o corpo central.

Tabela 1.1: Dados orbitais dos corpos do Sistema Solar

\begin{tabular}{lccc}
\hline Corpo & Inclinação orbital (graus) & Excentricidade & Inclinação axial (graus) \\
\hline Mercúrio & 7,0 & 0,205 & 0,01 \\
Vênus & 3,4 & 0,007 & 177,4 \\
Terra & 0,0 & 0,017 & 23,4 \\
Lua & 5,1 & 0,055 & 6,7 \\
Marte & 1,9 & 0,094 & 25,2 \\
Júpiter & 1,3 & 0,049 & 3,1 \\
Saturno & 2,5 & 0,057 & 26,7 \\
Urano & 0,8 & 0,046 & 97,8 \\
Netuno & 1,8 & 0,011 & 28,3 \\
Plutão & 17,2 & 0,244 & 122,5 \\
\hline
\end{tabular}

Estes dados foram extraídos de (http://nssdc.gsfc.nasa.gov/planetary/planetfact.html). 
Tabela 1.2: Dados dos satélites do Sistema Solar

\begin{tabular}{llccc}
\hline Corpo & Satélite & Massa $\left(10^{20} \mathrm{~kg}\right)$ & Excentricidade & Inclinação orbital (graus) \\
\hline Terra & Lua & 734,8 & 0,055 & 5,1 \\
Júpiter & Io & 893,2 & 0,004 & 0,04 \\
Júpiter & Europa & 480,0 & 0,0101 & 0,47 \\
Júpiter & Ganimedes & 1481,9 & 0,0015 & 0,21 \\
Júpiter & Calisto & 1075,9 & 0,007 & 0,51 \\
Saturno & Dione & 11,0 & 0,0022 & 0,02 \\
Saturno & Reia & 23,1 & 0,0010 & 0,35 \\
Saturno & Titã & 1345,5 & 0,0292 & 0,33 \\
Saturno & Jápeto & 18,1 & 0,0283 & 14,72 \\
Urano & Ariel & 13,5 & 0,0034 & 0,31 \\
Urano & Umbriel & 11,7 & 0,0050 & 0,36 \\
Urano & Titânia & 35,2 & 0,0022 & 0,14 \\
Urano & Oberon & 30,1 & 0,0008 & 0,10 \\
Netuno & Tritão & 214, & 0,0000016 & 157,345 \\
Plutão & Caronte & 15,9 & 0,0 & 0,0 \\
\hline
\end{tabular}

* A inclinação de Caronte é em relação ao plano orbital de Plutão

Também damos destaque para o dados orbitais de Mercúrio. O tempo necessário para que ele dê uma volta completa em torno de seu eixo é $T_{D}=1407,5$ horas (período sinódico) e o tempo que gasta para completar uma translação em torno do Sol é $T_{A}=2111,4$ horas. A razão entre estes tempos é $T_{A} / T_{D}=1,49985 \approx 3 / 2$. Dizemos que Mercúrio está em ressonância spin-órbita $3: 2$ com o Sol.

Observamos assim que apesar de que em curtos intervalos de tempo o movimento seja bem descrito por (1.1) (mais um acoplamento com a velocidade angular de corpo rígido), efeitos observáveis em escalas de tempo bem maiores devem ser os responsáveis pela organização do sistema, dando origem a este padrão observado. Tais fenômenos são atribuídos às forças dissipativas, as quais atuam como perturbações do sistema original (1.1).

O objetivo deste trabalho é propor um modelo que descreva estes efeitos dentro do contexto da Mecânica Clássica, em um adequado regime de validade, mas sem atribuir restrições ao movimento, como impor movimento em um plano perpendicular ao eixo de rotação do corpo central. Apresentamos também um início da formulação do problema de $N$ corpos neste contexto.

Os assuntos abordados estão divididos da seguinte maneira:

1 - Introdução: Introduzimos os problemas de interesse, do corpo livre auto-gravitante e da força de maré sobre um corpo central, com citações de trabalhos pertinentes, fazendo uma breve exposição de cálculos e ideias bem difundidas sobre cada um.

2 - Equações de Movimento: Pretendemos abordar os temas da Introdução de forma geométrica, através de Lagrangianas e funções dissipação. Para isto expomos alguns resultados básicos desta formulação, como o método dos multiplicadores de Lagrange e o Teorema de Noether. Também obtemos, de forma heurística, equações de movimento idênticas (ou muito próximas) àquelas utilizadas em referências clássicas (por exemplo [Cha87], [Lov44] e [TT09]). Também apresentamos alguns resultados gerais da Teoria das Equações Diferenciais Ordinárias.

3 - Deformação Linear: Começamos com uma formulação precisa do problema do corpo deformável isolado, pseudo-rígido. Fazemos uma abordagem ligeiramente distinta da tradicional, obtendo informações sobre o movimento e equilíbrios. Em seguida, nos restringimos a deformações próximas à identidade, através de uma expansão em Taylor. Isto tornará as equações 
de movimento mais simples e intuitivas, permitindo obter as mesmas estimativas de achatamento nos polos de um corpo celeste, comparando com medidas, e o torque de maré tratado em [Mig79], com o tempo de atraso e os números de Love. Destaca-se que neste último não supomos rotação estacionária do corpo central, obtendo um aprimoramento do modelo anterior. Estimamos ainda a velocidade de atração dos equilíbrios.

A ferramenta matemática central utilizada neste trabalho será a equação de Euler-Lagrange com função dissipação, sendo esta última a responsável por introduzir as forças não conservativas do sistema mecânico, ver [Gol56]. Esta escolha facilitará a procura por integrais primeiras e a inserção de vínculos, os quais simplificarão drasticamente o problema.

Suponhamos que o espaço de configurações seja um aberto $U \subset \mathbb{R}^{n}$. Considere duas funções suaves, função Lagrangiana e função dissipação, $L, \mathcal{D}: U \times \mathbb{R}^{n} \longrightarrow \mathbb{R}$, respectivamente, $L(q, \dot{q}), \mathcal{D}(q, \dot{q})$. Aqui, como usual, identificamos o fibrado tangente $T U \cong U \times \mathbb{R}^{n}$, sendo que os pares $(q, \dot{q})$ denotam os pontos deste conjunto. Também denotamos por $\dot{q}$ a derivada de uma curva $q(t) \in U$ com relação ao seu parâmetro, denominado tempo.

Desta forma, as equações de movimento são calculadas da seguinte maneira

$$
\frac{d}{d t}\left(\frac{\partial L}{\partial \dot{q}}\right)-\frac{\partial L}{\partial q}=-\frac{\partial \mathcal{D}}{\partial \dot{q}}
$$

onde reservamos a notação $\partial / \partial \dot{q}$ e $\partial / \partial q$ para o gradiente da função na coordenada correspondente, segundo a métrica Euclidiana. Daqui em diante, caso não seja dito o contrário, estaremos seguindo esta convenção.

Lema 1.1. A primeira propriedade da equação (1.2) é ser invariante por mudanças de coordenadas.

Demonstração. Suponha que $Q(t)$ seja solução desta equação. Se $Q \rightarrow q(Q)$ é uma mudança de coordenadas, defina $L_{1}(Q, \dot{Q}):=L\left(q(Q), q^{\prime}(Q) \dot{Q}\right), \mathcal{D}_{1}(Q, \dot{Q}):=\mathcal{D}\left(q(Q), q^{\prime}(Q) \dot{Q}\right)$. Assim,

$$
\begin{gathered}
\partial_{Q} L_{1}=\partial_{q} L \circ q^{\prime}(Q)+\partial_{\dot{q}} L \circ d^{2} q(Q)(\dot{Q}, \cdot), \quad \partial_{\dot{Q}} L_{1}=\partial_{\dot{q}} L \circ q^{\prime}(Q), \quad \partial_{\dot{Q}} \mathcal{D}_{1}=\partial_{\dot{q}} \mathcal{D} \circ q^{\prime}(Q) \\
\Rightarrow \frac{d}{d t} \partial_{\dot{Q}} L_{1}=\frac{d}{d t}\left(\partial_{\dot{q}} L\right) \circ q^{\prime}(Q)+\partial_{\dot{q}} L \circ d^{2} q(Q)(\dot{Q}, \cdot),
\end{gathered}
$$

onde $d^{2} q(Q)$ é o Hessiano de $q(Q)$. Assim, usando o produto interno canônico,

$$
\frac{d}{d t}\left(\frac{\partial L_{1}}{\partial \dot{Q}}\right)-\frac{\partial L_{1}}{\partial Q}+\frac{\partial \mathcal{D}_{1}}{\partial \dot{Q}}=q^{\prime}(Q)^{T}\left[\frac{d}{d t}\left(\frac{\partial L}{\partial \dot{q}}\right)-\frac{\partial L}{\partial q}+\frac{\partial \mathcal{D}}{\partial \dot{q}}\right]
$$

Como $q(Q)$ é difeomorfismo, o primeiro termo é nulo se, e só se, o segundo também é. Ou seja, as equações (1.2) não dependem do sistema de coordenadas.

Observemos, através de um exemplo, que em geral não é possível encontrar uma Lagrangiana, independente do tempo, que gere as equações de movimento de um sistema mecânico dissipativo. Considere o oscilador harmônico amortecido

$$
\ddot{x}+\nu \dot{x}+\mu x=0 .
$$

Afirmação 1. Nẫo existe $L(x, \dot{x})$ suave, definida em aberto contendo a origem, tal que (1.4) seja a equação de Euler-Lagrange, na forma usual, gerada pela mesma.

Demonstração. Como se sabe, o equilíbrio $(x, \dot{x})=(0,0)$ é assintoticamente estável, $\forall \mu, \nu>0$. Suponha que exista tal função. Segue diretamente que

$$
H(x, \dot{x}):=\dot{x} \frac{\partial L}{\partial \dot{x}}-L(x, \dot{x})
$$


é constante ao longo das soluções de (1.4). Portanto, em torno da origem,

$$
L(x, y)=y \frac{\partial L}{\partial y}-H_{0},
$$

com $H_{0}$ constante. Logo, $\forall y \neq 0$,

$$
\frac{\partial}{\partial y}\left(\frac{L+H_{0}}{y}\right)=\frac{1}{y^{2}}\left(y \frac{\partial L}{\partial y}(x, y)-L(x, y)-H_{0}\right)=0 .
$$

Assim, existem funções suaves $C_{1}(x), C_{2}(x)$ tais que

$$
L(x, y)+H_{0}= \begin{cases}C_{1}(x) y, & y>0 \\ C_{2}(x) y, & y<0 .\end{cases}
$$

Note que pela suavidade das funções, para todo $x$ próximo de 0 ,

$$
C_{1}(x)=\lim _{y \rightarrow 0+} \frac{\partial L}{\partial y}(x, y)=\lim _{y \rightarrow 0-} \frac{\partial L}{\partial y}(x, y)=C_{2}(x)=: C(x) .
$$

Assim, a equação de Euler-Lagrange gerada é

$$
\frac{d}{d t}(C(x))-C^{\prime}(x) \dot{x}=0,
$$

a qual claramente não é equivalente a (1.4). Daí vem a necessidade do termo dissipativo em (1.2).

Em geral, o fluxo de um sistema dissipativo não preserva o volume no espaço de fase, fato que o impede de ser um sistema Hamiltoniano.

Notamos que $L(x, \dot{x}, t):=\dot{x}^{2} / 2-\mu x^{2} / 2$ e $\mathcal{D}:=\nu \dot{x}^{2} / 2$ geram a equação de movimento (1.4) através de (1.2). Assim como $L(x, \dot{x}, t):=e^{\nu t}\left(\dot{x}^{2}-\mu x^{2}\right)$ e $\mathcal{D}=0$, mas neste caso $L$ depende do tempo.

Na próxima seção, expomos uma abordagem usual da força de maré sobre corpos celestes, fazendo um esboço da dedução de suas fórmulas, as quais compararemos com as obtidas em nosso modelo, no Capítulo 3. O objetivo de expor este método é para ressaltar a independência do tratamento que estamos propondo.

\subsection{O Problema da Força de Maré}

O fenômeno da força de maré é observado como uma perturbação do movimento do sistema isolado de dois corpos interagindo gravitacionalmente.

Em primeira aproximação, consideramos os dois corpos como pontuais, e que o movimento é descrito pela mecânica Newtoniana de partículas (1.1). Tal movimento é completamente entendido, no sentido que conhecemos explicitamente as trajetórias dos corpos (porém determinar o tempo de percurso é tarefa difícil).

Uma segunda aproximação, mais elaborada, é considerar a massa de um dos corpos muito superior à do segundo (planeta e satélite, estrela e planeta), e tentar entender qual é o efeito da forma geométrica do satélite sobre seu próprio movimento orbital. Apesar de não conhecermos as soluções explícitas deste problema geral, ele também foi explorado por diversos autores, tanto do ponto de vista dos equilíbrios (problema de Roche), quanto do ponto de vista dinâmico [KWM91] (explorando a formulação geométrica do problema).

No problema de Roche, trata-se o satélite como um fluido, por isso se restringe à análise dos equilíbrios. No segundo caso, considera-se o satélite como um corpo rígido, e o planeta como uma esfera de massa muito maior, de forma que seu campo seja dado pela lei da Gravitação de Newton, centrada na origem. Mas, ainda é possível analisar a evolução dinâmica do sistema fazendo hipóteses 
restritivas sobre o satélite, supondo este deformável, mas não de forma arbitrária. Um exemplo é supor que o satélite se comporta como um corpo pseudo-rígido [KVG $\left.{ }^{+} 12\right]$.

Uma terceira aproximação é considerar o satélite como pontual e o planeta como corpo contínuo, deformável, cujo centro de massa é fixo na origem. O movimento de rotação do planeta em torno de si próprio e a rotação do satélite ao redor do planeta induzem deformações neste último, as quais influenciarão em sua própria rotação e no movimento do satélite. Temos, assim, um acoplamento.

Supondo-se que o planeta é um fluido viscoso, são propostos alguns modelos que prevêem a força adicional à Newtoniana exercida sobre o satélite. Uma força amplamente usada é devida a Mignard [Mig79]. Para uma revisão histórica, consulte [EW09].

\subsubsection{Corpo deformável auto-gravitante}

Analisemos a abordagem usual para o problema de um corpo contínuo, deformável, isolado no espaço. É natural pensarmos numa pequena variação do problema do corpo rígido, imaginando este corpo como um sólido, que pouco se deforma. Desta forma, nos perguntamos qual seria um boa escolha de referencial que acompanha o mesmo. Assim nos deparamos com uma questão delicada. Um tal referencial dependeria do movimento da matéria que constitui o corpo e talvez de seu formato.

Lembremos como procedemos para o corpo rígido. Uma das maneiras de se determinar o referencial móvel é através do tensor de inércia, o qual depende do formato do corpo. Para cada instante de tempo, ele é representado por uma matriz simétrica, positiva definida, cujos coeficientes são funções suaves. Seus autovalores são constantes no tempo e, caso sejam distintos entre si, tomamos os autovetores correspondentes como base (ordenada de acordo com a magnitude dos autovalores) do referencial móvel (suave).

Quando permitimos que o corpo se deforme, para cada instante de tempo podemos calcular seu tensor de inércia e tomar da mesma forma este referencial. Porém, caso a deformação (suave) se dê próxima a um formato esférico, podemos ter o movimento esboçado na Figura 1.1. Adotamos a convenção de que $\vec{e}_{1}$ e $\vec{e}_{2}$ estão nas direções dos eixos de menor e maior inércia, respectivamente, de forma que $\left(\vec{e}_{1}, \vec{e}_{2}\right)$ seja base positiva.

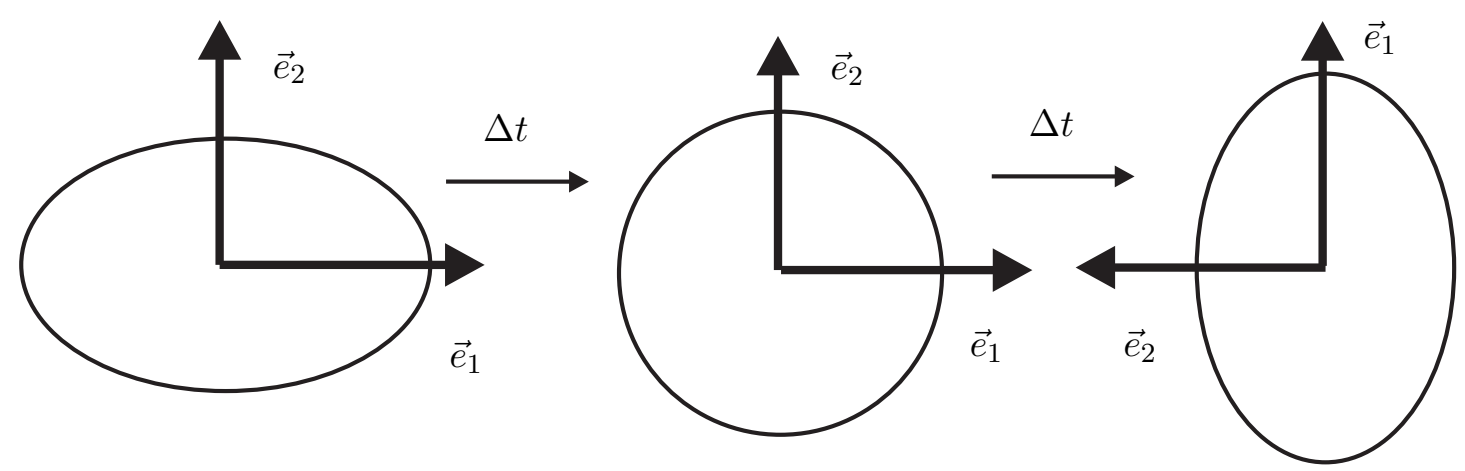

Figura 1.1: Referencial Móvel Descontínuo.

Claramente esta situação é indesejada, já que teremos um referencial descontínuo e necessitamos calcular derivadas das componentes destes vetores para escrever as equações de movimento. Tal abordagem é atribuída a alguns trabalhos clássicos sobre o assunto, como [Dar08]. Tentaremos evitar este tipo de dificuldade, com outra escolha de referencial móvel.

Seguindo [Fit12], por exemplo, considere o corpo como sendo um fluido com uma densidade uniforme, ocupando uma região $\mathcal{B} \subset \mathbb{R}^{3}$. O potencial gravitacional gerado por ele em um ponto $x \in \mathbb{R}^{3}$ é

$$
V(x)=-G \int_{\mathcal{B}} \frac{\rho(y)}{\|x-y\|} d y .
$$


Tome coordenadas esféricas e suponha que (no equilíbrio relativo) o corpo tenha o formato de um elipsoide de revolução em torno do eixo $z$. Fazendo a densidade ser constante no corpo e nula no exterior, podemos trocar a integral anterior por uma integral em $\mathbb{R}^{3}$, envolvendo a densidade que depende apenas de $r$ e $\theta$.

$$
V(r, \theta)=-G \int_{0}^{\infty} \int_{0}^{\pi} \int_{0}^{2 \pi} \frac{\rho\left(r^{\prime}, \theta^{\prime}\right)}{\|x-y\|} r^{\prime 2} \operatorname{sen} \theta^{\prime} d \phi^{\prime} d \theta^{\prime} d r^{\prime}
$$

sendo $x=(r \operatorname{sen} \theta \cos \phi, r \operatorname{sen} \theta \operatorname{sen} \phi, r \cos \theta), y=\left(r^{\prime} \operatorname{sen} \theta^{\prime} \cos \phi^{\prime}, r^{\prime} \operatorname{sen} \theta^{\prime} \operatorname{sen} \phi^{\prime}, r^{\prime} \cos \theta^{\prime}\right)$.

Note que $V$ independe de $\phi$ devido à simetria do corpo. Fato bem conhecido é que podemos tomar a seguinte expansão em polinômios de Legendre de $\cos \theta$,

$$
\int_{0}^{2 \pi} \frac{1}{\|x-y\|} d \phi^{\prime}=2 \pi \frac{1}{r} \sum_{n=0}^{\infty}\left(\frac{r^{\prime}}{r}\right)^{n} P_{n}\left(\cos \theta^{\prime}\right) P_{n}(\cos \theta), \quad\|y\| \geq\|x\| .
$$

Por integração, (1.5) fica na forma:

$$
V(r, \theta)=\sum_{n=0}^{\infty} J_{n} \frac{P_{n}(\cos \theta)}{r^{n+1}} .
$$

Para determinarmos $J_{n}$, devemos especificar a densidade. Para um elipsoide de raio médio $R$, propõe-se que sua superfície seja dada pela equação:

$$
r=r_{\epsilon}(\theta)=R\left(1-\frac{2}{3} \epsilon P_{2}(\cos \theta)\right)=R\left(\frac{\epsilon}{3}+1-\epsilon \cos ^{2} \theta\right),
$$

onde $\epsilon>0$ é chamada a eliticidade do elipsoide (ver (3.32)) e $R$ um raio médio. A densidade tem a forma

$$
\rho(r, \theta)=\left\{\begin{array}{cc}
\rho_{0}, & r \leq r_{\epsilon}(\theta) \\
0, & r>r_{\epsilon}(\theta)
\end{array}\right.
$$

Desta forma,

$$
J_{n}=-G \rho_{0} \int_{0}^{\pi} P_{n}\left(\cos \theta^{\prime}\right) \int_{0}^{r_{\epsilon}\left(\theta^{\prime}\right)} r^{\prime 2+n} d r^{\prime} \operatorname{sen} \theta^{\prime} d \theta^{\prime}=-\frac{G \rho_{0}}{3+n} \int_{0}^{\pi} P_{n}\left(\cos \theta^{\prime}\right) r_{\epsilon}^{3+n}\left(\theta^{\prime}\right) \operatorname{sen} \theta^{\prime} d \theta^{\prime} .
$$

Assumindo que $\epsilon$ é um parâmetro pequeno, aproximamos $r_{\epsilon}^{3+n}\left(\theta^{\prime}\right)$ pelo seu polinômio de Taylor de primeira ordem em $\epsilon$ :

$$
r_{\epsilon}^{3+n}(\theta)=P_{0}\left(\cos \theta^{\prime}\right)-\frac{2}{3}(3+n) \epsilon P_{2}\left(\cos \theta^{\prime}\right)+\mathcal{O}\left(\epsilon^{2}\right) .
$$

Pela ortogonalidade dos polinômios de Legendre, vemos que em primeira ordem em $\epsilon$ os únicos coeficientes não nulos são $J_{0}$ e $J_{2}$. Procedendo com o cálculo, obtemos:

$$
V(r, \theta)=-\frac{G M}{r}+\frac{2}{5} \frac{G M R^{2}}{r^{3}} \epsilon P_{2}(\cos \theta)+\mathcal{O}\left(\epsilon^{2}\right),
$$

onde $M$ é a massa do corpo.

O potencial na superfície do elipsoide é calculado fazendo $r=r_{\epsilon}(\theta)$. Expandindo novamente a expressão resultante até ordem $\epsilon$, obtemos:

$$
V_{g}(\theta)=V\left(r_{\epsilon}(\theta), \theta\right)=-\frac{G M}{R}\left(1+\frac{4}{15} \epsilon P_{2}(\cos \theta)\right)+\mathcal{O}\left(\epsilon^{2}\right) .
$$

Por outro lado, podemos estimar o efeito de deformação sobre um fluido em formato esférico sujeito a uma rotação de velocidade angular uniforme $\omega$ em torno do eixo $z$. Tomando um referencial 
que se mova deste modo, em coordenadas esféricas, encontramos as forças inerciais resultantes.

Para um ponto (fixo) sobre a superfície, temos somente a aceleração centrífuga:

$$
a_{c}(\theta)=r \omega^{2} \operatorname{sen} \theta(\operatorname{sen} \theta, \cos \theta, 0) .
$$

Note que $a_{c}=-\nabla V_{c}(r, \theta)$, sendo

$$
V_{c}(r, \theta)=-\frac{\omega^{2} r^{2}}{3}\left(1-P_{2}(\cos \theta)\right)
$$

Pelo que vimos, a força gravitacional exercida na superfície do corpo é dada pelo (negativo do) gradiente de (1.6). Sendo assim, a força resultante na superfície é dada por:

$$
f_{\text {Res }}=-\nabla\left(V(r, \theta)+V_{c}(r, \theta)\right) .
$$

A condição de equilíbrio impõe que na superfície este gradiente seja nulo. Assim, encontramos que

$$
V\left(r_{\epsilon}(\theta), \theta\right)+V_{c}\left(r_{\epsilon}(\theta), \theta\right)=c, \quad \forall \theta
$$

sendo $c$ constante.

Assim, em primeira ordem em $\epsilon$, obtemos:

$$
-\frac{G M}{R}\left(1+\frac{4}{15} \epsilon P_{2}(\cos \theta)\right)-\frac{\omega^{2} R^{2}}{3}\left(1-P_{2}(\cos \theta)\right) \approx c .
$$

Pela independência dos polinômios de Legendre, concluímos:

$$
\epsilon=\frac{5}{4} \frac{\omega^{2} R^{3}}{G M}
$$

Note que esta estimativa é válida somente para baixas velocidades angulares.

O caso mais simples de corpo não-homogêneo seria um composto por duas camadas, cada uma com densidade homogênea, por analogia ao núcleo e manto terrestres. A densidade neste caso é

$$
\rho(r, \theta)=\left\{\begin{aligned}
\rho_{1}, & r & \leq r_{1 \epsilon}(\theta), \\
\rho_{2}, & r_{1 \epsilon}(\theta) & <r<r_{2 \epsilon}(\theta), \\
0, & r & >r_{2 \epsilon}(\theta) .
\end{aligned}\right.
$$

sendo

$$
r_{j \epsilon}(\theta)=R_{j}\left(1-\frac{2}{3} \epsilon P_{2}(\cos \theta)\right), \quad j=1,2 .
$$

De maneira análoga ao caso anterior, obtemos:

$$
J_{n}=\frac{2 \pi G}{3+n} \int_{0}^{\pi} P_{n}(\cos \theta)\left(\left(\rho_{1}-\rho_{2}\right) r_{1 \epsilon}^{3+n}(\theta)+\rho_{2} r_{2 \epsilon}^{3+n}(\theta)\right) \operatorname{sen} \theta d \theta .
$$

Novamente, após tomar termos até ordem um em $\epsilon$, concluímos que:

$$
J_{0}=-\frac{4 \pi G}{3}\left(\left(\rho_{1}-\rho_{2}\right) R_{1}^{3}+\rho_{2} R_{2}^{3}\right), \quad J_{2}=\frac{8 \pi G \epsilon}{15}\left(\left(\rho_{1}-\rho_{2}\right) R_{1}^{5}+\rho_{2} R_{2}^{5}\right) .
$$

Por fim, igualando novamente os potenciais na superficie, encontramos que a eliticidade fica:

$$
\epsilon=\frac{15 \Omega^{2} R_{2}^{5}}{8 \pi G}\left(2 \rho_{2} R_{2}^{5}+\left(\rho_{1}-\rho_{2}\right)\left(5 R_{2}^{2}-3 R_{1}^{2}\right) R_{1}^{3}\right)^{-1}
$$




\subsubsection{Maré induzida por satélite}

A expressão (1.6) também é facilmente aplicada ao problema de um corpo central (planeta) e um corpo orbitante (satélite), de massa desprezível em relação ao primeiro. Aqui, desprezaremos a rotação do planeta em torno de seu eixo, mas consideraremos o movimento do seu centro de massa. Assumimos que os dois corpos giram em torno do centro de massa do sistema, em movimento circular de velocidade angular $\omega$. Considere que cada elemento de massa do planeta gira com esta velocidade angular, a uma distância $\ell$ deste centro.

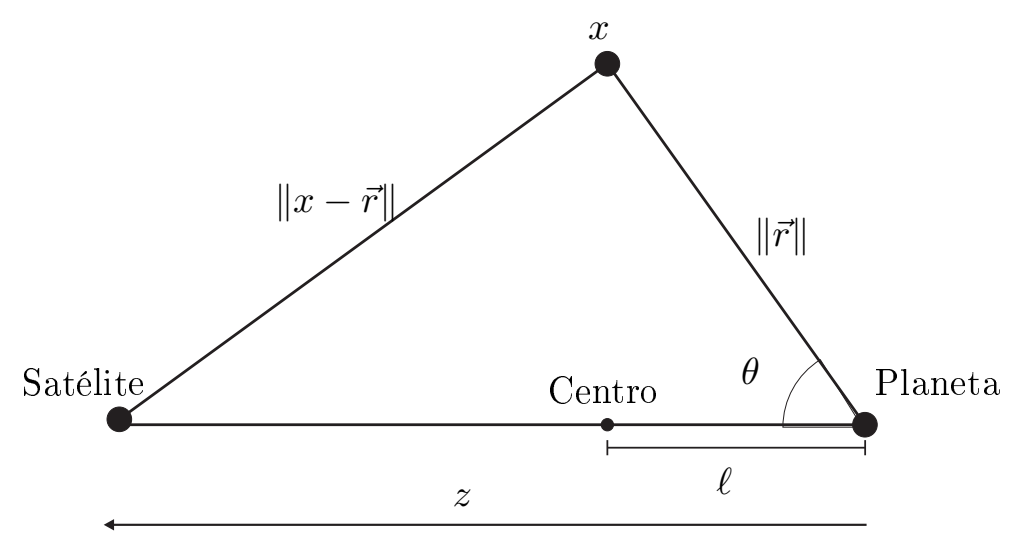

Figura 1.2: Coordenadas do problema de Kepler.

A aceleração centrífuga sobre cada elemento é dada por $a_{c}=-\omega^{2} \ell \hat{e}_{z}$, tomando como eixo $z$ aquele que liga os dois corpos e $\hat{e}_{z}$ o versor unitário nesta direção. Assim, $a_{c}=-\nabla V_{\text {cent }}$, onde:

$$
V_{\text {cent }}(z)=\omega^{2} \ell z .
$$

Sendo $m$ e $\vec{r}$ a massa e a posição do satélite respectivamente, e tomando coordenadas esféricas $x=(r \operatorname{sen} \theta \cos \phi, r \operatorname{sen} \theta \operatorname{sen} \phi, r \cos \theta)$, o potencial gravitacional gerado é:

$$
V_{\text {sat }}(x)=-\frac{G m}{\|x-\vec{r}\|}=-\frac{G m}{\|\vec{r}\|} \sum_{n=0}^{\infty}\left(\frac{r}{\|\vec{r}\|}\right)^{n} P_{n}(\cos \theta) .
$$

Assumimos que o raio do planeta é pequeno em relação à distância ao satélite. Como estaremos interessados novamente no equilíbrio das forças na superfície do planeta, desprezaremos todos os termos com $n \geq 3$ da série acima. Procedendo com um raciocínio análogo ao da seção anterior, impondo força resultante nula sobre a superfície do planeta, obtém-se a seguinte eliticidade induzida pela força de maré:

$$
\epsilon=-\frac{15}{4} \frac{m}{M}\left(\frac{R}{r_{0}}\right)^{3},
$$

onde $r_{0}$ é a distância entre os corpos no equilíbrio. 


\section{Capítulo 2}

\section{Equações de movimento}

Nosso objetivo é tratar a evolução temporal de um sistema mecânico (contínuo) em rotação no espaço e algumas variações deste problema. Uma tentativa de modelagem completa naturalmente nos leva a equações de movimento muito complexas. Portanto, almejando simplificar o modelo reduzindo seus graus de liberdade, escolhemos usar o formalismo Lagrangiano para descrevê-lo. Além disso, gostaríamos de entender a influência da viscosidade, a qual induz perda de energia nestes movimentos. Como observado na Introdução, mesmo sistemas dissipativos simples não se expressam através de equações de Euler-Lagrange de uma Lagrangiana independente do tempo. Desta forma, tentaremos expressar a viscosidade através de uma função dissipação.

Esta abordagem é apresentada na próxima seção, seguindo as ideias da referência [BL87].

Uma referência suficiente para uma revisão de mecânica Lagrangiana se encontra em [Arn89].

\subsection{Conceitos preliminares}

O corpo rígido consiste em um sistema mecânico contínuo que possui como vínculos (holonômi$\cos )$ :

$$
\left\|r\left(x_{1}, t\right)-r\left(x_{2}, t\right)\right\|=r_{12}, \quad \forall t \geq 0,
$$

sendo $x_{1}, x_{2}$ as coordenadas de pontos do corpo na posição inicial, e $r\left(x_{1}, t\right), r\left(x_{2}, t\right)$ suas posições após um tempo $t$ ( $r_{12}$ é constante no tempo).

Neste caso mostra-se [Arn89] que os graus de liberdade são determinados pelas seguintes coordenadas (independentes): $(Y, y) \in \mathrm{SO}(3) \times \mathbb{R}^{3}$. Tais coordenadas representam, respectivamente, a rotação de um referencial fixo ao corpo e o deslocamento de sua origem (o qual move-se "rigidamente" com o corpo), em relação ao referencial inercial adotado.

Para descrever um corpo "aproximadamente rígido" ou sólido, adicionamos a essas coordenadas uma coordenada "contínua", $u$, associada às deformações sofridas pelas componentes do corpo, no referencial móvel.

A manipulação desta coordenada será formal, sem estabelecer um espaço adequado ao qual pertença. Assumamos inicialmente que $u(\cdot, t) \in \mathcal{C}\left(\mathcal{B}, \mathbb{R}^{3}\right)$, um espaço vetorial ou uma vizinhança do vetor nulo de um espaço vetorial [BL91], sendo $\mathcal{B} \subset \mathbb{R}^{3}$ o conjunto que delimita a extensão espacial do corpo na posição inicial, ou relaxada, sem deformações.

As coordenadas $(Y, y)$ determinam um referencial no qual o corpo está em repouso. Qualquer vetor $v$ nesse referencial é representado no referencial inercial como $V$ através da equação:

$$
V(t)=Y(t) v(t)+y(t)
$$

Desde que $Y(t) \in \mathrm{SO}(3)$ é suave, $\forall t, \dot{Y}(t)=Y(t) \Omega(t)$, sendo

$$
\Omega(t)=\left(\begin{array}{ccc}
0 & -\omega_{3} & \omega_{2} \\
\omega_{3} & 0 & -\omega_{1} \\
-\omega_{2} & \omega_{1} & 0
\end{array}\right)
$$


uma matriz antissimétrica. O vetor $\omega(t)=\left(\omega_{1}, \omega_{2}, \omega_{3}\right)$ é denominado velocidade angular do corpo descrita no referencial móvel.

\subsection{Dedução das equações}

Uma maneira de deduzir as equações de movimento seria através de coordenadas angulares para $\mathrm{SO}(3)$, como os ângulos de Euler. Como se sabe, tal parametrização possui singularidades. Para evitar esta abordagem, podemos utilizar o conhecido Teorema dos Multiplicadores de Lagrange. Podemos adaptá-lo facilmente ao nosso contexto, com a função dissipação. Por isso tentamos apresentar uma discussão completa nesta seção.

Tome inicialmente $M$, uma variedade diferenciável de dimensão $n$.

Fixando a notação, associado a $M$, temos seu fibrado tangente

$$
T M:=\bigcup_{x \in M}\{x\} \times T_{x} M .
$$

Sabe-se que este também é uma variedade com o atlas: $\mathcal{A}_{T M}=\{d \varphi: \varphi \in \mathcal{A}\}$, sendo $\mathcal{A}$ um atlas de $M$. Dado $\varphi: U \subset M \longrightarrow \tilde{U} \subset \mathbb{R}^{n}$, denotamos por $d \varphi: T U \longrightarrow \tilde{U} \times \mathbb{R}^{n}, d \varphi(x, v):=\left(\varphi(x), d \varphi_{x} v\right)$ sua diferencial, sendo $T U=\bigcup_{x \in U}\{x\} \times T_{x} M$.

Sejam $L, D: T M \longrightarrow \mathbb{R}$ funções suaves, a Lagrangiana e a função dissipação do sistema. Tome $x \in M$ e uma carta $\varphi$ tal que $x$ esteja em seu domínio. Denote por $\mathcal{L}:=L \circ d \varphi^{-1}: \tilde{U} \times \mathbb{R}^{n} \longrightarrow \mathbb{R}$ e $\mathcal{D}:=D \circ d \varphi^{-1}: \tilde{U} \times \mathbb{R}^{n} \longrightarrow \mathbb{R}$ suas representações nesta carta.

Definição 2.1. Diremos que uma curva suave, $\gamma: I \subset \mathbb{R} \longrightarrow M$, com $0 \in I, \gamma(0)=x$, satisfaz à equação de Euler-Lagrange com dissipação em $x$ (em relação a L e D) se existe $\varphi: x \in U \longrightarrow \tilde{U} \subset \mathbb{R}^{n}$ tal que

$$
\frac{d}{d t}\left(\partial_{\dot{Q}} \mathcal{L}\left(d \varphi\left(\gamma(t), \gamma^{\prime}(t)\right)\right)\right)-\partial_{Q} \mathcal{L}\left(d \varphi\left(\gamma(t), \gamma^{\prime}(t)\right)\right)+\partial_{\dot{Q}} \mathcal{D}\left(d \varphi\left(\gamma(t), \gamma^{\prime}(t)\right)\right)=0, \quad \forall t .
$$

Note que $\mathcal{L}, \mathcal{D} \in \mathcal{C}^{\infty}\left(\tilde{U} \times \mathbb{R}^{n}\right)$. Assim, as derivadas parciais acima denotam a diferencial com respeito à correspondente coordenada. Se $(\alpha(t), \beta(t)) \in \tilde{U} \times \mathbb{R}^{n}$,

$$
\partial_{\dot{Q}} \mathcal{L}(\alpha(t), \beta(t)):=d(\mathcal{L}(\alpha(t), \cdot))_{\beta(t)} \in\left(\mathbb{R}^{n}\right)^{*}, \quad \partial_{Q} \mathcal{L}(\alpha(t), \beta(t)):=d(\mathcal{L}(\cdot, \beta(t)))_{\alpha(t)} \in\left(\mathbb{R}^{n}\right)^{*},
$$

e da mesma forma para $\mathcal{D}$.

Portanto, a equação estabelece que, para cada $t$ no domínio descrito, este funcional (sobre $\mathbb{R}^{n}$ ) é identicamente nulo.

Observe que a equação (1.3) garante que a definição 2.1 não depende da carta que tomemos.

O Teorema dos Multiplicadores de Lagrange pode ser enunciado da seguinte forma.

Teorema 2.2. Seja $M$ uma variedade diferenciável e $M_{0} \subset M$ uma subvariedade de dimensão $k \leq n$. Suponha que $L, D \in \mathcal{C}^{\infty}(T M)$. Sendo assim, se $\gamma: I \longrightarrow M_{0}$ satisfaz à equação de EulerLagrange com dissipação em $x \in M_{0}$ (em relação a $\left.L\right|_{T M_{0}},\left.D\right|_{T M_{0}}$ ), então existe carta $\varphi: x \in U \subset$ $M \longrightarrow \tilde{U}$ tal que

$$
\left\{\frac{d}{d t}\left(\partial_{\dot{Q}} \mathcal{L}\left(d \varphi\left(\gamma(t), \gamma^{\prime}(t)\right)\right)\right)-\partial_{Q} \mathcal{L}\left(d \varphi\left(\gamma(t), \gamma^{\prime}(t)\right)\right)+\partial_{\dot{Q}} \mathcal{D}\left(d \varphi\left(\gamma(t), \gamma^{\prime}(t)\right)\right)\right\}(0, v)=0 \quad \forall v \in \mathbb{R}^{k}
$$

onde, $(0, v) \in \mathbb{R}^{n-k} \times \mathbb{R}^{k} \equiv \mathbb{R}^{n}$ é um vetor cujas $n-k$ primeiras componentes são nulas, e as demais são as componentes de $v$.

Demonstração. Como $M_{0}$ é subvariedade de $M$, existe uma carta $\varphi$, como mencionada no enunciado tal que $\varphi\left(U \cap M_{0}\right)=\{0\}_{n-k} \times \tilde{U}_{0} \subset\{0\}_{n-k} \times \mathbb{R}^{k}$, onde $\operatorname{dim} M=n \geq \operatorname{dim} M_{0}=k$ (uma carta de 
subvariedade de $M_{0}$ em $x$ ). Defina, $P_{2}: \mathbb{R}^{n} \longrightarrow \mathbb{R}^{k}$ e $I_{2}: \mathbb{R}^{k} \longrightarrow \mathbb{R}^{n}$, por:

$$
P_{2}(a, b):=b, \quad I_{2}(b):=(0, b) .
$$

Defina também a inclusão, $i: M_{0} \longrightarrow M, i(x)=x, \forall x \in M_{0}$. Sendo assim, é fácil verificar que $\varphi_{0}:=P_{2} \circ \varphi \circ i: U \cap M_{0} \longrightarrow \tilde{U}_{0}$, é carta de $M_{0}$. Além disso, $\forall y \in U \cap M_{0}, \varphi \circ i(y)=(0, u) \in$ $\{0\}_{n-k} \times \tilde{U}_{0}$. Mas, $\varphi_{0}(y)=P_{2}(0, u)=u \Rightarrow \varphi \circ i=I_{2} \circ \varphi_{0}$.

Logo, pela hipótese,

$$
\frac{d}{d t}\left(\partial_{\dot{Q}} \mathcal{L}_{0}\left(d \varphi_{0}\left(\gamma(t), \gamma^{\prime}(t)\right)\right)\right)-\partial_{Q} \mathcal{L}_{0}\left(d \varphi_{0}\left(\gamma(t), \gamma^{\prime}(t)\right)\right)+\partial_{\dot{Q}} \mathcal{D}_{0}\left(d \varphi_{0}\left(\gamma(t), \gamma^{\prime}(t)\right)\right)=0,
$$

onde, $\mathcal{L}_{0}:=\left.L\right|_{T M_{0}} \circ d \varphi_{0}^{-1}=L \circ d i \circ d \varphi_{0}^{-1}=L \circ d\left(i \circ \varphi_{0}^{-1}\right)=L \circ d\left(\varphi^{-1} \circ I_{2}\right)=\mathcal{L} \circ I_{2}$. Sendo $\mathcal{L}:=L \circ d \varphi^{-1}$. Analogamente $\mathcal{D}_{0}=\mathcal{D} \circ I_{2}, \operatorname{com} \mathcal{D}:=D \circ d \varphi^{-1}$.

Desta forma,

$$
\begin{gathered}
\left.\partial_{\dot{Q}} \mathcal{L}\left(d \varphi\left(\gamma(t), \gamma^{\prime}(t)\right)\right) \circ I_{2}=\partial_{\dot{Q}} \mathcal{L}\left(I_{2} \circ \varphi_{0}(\gamma(t)), I_{2} \circ d\left(\varphi_{0}\right)_{\gamma(t)} \gamma^{\prime}(t)\right)\right) \circ I_{2} \\
\left.=\partial_{\dot{Q}}\left(\mathcal{L} \circ I_{2}\right)\left(\varphi_{0}(\gamma(t)), d\left(\varphi_{0}\right)_{\gamma(t)} \gamma^{\prime}(t)\right)\right)=\partial_{\dot{Q}} \mathcal{L}_{0}\left(d \varphi_{0}\left(\gamma(t), \gamma^{\prime}(t)\right)\right) . \\
\partial_{\dot{Q}} \mathcal{D}\left(d \varphi\left(\gamma(t), \gamma^{\prime}(t)\right)\right) \circ I_{2}=\partial_{\dot{Q}} \mathcal{D}_{0}\left(d \varphi_{0}\left(\gamma(t), \gamma^{\prime}(t)\right)\right) . \\
\left.\partial_{Q} \mathcal{L}\left(d \varphi\left(\gamma(t), \gamma^{\prime}(t)\right)\right) \circ I_{2}=\partial_{Q} \mathcal{L}\left(I_{2} \circ \varphi_{0}(\gamma(t)), I_{2} \circ d\left(\varphi_{0}\right)_{\gamma(t)} \gamma^{\prime}(t)\right)\right) \circ I_{2} \\
\left.=\partial_{Q}\left(\mathcal{L} \circ I_{2}\right)\left(\varphi_{0}(\gamma(t)), d\left(\varphi_{0}\right)_{\gamma(t)} \gamma^{\prime}(t)\right)\right)=\partial_{Q} \mathcal{L}_{0}\left(d \varphi_{0}\left(\gamma(t), \gamma^{\prime}(t)\right)\right) .
\end{gathered}
$$

De acordo com (2.3), segue que

$$
\begin{aligned}
\frac{d}{d t}\left(\partial_{\dot{Q}} \mathcal{L}_{0}\left(d \varphi_{0}\left(\gamma(t), \gamma^{\prime}(t)\right)\right)\right)-\partial_{Q} \mathcal{L}_{0}\left(d \varphi_{0}\left(\gamma(t), \gamma^{\prime}(t)\right)\right)+\partial_{\dot{Q}} \mathcal{D}_{0}\left(d \varphi_{0}\left(\gamma(t), \gamma^{\prime}(t)\right)\right) \\
\quad=\left(\frac{d}{d t}\left(\frac{\partial \mathcal{L}}{\partial \dot{Q}}\left(d \varphi\left(\gamma(t), \gamma^{\prime}(t)\right)\right)\right)-\frac{\partial \mathcal{L}}{\partial Q}(d \varphi(\gamma(t), \gamma(t)))+\frac{\partial \mathcal{D}}{\partial \dot{Q}}\left(d \varphi\left(\gamma(t), \gamma^{\prime}(t)\right)\right)\right) \circ I_{2} \equiv 0
\end{aligned}
$$

Caso $M$ seja uma variedade Riemanniana, usando a equação (2.4) e a relação $I_{2} \circ d\left(\varphi_{0}\right)_{\gamma(t)}=$ $d\left(I_{2} \circ \varphi_{0}\right)_{\gamma(t)}=d \varphi_{\gamma(t)} \circ d i_{\gamma(t)}$, obtemos que

$\left[\left(\frac{d}{d t}\left(\partial_{\dot{Q}} \mathcal{L}\left(\tilde{\varphi}\left(\gamma(t),(i \circ \gamma)^{\prime}(t)\right)\right)\right)-\partial_{Q} \mathcal{L}\left(\tilde{\varphi}\left(\gamma(t),(i \circ \gamma)^{\prime}(t)\right)\right)+\partial_{\dot{Q}} \mathcal{D}\left(\tilde{\varphi}\left(\gamma(t),(i \circ \gamma)^{\prime}(t)\right)\right)\right) \circ d \varphi_{\gamma(t)}\right] \circ d i_{\gamma(t)}=0$,

ou seja, este funcional, que pertence a $T_{\gamma(t)} M^{*}$, tem restrição nula ao subespaço $T_{\gamma(t)} M_{0}$.

Através da métrica Riemanniana, para cada $t$, existe um único vetor em $T_{\gamma(t)} M$ que representa este funcional. Este é dado simplesmente, sem fazer menção à carta, por

$$
\frac{d}{d t}\left(\frac{\partial \mathcal{L}}{\partial \dot{Q}}\left(\gamma(t), \gamma^{\prime}(t)\right)\right)-\frac{\partial \mathcal{L}}{\partial Q}\left(\gamma(t), \gamma^{\prime}(t)\right)+\frac{\partial \mathcal{D}}{\partial \dot{Q}}\left(\gamma(t), \gamma^{\prime}(t)\right) \in T_{\gamma(t)} M_{0}^{\perp}
$$

Portanto, a equação (2.4) é equivalente a:

$$
\pi_{\gamma(t)}\left(\frac{d}{d t}\left(\frac{\partial \mathcal{L}}{\partial \dot{Q}}\left(\gamma(t), \gamma^{\prime}(t)\right)\right)-\frac{\partial \mathcal{L}}{\partial Q}\left(\gamma(t), \gamma^{\prime}(t)\right)+\frac{\partial \mathcal{D}}{\partial \dot{Q}}\left(\gamma(t), \gamma^{\prime}(t)\right)\right)=0 \quad \forall t
$$

onde $\pi_{q}: T_{q} M \longrightarrow T_{q} M_{0}$ é a projeção ortogonal, $\forall q \in M_{0}$.

Caso $M=\mathbb{R}^{n}$ com a métrica Euclidiana, a carta que consideramos é $\varphi=i d: \mathbb{R}^{n} \longrightarrow \mathbb{R}^{n}$. Daqui em diante, se não for dito o contrário, estaremos restringidos a este caso. 
Dadas as funções $\mathcal{L}$ e $\mathcal{D}$, considere a função energia $E: U \times \mathbb{R}^{n} \longrightarrow \mathbb{R}$,

$$
E(Q, \dot{Q}):=\left\langle\frac{\partial \mathcal{L}}{\partial \dot{Q}}(Q, \dot{Q}), \dot{Q}\right\rangle-\mathcal{L}(Q, \dot{Q}) .
$$

Ao longo de uma solução do sistema, $\gamma: I \longrightarrow M_{0}$,

$$
\begin{gathered}
\frac{d}{d t} E\left(\gamma(t), \gamma^{\prime}(t)\right)=\left\langle\frac{d}{d t}\left(\frac{\partial \mathcal{L}}{\partial \dot{Q}}\left(\gamma(t), \gamma^{\prime}(t)\right)\right), \gamma^{\prime}(t)\right\rangle+\left\langle\frac{\partial \mathcal{L}}{\partial \dot{Q}}\left(\gamma(t), \gamma^{\prime}(t)\right), \gamma^{\prime \prime}(t)\right\rangle \\
-\left\langle\frac{\partial \mathcal{L}}{\partial Q}\left(\gamma(t), \gamma^{\prime}(t)\right), \gamma^{\prime}(t)\right\rangle-\left\langle\frac{\partial \mathcal{L}}{\partial \dot{Q}}\left(\gamma(t), \gamma^{\prime}(t)\right), \gamma^{\prime \prime}(t)\right\rangle=-\left\langle\frac{\partial \mathcal{D}}{\partial \dot{Q}}\left(\gamma(t), \gamma^{\prime}(t)\right), \gamma^{\prime}(t)\right\rangle,
\end{gathered}
$$

sendo que na última igualdade, usamos (2.5) e que para todo $t, \gamma^{\prime}(t) \in T_{\gamma(t)} M_{0}$.

Frequentemente nos modelos, ocorre que a função dissipação é homogênea de grau 2 na segunda variável. Ou seja, $\forall \lambda, \mathcal{D}(Q, \lambda \dot{Q})=\lambda^{2} \mathcal{D}(Q, \dot{Q})$. Neste caso, decorre (Teorema de Euler),

$$
\frac{d}{d t} E\left(\gamma(t), \gamma^{\prime}(t)\right)=-2 \mathcal{D}\left(\gamma(t), \gamma^{\prime}(t)\right)
$$

Este cálculo justifica a associação que se faz da função $\mathcal{D}$ com a potência dissipada pelo sistema.

Observe ainda que se a Lagrangiana tem sua forma mais comum: $\mathcal{L}(Q, \dot{Q})=T(Q, \lambda \dot{Q})-V(Q)$, com a energia cinética também quadrática nas velocidades: $T(Q, \lambda \dot{Q})=\lambda^{2} T(Q, \dot{Q})$, decorre que a energia fica na forma

$$
E(Q, \dot{Q})=\left.\frac{d}{d \lambda}\right|_{\lambda=1} T(Q, \lambda \dot{Q})-T(Q, \dot{Q})+V(Q)=T(Q, \dot{Q})+V(Q) .
$$

Note que estas condições de homogeneidade são independentes das coordenadas adotadas.

\subsubsection{Teorema de Noether}

É necessário adaptar as ideias envolvidas no Teorema de Noether da Mecânica Clássica para os sistemas com dissipação que estamos lidando. Para isto, apresentamos a versão elementar deste resultado o qual será utilizado algumas vezes no próximo capítulo.

Seja $M$ uma variedade, $G$ um grupo de Lie e $\Phi: G \times M \longrightarrow M$ uma ação suave. Diremos que um sistema dissipativo sobre $M$, com Lagrangiana $\mathcal{L}$ e função dissipação $\mathcal{D}$, admite uma simetria a um parâmetro $\gamma: I \longrightarrow G$ se:

$$
\mathcal{L}\left(d \Phi_{\gamma(s)}(x, \dot{x})\right)=\mathcal{L}(x, \dot{x}), \quad \partial_{\dot{x}} \mathcal{D}(x, \dot{x})\left(\left.\frac{\partial}{\partial s}\right|_{s=0} \Phi_{\gamma(s)}(x)\right)=0, \quad \forall s \in I,(x, \dot{x}) \in T M,
$$

onde usamos a notação $\Phi_{g}(x):=\Phi(g, x)$, e $d \Phi_{g}(x, \dot{x})=\left(\Phi_{g}(x), D \Phi_{g}(x)[\dot{x}]\right)$.

Teorema 2.3. Se um sistema dissipativo $(M, \mathcal{L}, \mathcal{D})$, com $M \subset \mathbb{R}^{n}$, admite uma simetria $\gamma(s)$ $(\gamma(0)=e$, elemento neutro do grupo), então ele possui a seguinte integral primeira

$$
h(x, \dot{x}):=\partial_{\dot{x}} \mathcal{L}(x, \dot{x})\left(\left.\frac{\partial}{\partial s}\right|_{s=0} \Phi_{\gamma(s)}(x)\right) .
$$

Demonstração. Note que ao longo de uma solução,

$$
\begin{gathered}
\frac{d}{d t} h(x, \dot{x})=\frac{d}{d t}\left(\partial_{\dot{x}} \mathcal{L}(x, \dot{x})\right)\left(\left.\frac{\partial}{\partial s}\right|_{s=0} \Phi_{\gamma(s)}(x)\right)+\partial_{\dot{x}} \mathcal{L}(x, \dot{x})\left(\left.\frac{\partial}{\partial s}\right|_{s=0} D \Phi_{\gamma(s)}(x)[\dot{x}]\right)= \\
=\partial_{x} \mathcal{L}(x, \dot{x})\left(\left.\frac{\partial}{\partial s}\right|_{s=0} \Phi_{\gamma(s)}(x)\right)+\partial_{\dot{x}} \mathcal{L}(x, \dot{x})\left(\left.\frac{\partial}{\partial s}\right|_{s=0} D \Phi_{\gamma(s)}(x)[\dot{x}]\right)-
\end{gathered}
$$




$$
-\partial_{\dot{x}} \mathcal{D}(x, \dot{x})\left(\left.\frac{\partial}{\partial s}\right|_{s=0} \Phi_{\gamma(s)}(x)\right)=\left.\frac{d}{d t} \frac{\partial}{\partial s}\right|_{s=0} \mathcal{L}\left(d \Phi_{\gamma(s)}(x, \dot{x})\right)=0
$$

Observação 2.4. Como se pode notar da demonstração, não são necessárias todas as hipóteses listadas. A simetria da Lagrangiana pode se dar através de uma família a um parâmetro, diferenciável, de aplicações $\psi_{s} \in \mathcal{C}^{\infty}(M)$, com $\psi_{0}=i d_{M}$. A condição de simetria (2.9) também pode ser trocada por uma condição infinitesimal. Porém, esta versão é suficiente para nossos propósitos.

\subsubsection{Equações de movimento em $\mathrm{SO}(3)$}

Será necessário calcular as equações de movimento associadas às variáveis de rotação. Uma maneira de obtê-las de forma livre de coordenadas é através da redução de Euler-Poincaré [HSS09]. Porém, é necessário supor que a Lagrangiana $L: T \mathrm{SO}(3) \longrightarrow \mathbb{R}$ é invariante à esquerda, o que não ocorrerá em todos os casos tratados aqui.

Note que $\mathrm{SO}(3) \subset \mathrm{GL}(3)$, o qual é um aberto do espaço vetorial $\mathrm{M}(3)$ quando o identificamos com $\mathbb{R}^{9}$ agrupando as colunas de cada matriz em sequência (transpostas) para formar um vetor de nove entradas. Esta identificação induz o produto interno em $\mathrm{M}(3)$ :

$$
\langle X, Y\rangle_{\mathrm{GL}(3)}:=\operatorname{Tr}\left(X^{T} Y\right) \quad \forall X, Y \in \mathrm{M}(3) .
$$

Lembre que $\mathrm{SO}(3)$ é um grupo de Lie, com a multiplicação usual de matrizes. Assim, cada espaço tangente é facilmente calculado: $T_{Y} \mathrm{SO}(3) \equiv Y T_{I} \mathrm{SO}(3)=Y$ skew(3), onde skew(3) é o conjunto das matrizes antissimétricas de ordem 3.

Assim, suponha que o sistema seja descrito por uma Lagrangiana que possua uma extensão suave $L: T \mathrm{GL}(3) \longrightarrow \mathbb{R}$. Pela equação (2.5), devemos calcular para cada $Y \in \mathrm{SO}(3)$ a projeção ortogonal $P_{Y}: \mathrm{M}(3) \longrightarrow T_{Y} \mathrm{SO}(3)$, segundo a métrica (2.11).

Lema 2.5. Para todo $Y \in \mathrm{SO}(3)$ e todo $H \in \mathrm{M}(3)$,

$$
P_{Y} H=Y\left(Y^{T} H\right)_{a},
$$

onde $B_{a}:=1 / 2\left(B-B^{T}\right)$ é a parte antissimétrica de uma matriz $B$.

Demonstração. Observe que $f_{Y}: \mathrm{M}(3) \longrightarrow \mathrm{M}(3), f_{Y}(H):=Y H$, é uma isometria para todo $Y \in \mathrm{SO}(3)$. Portanto, a partir da decomposição ortogonal $\mathrm{M}(3)=\operatorname{skew}(3) \oplus \operatorname{Sym}(3)$, obtemos a decomposição ortogonal mais geral $\mathrm{M}(3)=Y$ skew $(3) \oplus Y \operatorname{Sym}(3)=T_{Y} \mathrm{SO}(3) \oplus Y \operatorname{Sym}(3)$. Desde que $H=Y\left(\left(Y^{T} H\right)_{a}+\left(Y^{T} H\right)_{s}\right), \forall H \in \mathrm{M}(3)$, a conclusão do lema segue.

Suponhamos que a Lagrangiana seja da forma usual, diferença entre energia cinética e potencial, $\mathcal{L}=T-V$, de forma que $V$ e $\mathcal{D}$ não dependam de $\dot{Y}$. Assuma que $T: T$ GL $(3) \longrightarrow \mathbb{R}$ seja invariante à esquerda, ou seja, que exista $K: \mathrm{M}(3) \longrightarrow \mathbb{R}$, tal que $T(Y, \dot{Y})=K\left(Y^{-1} \dot{Y}\right)$, para todo $(Y, \dot{Y}) \in T \mathrm{GL}(3)$. Desta forma, vale a identidade a seguir.

Lema 2.6. Ao longo de toda curva suave $Y(t) \in \mathrm{SO}(3),(Y, \dot{Y}) \in T \mathrm{SO}(3)$,

$$
P_{Y}\left(\frac{d}{d t}\left(\frac{\partial T}{\partial \dot{Y}}\right)-\frac{\partial T}{\partial Y}\right)=Y\left(\frac{d W}{d t}(t)+[\Omega, W(t)]\right)
$$

onde $\Omega=Y^{T} \dot{Y} \in$ skew $(3)$ e $W(t)=\left(\frac{\partial K}{\partial \Omega}\right)_{a}$. 
Demonstração. Dados, $Y \in \mathrm{SO}(3), \dot{Y} \in T_{Y} \mathrm{SO}(3)$ e um arbitrário $H \in \mathrm{M}(3)$, temos

$$
\begin{aligned}
\left\langle\frac{d}{d t}\left(\frac{\partial T}{\partial \dot{Y}}\right)-\frac{\partial T}{\partial Y}, H\right\rangle & =\frac{d}{d t}\left(\left.\frac{d}{d \lambda}\right|_{\lambda=0} K\left(Y^{T}(\dot{Y}+\lambda H)\right)\right) \\
& -\left.\frac{d}{d \lambda}\right|_{\lambda=0} K\left((\dot{Y}+\lambda H)^{T} \dot{Y}\right)=\left\langle Y \frac{d}{d t}\left(\frac{\partial K}{\partial \Omega}\right)+\dot{Y}\left(\frac{\partial K}{\partial \Omega}-\frac{\partial K^{T}}{\partial \Omega}\right), H\right\rangle,
\end{aligned}
$$

$\log \mathrm{e}$

$$
\frac{d}{d t}\left(\frac{\partial T}{\partial \dot{Y}}\right)-\frac{\partial T}{\partial Y}=Y\left(\frac{d}{d t}\left(\frac{\partial K}{\partial \Omega}\right)+2 \Omega W(t)\right)
$$

e o resultado segue do Lema 2.5.

Desta forma, de (2.5), as equações para a rotação ficam:

$$
P_{Y}\left(\frac{d}{d t}\left(\frac{\partial T}{\partial \dot{Y}}\right)-\frac{\partial T}{\partial Y}\right)+P_{Y}\left(\frac{\partial V}{\partial Y}\right)=0
$$

Portanto,

$$
\frac{d W}{d t}(t)+[\Omega, W(t)]=-\left(Y^{T} \frac{\partial V}{\partial Y}\right)_{a}
$$

\subsubsection{Equações de movimento para o corpo girante}

Passemos ao problema de um corpo contínuo em rotação livre. Como descrito na Seção 2.1, a variedade que representa seu movimento é $\mathrm{SO}(3) \times \mathbb{R}^{3} \times \mathcal{C} \subset \mathrm{GL}(3) \times \mathbb{R}^{3} \times \mathcal{C}$. Neste caso, tomemos a métrica produto

$$
\left\langle\left(X, x, h_{1}\right),\left(Y, y, h_{2}\right)\right\rangle_{\mathrm{GL}(3) \times \mathbb{R}^{3} \times \mathcal{C}}:=\operatorname{Tr}\left(X^{T} Y\right)+x \cdot y+\int_{\mathcal{B}} h_{1}(x) h_{2}(x) d x .
$$

Desta forma, a projeção ortogonal fica $\pi_{(Y, y, u)}=\left(P_{Y} \times \operatorname{Id}_{\mathbb{R}^{3}} \times \operatorname{Id}_{\mathcal{V}}\right): \mathrm{M}(3) \times \mathbb{R}^{3} \times \mathcal{V} \longrightarrow$ $T_{Y} \mathrm{SO}(3) \times \mathbb{R}^{3} \times \mathcal{V}$. Façamos a hipótese de que a deformação do corpo seja pequena em relação às suas dimensões. Ou seja, definindo $\phi: \mathbb{R} \times \mathcal{B} \longrightarrow \mathbb{R}^{3}$, onde $\phi(t, x)$ é a posição do ponto $x$ do corpo (não deformado), no referencial inercial, no instante $t$, assumimos que $\phi(t, x)=Y(x+u(t, x))+y(t)$ onde $\|u\| \approx 0$. Assim, por (2.1), temos:

$$
\partial_{t} \phi(t, x)=Y\left(\Omega(x+u)+u_{t}\right)+\dot{y} .
$$

A equação de Euler-Lagrange (com dissipação) para esta variedade se decompõe nas três seguintes:

$$
\begin{gathered}
\frac{d W}{d t}(t)+[\Omega, W(t)]=-\left(Y^{T} \frac{\partial V}{\partial Y}\right)_{a}, \\
\frac{d}{d t}\left(\frac{\delta \mathcal{L}}{\delta v}\right)-\frac{\delta \mathcal{L}}{\delta u}+\frac{\delta \mathcal{D}}{\delta v}=0 \\
\frac{d}{d t}\left(\frac{\partial \mathcal{L}}{\partial \dot{y}}\right)-\frac{\partial \mathcal{L}}{\partial y}=0
\end{gathered}
$$

onde $v=u_{t}:=\partial_{t} u$ e fazendo a hipótese de que a função dissipação $\mathcal{D}$ não depende de $\dot{y}$ e $\dot{Y}$. Todas as derivadas nesta fórmula indicam gradientes em relação às métricas de (2.15). O símbolo $\delta$ da segunda equação indica que a derivada é tomada no espaço de funções.

Observação 2.7. Como já dissemos, em [BL87] e [BL91] não foi definido com detalhe o espaço de funções $\mathcal{C}$. O trataremos de forma parecida ao cálculo de variações. 
A Lagrangiana do sistema tem a forma: $\mathcal{L}: T \mathrm{SO}(3) \times \mathbb{R}^{6} \times(\mathcal{C} \times \mathcal{V}) \longrightarrow \mathbb{R}$, com

$$
\mathcal{L}\left(Y, y, u, \dot{Y}, \dot{y}, u_{t}\right)=T\left(Y, y, u, \dot{Y}, \dot{y}, u_{t}\right)-V(Y, y, u) .
$$

Portanto, a energia cinética é obtida de (2.16)

$$
T=\frac{1}{2} \int_{\mathcal{B}}\left\|\partial_{t} \phi(t, x)\right\|^{2} \rho(x) d x=\frac{1}{2} \int_{\mathcal{B}}\left\|\Omega(t)(x+u(t, x))+u_{t}(t, x)+Y(t)^{T} \dot{y}(t)\right\|^{2} \rho(x) d x .
$$

A energia potencial $V$ depende das características internas do corpo em consideração, sendo suposto em geral que $V$ é constante relativamente às variáveis $\dot{Y}, \dot{y}, u_{t}$.

Procedendo com os cálculos, obtemos o seguinte teorema apresentado em [BL87].

Teorema 2.8. (Baillieul \&3 Levi, 1987)

A evolução temporal do sistema mecânico em consideração, com energia potencial $V$ e função dissipação $\mathcal{D}$ é solução do seguinte sistema de equações

$$
\begin{gathered}
\frac{d W}{d t}(t)+[\Omega, W(t)]+\int_{\mathcal{B}} \rho\left(Y^{T} \ddot{y} u^{T}\right)_{a} d x=-\left(Y^{T} \frac{\partial V}{\partial Y}\right)_{a}, \\
\rho(x)\left(u_{t t}+2 \Omega u_{t}+\dot{\Omega}(x+u)+\Omega^{2}(x+u)+Y^{T} \ddot{y}\right)=-\frac{\delta V}{\delta u}-\frac{\delta \mathcal{D}}{\delta v}, \\
\int_{\mathcal{B}}\left(u_{t t}+\Omega^{2} u+2 \Omega u_{t}+\dot{\Omega} u+Y^{T} \ddot{y}\right) \rho d x=-Y^{T} \frac{\partial V}{\partial y}
\end{gathered}
$$

onde $W(t)$ foi definido no Lema 2.6.

Considere o isomorfismo: $S: \operatorname{skew}(3) \longrightarrow \mathbb{R}^{3}$,

$$
S(B)=S\left(\left(\begin{array}{ccc}
0 & -b_{3} & b_{2} \\
b_{3} & 0 & -b_{1} \\
-b_{2} & b_{1} & 0
\end{array}\right)\right)=\left(\begin{array}{l}
b_{1} \\
b_{2} \\
b_{3}
\end{array}\right)
$$

O seguinte lema é facilmente verificado escrevendo-se explicitamente em componentes.

Lema 2.9. Dados $A, B \in \operatorname{skew}(3)$ e $w_{1}, w_{2} \in \mathbb{R}^{3}$, valem as seguintes identidades:

(i) $S([A, B])=S(A) \times S(B)$.

(ii) $S\left(w_{1} w_{2}^{T}-w_{2} w_{1}^{T}\right)=w_{2} \times w_{1}$, e portanto, $S\left(\left(w_{1} w_{2}^{T}\right)_{a}\right)=\frac{1}{2} w_{2} \times w_{1}$.

(iii) $A w_{1}=S(A) \times w_{1}$.

Assim, através deste isomorfismo, a equação (2.20) fica na forma

$$
\rho(x)\left(u_{t t}+2 \omega \times u_{t}+\dot{\omega} \times(x+u)+\omega \times(\omega \times(x+u))+Y^{T} \ddot{y}\right)=-\frac{\delta V}{\delta u}-\frac{\delta \mathcal{D}}{\delta v},
$$

sendo $\omega(t):=S(\Omega)$, a velocidade angular no referencial do corpo.

Observação 2.10. Tome $u(t, x) \equiv 0, \forall t, x$ e $V, \mathcal{D} \equiv 0$ (já que não há deformação) e $y \equiv 0$. Dessa forma, (2.20) e (2.21) não fazem parte das equações de movimento, e (2.19) coincide com a equação de movimento do corpo rígido livre. 


\subsubsection{O caso incompressível}

É hipótese usual em mecânica do contínuo que o corpo em movimento se deforme, mas não varie o volume de suas partes. Em termos precisos, seja a função $\phi(t, x)$ aquela que fornece a posição do ponto que em $t=0$ estava em $x \in \mathcal{B}$. Impomos que para qualquer aberto $U \subset \mathcal{B}, \operatorname{vol}(\phi(t, U))=$ $\operatorname{vol}(U)$. Neste caso, dizemos que o corpo é incompressivel. Da seção anterior, estabelecemos que $\phi(t, x)=Y(t)(x+u(t, x))+y(t)$. Portanto, o corpo é incompressível se, e só se, $\psi(x):=x+u(t, x)$ também preserva volume. Lembrando que consideramos apenas pequenas deformações, para todo $\varepsilon \approx 0 \mathrm{impomos}$ que $\operatorname{det}\left(D_{x} \phi(t, x)\right)=\operatorname{det}\left(\operatorname{Id}+\varepsilon D_{x} u(t, x)\right)=1$, portanto,

$$
0=\left.\frac{\partial}{\partial \varepsilon}\right|_{\varepsilon=0} \operatorname{det}\left(\operatorname{Id}+\varepsilon D_{x} u(t, x)\right)=\operatorname{div} u(t, x) .
$$

Este vínculo, div $u=0$, restringe o espaço de funções discutido anteriormente para $\mathcal{C}_{0}\left(\mathcal{B}, \mathbb{R}^{3}\right) \subset$ $\mathcal{C}\left(\mathcal{B}, \mathbb{R}^{3}\right)$, o qual claramente também é vetorial, de forma que também podemos aplicar facilmente o Teorema 2.2 a ele.

Para isso, basta calcularmos qual é a projeção ortogonal (segundo o produto interno $L^{2}(\mathcal{B})$, ver (2.15)) $P: \mathcal{C}\left(\mathcal{B}, \mathbb{R}^{3}\right) \longrightarrow \mathcal{C}_{0}\left(\mathcal{B}, \mathbb{R}^{3}\right)$. Os demais cálculos seguem como na seção anterior.

Lembramos assim do Teorema da Decomposição de Helmholtz [AMR07].

Teorema 2.11. Qualquer campo vetorial suave $X$ sobre uma variedade Riemanniana compacta $(M, g)$ se decompõe, unicamente, como

$$
X=Y-\nabla p,
$$

sendo que $Y$ tem divergência nula e é paralelo a $\partial M$ e p $: M \longrightarrow \mathbb{R}$ é uma função suave.

Afirmamos que a aplicação $X \mapsto Y=X+\nabla p$ é a projeção que estamos procurando. De fato, é simples verificar que é linear. Só resta mostrar que a decomposição acima é ortogonal. Tomando $M=\overline{\mathcal{B}} \subset \mathbb{R}^{3}$ com a métrica Euclidiana, $X, Y$ campos em $\overline{\mathcal{B}}$ e $p \in C^{\infty}(\mathcal{B})$, como no teorema, temos

$$
\int_{\mathcal{B}}\langle Y(x), \nabla p(x)\rangle d x=\int_{\mathcal{B}} \operatorname{div}(p(x) Y(x)) d x=\int_{\partial \mathcal{B}} p(x) Y(x) \cdot \hat{n}(x) d S=0 .
$$

No cálculo usamos o Teorema de Gauss e as conclusões do Teorema 2.11. Desta forma garantimos que a projeção é dada por:

$$
P(X)=X+\nabla p
$$

notando que $p(x)$ é obtido através de $X(x)$ pela equação de Poisson $\Delta p=\operatorname{div} X$.

Em resumo, em caso de incompressibilidade devemos somente aplicar a projeção (2.24) na equação (2.23). Neste caso, esta equação fica:

$$
\rho(x)\left(u_{t t}+2 \omega \times u_{t}+\dot{\omega} \times(x+u)+\omega \times(\omega \times(x+u))+Y^{T} \ddot{y}\right)=-\frac{\delta V}{\delta u}-\frac{\delta \mathcal{D}}{\delta v}-\nabla p .
$$

\subsubsection{Potenciais Elástico e Gravitacional}

Passemos ao estudo dos dois termos restantes da equação (2.25). Suponhamos que o corpo seja viscoelástico, ou seja, que apresente resistência à deformação e que perca energia ao realizar movimentos internos.

Em geral, objetos sólidos possuem densidade de massa não homogênea, o que usualmente indica diferente resistência à deformação ao longo de sua extensão. Além de possuir articulações, que se movimentam e dissipam energia de forma completamente distinta de outras partes.

Nesta seção, os objetos de estudo são corpos homogêneos que possuem elasticidade tridimensional linear, ou seja, que se deformam (dissipando energia) em todas as direções do espaço, não possuindo parte rígida, de forma que suas partes se comportem como um sistema massa-mola, cujas 
forças são lineares no deslocamento e na velocidade (respectivamente). Tomemos ainda o ponto de referência do corpo no centro de massa, eliminando a coordenada de movimento, $y \equiv 0$.

Este conceito análogo a força, sobre cada elemento do corpo, é desempenhado através dos chamados tensores de tensão e de tensão viscosa. Exigindo sua linearidade, obtemos as funções potencial e de dissipação (consultar [LL86]):

$$
\begin{aligned}
V_{e l}(Y, u, y) & :=\int_{\mathcal{B}}\left[\frac{\mu}{2}\left(\left(\partial_{i} u_{k}\right)^{2}+\partial_{k} u_{i} \partial_{i} u_{k}\right)+\left(\frac{K}{2}-\frac{\mu}{3}\right)\left(\partial_{l} u_{l}\right)^{2}\right] d V, \\
\mathcal{D}(\dot{Y}, v, \dot{y}) & :=\int_{\mathcal{B}}\left[\frac{\eta}{2}\left(\left(\partial_{i} v_{k}\right)^{2}+\partial_{k} v_{i} \partial_{i} v_{k}\right)+\left(\frac{\zeta}{2}-\frac{\eta}{3}\right)\left(\partial_{l} v_{l}\right)^{2}\right] d V,
\end{aligned}
$$

onde $K, \mu$ são os módulos volumétrico e de cisalhamento elásticos, respectivamente, e $\zeta, \eta$ são os módulos volumétrico e de cisalhamento viscosos, respectivamente. Observamos que foi feito uso da notação de soma.

Defina, os tensores de tensão e de tensão viscosa, respectivamente, através de suas componentes:

$$
\begin{gathered}
\sigma_{i k}:=\left(\left(K-\frac{2 \mu}{3}\right) \operatorname{div} u\right) \delta_{i k}+\mu\left(\partial_{i} u_{k}+\partial_{k} u_{i}\right), \\
\tilde{\sigma}_{i k}:=\left(\left(\zeta-\frac{2 \eta}{3}\right) \operatorname{div} v\right) \delta_{i k}+\eta\left(\partial_{i} v_{k}+\partial_{k} v_{i}\right) .
\end{gathered}
$$

Para o significado físico destes objetos, consultar a referência [LL86] (parágrafo 4 para o primeiro, e parágrafo 34 para o segundo).

Exigiremos que tais tensores tenham componente normal nula sobre o bordo. Esta é uma condição típica, que será exigida valer para todas as deformações do espaço $\mathcal{C}$. Logo,

$$
\begin{gathered}
(\sigma n(x))_{i}=\left(\left(K-\frac{2 \mu}{3}\right) \operatorname{div} u(t, x)\right) n_{i}(x)+\mu\left(\partial_{i} u_{k}(t, x)+\partial_{k} u_{i}(t, x)\right) n_{k}(x)=0 \\
(\tilde{\sigma} n(x))_{i}=\left(\left(\zeta-\frac{2 \eta}{3}\right) \operatorname{div} v(t, x)\right) n_{i}(x)+\eta\left(\partial_{i} v_{k}(t, x)+\partial_{k} v_{i}(t, x)\right) n_{k}(x)=0
\end{gathered}
$$

para todo $x \in \partial \mathcal{B}$, e para todo $t$, onde $n(x):=\left(n_{1}(x), n_{2}(x), n_{3}(x)\right)$ é o vetor normal (exterior) a $\partial \mathcal{B}$.

Desta forma, dados $u \in \mathcal{C}$ e $h \in \mathcal{V}$, defina $\varphi_{\varepsilon}=u+\varepsilon h$. Portanto,

$$
\begin{aligned}
\delta_{u} V_{e l}(h)=\left.\frac{\partial}{\partial \varepsilon}\right|_{\varepsilon=0} \int_{\mathcal{B}}\left[\frac{\mu}{2}\left(\left(\partial_{i}\left(\varphi_{\varepsilon}\right)_{k}\right)^{2}+\left(\partial_{k}\left(\varphi_{\varepsilon}\right)_{i}\right)\left(\partial_{i}\left(\varphi_{\varepsilon}\right)_{k}\right)\right)+\left(\frac{K}{2}-\frac{\mu}{3}\right)\left(\partial_{l}\left(\varphi_{\varepsilon}\right)_{l}\right)^{2}\right] d V \\
=\int_{\mathcal{B}}\left[\mu\left(\partial_{i} u_{k} \partial_{i} h_{k}+\partial_{i} u_{k} \partial_{k} h_{i}\right)+\left(K-\frac{2 \mu}{3}\right) \partial_{l} u_{l} \partial_{j} h_{j}\right] d V \\
=\int_{\mathcal{B}}\left[\mu\left(\left\langle\nabla u_{i}, \nabla h_{i}\right\rangle+\left\langle\nabla h_{i}, \partial_{i} u\right\rangle\right)+\left(K-\frac{2 \mu}{3}\right)(\operatorname{div} u)(\operatorname{div} h)\right] d V .
\end{aligned}
$$

Mas, por uma identidade vetorial elementar e pelo Teorema de Gauss, para todos $\alpha$ campo escalar e $w$ campo vetorial,

$$
\int_{\mathcal{B}}\langle\nabla \alpha, w\rangle+\alpha \operatorname{div} w d V=\int_{\mathcal{B}} \operatorname{div}(\alpha w) d V=\int_{\partial \mathcal{B}} \alpha\langle w, n\rangle d S .
$$

Logo, fazendo $\alpha=h_{i}$ e $w=\sigma_{i}$, a $i$-ésima coluna do tensor $\sigma$, decorre que

$$
\begin{gathered}
\left\langle\sigma_{i}, \nabla h_{i}\right\rangle=\sigma_{i k} \partial_{k} h_{i}=\left(\left(K-\frac{2 \mu}{3}\right) \operatorname{div} u\right) \partial_{i} h_{i}+\mu\left(\left\langle\partial_{i} u, \nabla h_{i}\right\rangle+\left\langle\nabla u_{i}, \nabla h_{i}\right\rangle\right) \\
=\left(K-\frac{2 \mu}{3}\right)(\operatorname{div} u)(\operatorname{div} h)+\mu\left(\left\langle\partial_{i} u, \nabla h_{i}\right\rangle+\left\langle\nabla u_{i}, \nabla h_{i}\right\rangle\right) .
\end{gathered}
$$


Mas, da identidade citada:

$$
\int_{\mathcal{B}}\left\langle\nabla h_{i}, \sigma_{i}\right\rangle+h_{i} \nabla \sigma_{i} d V=\int_{\partial \mathcal{B}} h_{i}\left\langle\sigma_{i}, n\right\rangle d S=0
$$

pela condição de contorno (2.29).

Mas, constata-se que $\operatorname{div} \sigma_{i}=\partial_{k} \sigma_{i k}=\left(K+\frac{\mu}{3}\right) \partial_{i}(\operatorname{div} u)+\mu \Delta u_{i}$. Logo,

$$
h_{i} \operatorname{div} \sigma_{i}=\left(K+\frac{\mu}{3}\right)\langle\nabla(\operatorname{div} u), h\rangle+\mu\langle\Delta u, h\rangle .
$$

Portanto,

$$
\delta_{u} V_{e l}(h)=\int_{\mathcal{B}}\left\langle\sigma_{i}, \nabla h_{i}\right\rangle d V=-\int_{\mathcal{B}}\left\langle\left(K+\frac{\mu}{3}\right) \nabla(\operatorname{div} u)+\mu \Delta u, h\right\rangle d V, \quad \forall h \in \mathcal{V} .
$$

Desta forma, concluímos que:

$$
\frac{\delta V_{e l}}{\delta u}=-\left(K+\frac{\mu}{3}\right) \nabla(\operatorname{div} u)-\mu \Delta u,
$$

a força elástica para um sólido (compressível) segundo o modelo de Kelvin-Voigt. Compare, por exemplo, com a equação (19) do capítulo $\mathrm{V}$ de [Lov44] (em relação aos coeficientes de Lamé, $K=\lambda+2 \mu / 3)$.

Analogamente, para a força viscosa encontramos:

$$
\frac{\delta \mathcal{D}}{\delta v}=-\left(\zeta+\frac{\eta}{3}\right) \nabla(\operatorname{div} v)-\zeta \Delta v
$$

Note que (consultar [LL87], parágrafo 15) este termo que expressa a viscosidade do meio é análogo ao que ocorre na equação de Navier-Stokes para fluidos isotrópicos, compressíveis. Caso o material seja incompressível, os primeiros termos de cada expressão se anulam, fazendo com que os módulos volumétricos não constem nas equações. De fato, desconsiderando as interações elásticas pode-se estender as ideias apresentadas até aqui para um fluido incompressível, sendo o conjunto de funções trocado pelo conjunto de difeormorfismos $\mathcal{C}=\left\{\phi: \mathcal{B} \longrightarrow \mathbb{R}^{3}, \operatorname{det}(D \phi(x))=1\right\}$. O artigo [Arn66] é o trabalho pioneiro sobre esta abordagem. Neste contexto, a função dissipação para o fluido pode ser encontrada em [Bat00], equação (3.4.5). Notamos que ela coincide com (2.27) no caso de pequenas deformações e $\operatorname{div} u=0$.

Consideremos agora a influência da atração gravitacional entre as partes de $\mathcal{B}$. Ela é expressa através do potencial,

$$
V_{s g}(u)=-\frac{G}{2} \int_{\mathcal{B}} \int_{\mathcal{B}} \frac{\rho(x) \rho(y)}{\|x+u(x)-(y+u(y))\|} d x d y .
$$

De forma análoga calculamos sua derivada:

$$
\begin{gathered}
\delta_{u} V_{s g}(h)=\frac{G}{2} \int_{\mathcal{B}} \int_{\mathcal{B}} \rho(x) \rho(y) \frac{\langle x-y+u(x)-u(y), h(x)-h(y)\rangle}{\|x-y+u(x)-u(y)\|^{\frac{3}{2}}} d x d y \\
=G \int_{\mathcal{B}}\left\langle\rho(x) \int_{\mathcal{B}} \rho(y) \frac{x-y+u(x)-u(y)}{\|x-y+u(x)-u(y)\|^{\frac{3}{2}}} d y, h(x)\right\rangle d x,
\end{gathered}
$$

onde usamos mudanças de variáveis e o Teorema de Fubini.

Logo,

$$
\frac{\delta V_{s g}}{\delta u}(x)=-G \rho(x) \int_{\mathcal{B}} \frac{x-y+u(x)-u(y)}{\|x-y+u(x)-u(y)\|^{\frac{3}{2}}} \rho(y) d y .
$$


Desta forma, a equação (2.25) fica

$u_{t t}+2 \omega \times u_{t}+\dot{\omega} \times(x+u)+\omega \times(\omega \times(x+u))=\frac{\mu}{\rho} \Delta u+G \int_{\mathcal{B}} \frac{x-y+u(x)-u(y)}{\|x-y+u(x)-u(y)\|^{\frac{3}{2}}} \rho(y) d y+\frac{\zeta}{\rho} \Delta v-\frac{\nabla p}{\rho}$.

Considere agora somente suas soluções de equilíbrio relativo, $\dot{\omega} \equiv 0$ e $v \equiv 0$. Suponha que a rotação se dê em torno do eixo $z$. Assim, obtemos a equação

$$
-\omega^{2}\left(x_{1}+u_{1}, x_{2}+u_{2}, 0\right)=\frac{\mu}{\rho} \Delta u+G \int_{\mathcal{B}} \frac{x-y+u(x)-u(y)}{\|x-y+u(x)-u(y)\|^{\frac{3}{2}}} \rho(y) d y-\frac{1}{\rho} \nabla p .
$$

Esta é aproximadamente a equação usada em [Lov44], artigo 177, para obter os números de Love associados a uma esfera elástica gravitante incompressível. No livro, é considerada a equação:

$$
-\omega^{2} \rho(x)\left(x_{1}, x_{2}, 0\right)=\mu \Delta u(x)+\rho(x) \nabla V(x)-\nabla p, \quad \forall x \in \mathcal{B},
$$

sendo,

$$
V(x)=-G \int_{\phi(\mathcal{B})} \frac{\rho(y)}{\|x-y\|} d y .
$$

Como vimos nas Seções 1.1.1 e 1.1.2, em geral estes potenciais não são tratados exatamente. Eles são expandidos em harmônicos esféricos, e estuda-se a contribuição de cada modo normal na resposta do corpo.

\subsection{Teoremas gerais}

Nesta seção, expomos alguns resultados clássicos da Teoria das Equações Diferenciais Ordinárias que serão necessários na análise dos modelos do Capítulo 3.

\subsubsection{Princípio de LaSalle}

Devido à existência de uma função de Lyapounov em todos os modelos tratados no texto, enunciamos o Teorema de LaSalle, cuja demonstração pode ser encontrada em [Hal80].

Dada uma equação diferencial autônoma,

$$
\dot{x}=f(x)
$$

sendo $f: U \subset \mathbb{R}^{n} \longrightarrow \mathbb{R}^{n}$ campo suave sobre o aberto $U$, uma função $E: \mathbb{R}^{n} \longrightarrow \mathbb{R}$ será chamada de função de Lyapounov para (2.33) sobre $U$ se $E$ for diferenciável em $U$, contínua em $\bar{U}$ e

$$
\dot{E}(x):=\langle\nabla E(x), f(x)\rangle \leq 0, \quad \forall x \in U .
$$

Seja

$$
S:=\{x \in U: \dot{E}(x)=0\},
$$

e $M \subset S$ o maior subconjunto invariante pelo fluxo de (2.33). Podemos formular assim o princípio de LaSalle:

Teorema 2.12. Se E é função de Lyapounov em $U, x_{0} \in U$, e sua respectiva órbita (positiva) $\gamma^{+}\left(x_{0}\right)$ está completamente contida em $K \subset U$ compacto, então seu $\omega$-limite está contido em $M$.

\subsubsection{Perturbação Singular}

Serão necessários alguns resultados de Teoria da Perturbação Singular na análise de sistemas presentes no texto. Seguiremos as definições e resultados da seção 2.1 de [BG06]. 
Entendemos por um sistema lento-rápido um sistema de equações diferenciais ordinárias em duas variáveis $x \in \mathbb{R}^{n}, y \in \mathbb{R}^{m}$ e um parâmetro $\varepsilon>0$, considerado pequeno. Sendo $f: U \subset$ $\mathbb{R}^{n+m} \times \mathbb{R} \longrightarrow \mathbb{R}^{n}$, e $g: U \subset \mathbb{R}^{n+m} \times \mathbb{R} \longrightarrow \mathbb{R}^{m}$ funções suaves, tal sistema deve ter a forma

$$
\begin{gathered}
\dot{x}=f(x, y, \varepsilon), \\
\dot{y}=\varepsilon g(x, y, \varepsilon) .
\end{gathered}
$$

Tomando uma reescala de tempo, $\tau=\varepsilon t$, e denotando por $x^{\prime}, y^{\prime}$ as derivadas das funções relativas a este novo parâmetro, o sistema (2.34) se torna

$$
\begin{gathered}
\varepsilon x^{\prime}=f(x, y, \varepsilon), \\
y^{\prime}=g(x, y, \varepsilon) .
\end{gathered}
$$

O sistema (2.34) é denominado sistema rápido e (2.35) o sistema lento associado, pois desde que assumimos $\varepsilon \approx 0, \tau$ varia lentamente com grandes variações de $t$.

Pensando nestes sistemas como perturbações do caso $\varepsilon=0$, considere como aproximação de (2.34) o sistema obtido anulando-se somente o $\varepsilon$ que multiplica a função $g$. Neste caso $y(t)=y_{0}$, $\forall t$, portanto a variável $x$ deve ser solução da equação autônoma

$$
\dot{x}=f\left(x, y_{0}, \varepsilon\right) .
$$

Neste caso, $y_{0}$ se comporta como um parâmetro, como se sua variação fosse tão lenta que $x(t)$ não a sente. Por esta razão $x$ é chamada de variável rápida e y variável lenta. A solução de (2.36) é denominada a média rápida de (2.34), a qual nos dará informações sobre o sistema completo.

Aplicando a mesma ideia a (2.35) obtemos o vínculo

$$
f(x, y, \varepsilon)=0 .
$$

Se for possível, mesmo que localmente, obter (2.37) como o gráfico de uma função $x=x^{\star}(y)$, chamaremos de média lenta de (2.35) a solução de

$$
y^{\prime}=g\left(x^{\star}(y), y, \varepsilon\right),
$$

e $x(\tau)=x^{\star}(y(\tau))$. Note que $x^{\star}$ também depende implicitamente de $\varepsilon$.

Assim, a média lenta $(x(\tau), y(\tau))$ deixa invariante o conjunto $\mathcal{M}:=\left\{(x, y): x=x^{\star}(y), y \in V\right\}$, o qual denominaremos variedade lenta. Diremos que esta variedade é uniformemente assintoticamente estável se as partes reais de todos os autovalores de $A^{\star}(y)$ são negativas, e não estão arbitrariamente próximas de zero, para todo $y \in V$, onde

$$
A^{\star}(y):=\frac{\partial f}{\partial x}\left(x^{\star}(y), y, \varepsilon\right) .
$$

Lembre que estamos anulando somente uma das ocorrências de $\varepsilon$ em (2.34), (2.35), sendo que nas demais $\varepsilon$ é considerada uma variável muda. O Teorema 2.13 garante a persistência de uma cópia de $\mathcal{M}$ para $\varepsilon>0$, pequeno. Daqui em diante, assumiremos que $\mathcal{M}$ é uniformemente assintoticamente estável.

Teorema 2.13. Nestas condiçôes, existe $\varepsilon_{0}>0$ tal que para cada $\varepsilon \in\left(0, \varepsilon_{0}\right)$ (2.35) possui uma variedade invariante

$$
\mathcal{M}_{\varepsilon}=\{(x, y): x=\bar{x}(y, \varepsilon), y \in V\} .
$$

Além disso, $\mathcal{M}_{\varepsilon} \cong \mathcal{M}$ e $\bar{x}(y, \varepsilon)=x^{\star}(y)+\mathcal{O}(\varepsilon)$.

A variedade $\mathcal{M}_{\varepsilon}$ é chamada de variedade adiabática. A solução a partir de qualquer condição inicial sobre $\mathcal{M}_{\varepsilon}$ é da forma $(\bar{x}(y(\tau), \varepsilon), y(\tau))$, onde $y(\tau)$ é solução do sistema reduzido

$$
y^{\prime}=g(\bar{x}(y, \varepsilon), y) .
$$


Segue de alguns dos resultados sobre variedades centrais, apresentados em [Car81], o teorema de aproximação 2.14 .

Teorema 2.14. Para cada $\varepsilon \in\left(0, \varepsilon_{0}\right)$, existe uma vizinhança de $\mathcal{M}_{\varepsilon}, \mathcal{N}_{\varepsilon} \subset \mathbb{R}^{n+m}$, tal que para qualquer condiçẫo inicial $\left(x_{0}, y_{0}\right) \in \mathcal{N}_{\varepsilon}$, com solução $(x(\tau), y(\tau))$, existem constantes $K, \alpha>0$ e uma solução do sistema reduzido (2.41), $\hat{y}(\tau)$, tais que

$$
\|(x(\tau), y(\tau))-(\bar{x}(\hat{y}(\tau), \varepsilon), \hat{y}(\tau))\| \leq K\left\|\left(x_{0}, y_{0}\right)-\left(\hat{x}_{0}, \hat{y}_{0}\right)\right\| e^{-\alpha \tau / \varepsilon},
$$

para todo $\tau>0$ tal que $(\hat{y}(\tau), \varepsilon)$ esteja no dominio de $\bar{x}$.

Assim, conseguimos obter informações sobre o sistema (2.35) através da análise do sistema reduzido (2.41), o qual possui menos variáveis. A dificuldade deste processo é determinar a parametrização $\bar{x}(y, \varepsilon)$ da variedade adiabática. Pela propriedade de invariância, esta função deve satisfazer a igualdade

$$
\varepsilon \frac{\partial \bar{x}}{\partial y}(y, \varepsilon) g(\bar{x}(y, \varepsilon), \varepsilon)=f(\bar{x}(y, \varepsilon), \varepsilon),
$$

a qual dificilmente resolvemos explicitamente. No entanto, usando o método de aproximação de variedades centrais de [Car81], conseguimos obter sua expansão em primeira ordem em $\varepsilon$

$$
\bar{x}(y, \varepsilon)=x^{\star}(y)-\varepsilon A^{\star}(y)^{-2} \partial_{y} f\left(x^{\star}(y), y\right) g\left(x^{\star}(y), y\right)+\mathcal{O}\left(\varepsilon^{2}\right) .
$$




\section{Capítulo 3}

\section{Equações de Movimento para Deformação Linear}

Do Capítulo 2, constatamos que a formulação Lagrangiana para problemas envolvendo corpos contínuos (viscoelásticos) é razoável, já que recupera as mesmas equações de movimento deduzidas de outras formas e extensivamente usadas em diversos contextos.

Destacamos duas grandes vantagens da mecânica Lagrangiana com função dissipação: sua invariância por sistemas de coordenadas (equação (1.3)) e seu fácil tratamento ao restringir o movimento a subvariedades (Teorema 2.2). Usando estas duas propriedades, faremos a imposição de vínculos sobre as deformações do corpo elástico de forma a reduzir o sistema a um número finito de graus de liberdade. Esta imposição permitirá tratarmos o problema dinâmico com precisão, evitando cálculos formais. Parte dos resultados apresentados neste capítulo podem ser encontrados em [RR15] e [FMGRR15].

\subsection{Corpos Pseudo-Rígidos}

Tratemos inicialmente do problema de um corpo girante isolado, seguindo as mesmas hipóteses do Capítulo 2. Ou seja, sobre este corpo atuam suas forças elásticas internas e a gravidade. Assumiremos que em repouso ele tenha formato esférico, com densidade de massa radialmente simétrica, $\rho(x)=\rho(\|x\|), \quad \forall x \in \mathcal{B}$, e raio $R$. Assumiremos novamente que o material é Hookeano, ou seja, está sujeito ao potencial (2.26). Além disso, também assumiremos que seja incompressível.

Sendo $\phi(t, x)$ a posição do ponto $x \in \mathcal{B}$ no instante $t$, estabeleçamos o seguinte vínculo:

$$
\phi(t, x):=G(t) x, \quad \forall x \in \mathcal{B},
$$

onde $\operatorname{det} G(t)=+1, \forall t$. Observe que fixamos o centro de massa na origem devido à ausência de forças externas.

Note que dado $G \in \mathrm{SL}(3)$, como acima, $G^{T} G \in \operatorname{SSym}_{+}(3)=\left\{T \in \mathrm{M}(3): T=T^{T}, \quad T>\right.$ $0, \quad \operatorname{det} T=1\}$. Definindo $A=\sqrt{G^{T} G}$, obtemos $G=\left(G A^{-1}\right) A=Y A$. É claro que $Y^{T} Y=$ $A^{-1} G^{T} G A^{-1}=\mathrm{Id}$, logo $Y \in \mathrm{SO}(3)$. Também é claro que esta decomposição é única, pois se $Y_{1} A_{1}=Y_{2} A_{2} \Rightarrow A_{1}^{2}=\left(Y_{1} A_{1}\right)^{T}\left(Y_{1} A_{1}\right)=\left(Y_{2} A_{2}\right)^{T}\left(Y_{2} A_{2}\right)=A_{2}^{2}$, decorrendo que $A_{1}=A_{2}$ por serem positivas definidas (estas definições métricas são Euclidianas).

Portanto, a aplicação

$$
\Phi: \mathrm{SO}(3) \times \operatorname{SSym}_{+}(3) \longrightarrow \mathrm{SL}(3)
$$

$\Phi(Y, A):=Y A$, é bijetora. Esta é a conhecida decomposição polar de uma matriz inversível. 
Proposição 3.1. O conjunto $\operatorname{SSym}_{+}(3) \subset \operatorname{Sym}(3)$ é uma subvariedade conexa de dimensão 5 .

Demonstração. Considere $F: \operatorname{Sym}(3) \longrightarrow \mathbb{R}, F(M)=\operatorname{det} M$. Supondo que $F\left(M_{0}\right)=1$, temos que

$$
D F_{M_{0}} M_{0}=\left.\frac{d}{d \lambda}\right|_{\lambda=1} \operatorname{det}\left(\lambda M_{0}\right)=3 \neq 0
$$

logo 1 é valor regular de $F$, o que garante que $F^{-1}(1)$ é subvariedade mergulhada. Observe agora que $\operatorname{SSym}_{+}(3) \subset F^{-1}(1)$ é aberto (é fácil ver que seu complementar é fechado), portanto também é mergulhado em $\mathrm{M}(3)$. Na verdade se trata de uma componente conexa, pois podemos ligar quaisquer dois de seus pontos, $A_{1}, A_{2} \in \mathrm{SSym}_{+}(3)$, pelo caminho:

$$
\gamma(t):=\frac{1}{\operatorname{det}\left((1-t) A_{1}+t A_{2}\right)^{\frac{1}{3}}}\left((1-t) A_{1}+t A_{2}\right) \in \operatorname{SSym}_{+}(3), \quad \forall t \in[0,1]
$$

Desta forma, concluímos que $T_{A} \operatorname{SSym}_{+}(3) \equiv \operatorname{KerDF} F_{A}=\left\{H \in \operatorname{Sym}(3): \operatorname{Tr}\left(A^{-1} H\right)=0\right\}$.

Proposição 3.2. A função $\Phi$ é um difeomorfismo.

Demonstração. Lembrando que $\Phi$ é bijetora e que as dimensões do domínio e da imagem são iguais, basta verificar que o núcleo de $D \Phi_{(Y, A)}$ é trivial, para todos $Y, A$. Para isto, dado $A_{0} \in \operatorname{SSym}_{+}(3)$ defina a função suave $f_{A_{0}}: \operatorname{SSym}_{+}(3) \longrightarrow \operatorname{SSym}_{+}(3), f_{A_{0}}(A):=\sqrt{A_{0}} A \sqrt{A_{0}}$. Note que $f_{A_{0}}$ é difeomorfismo, com $f_{A_{0}}^{-1}=f_{A_{0}^{-1}}$. Concluímos disso que:

$$
\begin{gathered}
T_{A_{0}} \operatorname{SSym}_{+}(3) \equiv D f_{A_{0}}\left(T_{\text {Id }} \operatorname{SSym}_{+}(3)\right)=\left\{\sqrt{A_{0}} B \sqrt{A_{0}}: B=B^{T}, \operatorname{Tr} B=0\right\}= \\
=\left\{H \in \operatorname{Sym}(3): \operatorname{Tr}\left(A_{0}^{-1} H\right)=0 .\right\}
\end{gathered}
$$

Tome agora uma curva arbitrária $(Y(t), A(t)) \in \mathrm{SO}(3) \times \operatorname{SSym}_{+}(3), \operatorname{com}(Y(0), A(0))=$ $\left(Y_{0}, A_{0}\right)$. Suponha que $D \Phi_{\left(Y_{0}, A_{0}\right)}(\dot{Y}(0), \dot{A}(0))=0$.

$$
\Rightarrow 0=\left.\frac{d}{d t}\right|_{t=0} Y(t) A(t)=Y_{0} \xi A_{0}+Y_{0} \sqrt{A_{0}} B \sqrt{A_{0}},
$$

onde $\xi=Y_{0}^{T} \dot{Y}(0) \in \operatorname{skew}(3)$.

$$
\Rightarrow-\sqrt{A_{0}} B{\sqrt{A_{0}}}^{-1}=\xi=(-\xi)^{T}={\sqrt{A_{0}}}^{-1} B \sqrt{A_{0}} \Rightarrow \operatorname{det} B=-\operatorname{det} B .
$$

Logo, $B \in T_{\text {Id }} \operatorname{SSym}_{+}(3)$ é simétrica, com determinante e traço nulos. Assim, existe $Y \in \mathrm{SO}(3)$ tal que $Y^{T} B Y=\operatorname{diag}(\lambda,-\lambda, 0)$. Sendo assim, da igualdade $B A_{0}=-A_{0} B$, concluímos que:

$$
\begin{aligned}
\left\langle e_{1}, Y^{T} B Y Y^{T} A_{0} Y e_{1}\right\rangle= & -\left\langle e_{1}, Y^{T} A_{0} Y Y^{T} B Y e_{1}\right\rangle \Rightarrow\left\langle Y^{T} B Y e_{1}, Y^{T} A_{0} Y e_{1}\right\rangle=-\lambda\left\langle e_{1}, Y^{T} A_{0} Y e_{1}\right\rangle \\
& \Rightarrow \lambda\left\langle Y e_{1}, A_{0} Y e_{1}\right\rangle=-\lambda\left\langle Y e_{1}, A_{0} Y e_{1}\right\rangle \Rightarrow \lambda=0,
\end{aligned}
$$

onde a última implicação segue de $Y e_{1} \neq 0$ e da hipótese de $A_{0}$ ser positiva. Portanto $B=0, \xi=$ $0 \Rightarrow(\dot{Y}(0), \dot{A}(0))=(0,0)$.

Assim, o espaço de configurações desse corpo será $\mathrm{SO}(3) \times \operatorname{SSym}_{+}(3)$. À matriz $Y$ associamos a rotação da matéria, e $A$ a deformação do sólido no referencial do corpo.

O vínculo (3.1) de certa forma foi introduzido desde os trabalhos de Dirichlet. Este modelo atualmente é denominado como corpo pseudo-rígido e é aplicado em diversas situações (consultar referências de [RSD99]). Uma das abordagens mais recentes a este assunto encontra-se em 
$\left[\mathrm{KVG}^{+} 12\right]$, onde é estudado um corpo pseudo-rígido em interação com um corpo pontual. O tratamento se dá com o formalismo de Geometria Simplética, sendo possível fazer reduções do sistema e eliminar as simetrias presentes. Um de nossos objetivos é entender a influência da viscosidade sobre o movimento, o que geralmente impede o uso da formulação Hamiltoniana da mecânica, como visto na Introdução.

A primeira pergunta que surge é o quão drástica seria nossa hipótese (3.1). A informação perdida nesta passagem é desprezível o suficiente para conservarmos as principais propriedades dinâmicas do problema original?

Há pelo menos dois trabalhos, [Mun84a] e [Mun84b], onde se estuda esta relação em um contexto matemático. Na primeira, é feita uma teoria abstrata baseada no conceito de variedades invariantes buscando exibir um método de extrair teorias grosseiras (ou inferiores) a partir de teorias finas (completas). Citam-se como exemplos de teorias inferiores à teoria de sólidos, a mecânica de partículas, a mecânica de corpos rígidos e a teoria de hastes; onde em cada uma dessas é feita uma hipótese sobre o sistema (pequenas dimensões dos corpos, rigidez e corpo alongado). Já o segundo artigo é dedicado ao problema concreto do reconhecimento do corpo pseudo-rígido como teoria inferior associada à teoria de sólidos.

Em [NO84], são discutidas as semelhanças entre esse modelo do corpo pseudo-rígido e a teoria de ponto de Cosserat, alternativamente usada para descrever o movimento de sólidos com poucos graus de liberdade.

O movimento deste corpo pode ser obtido simplesmente através das equações de Euler-Lagrange sobre SL(3). Porém por analogia ao corpo rígido, é preciso entender com que velocidade a matéria gira no espaço. Uma alternativa é encontrar um referencial que se mova juntamente com o objeto, o que é imediato para o corpo rígido, mas necessita de argumentos caso haja deformação.

A escolha usual, que remete ao próprio Dirichlet, é tomar a decomposição singular da matriz $G$,

$$
G=R \tilde{A} S^{T}, \quad R, S \in \mathrm{SO}(3), \quad \tilde{A}=\operatorname{diag}\left(a_{1}, a_{2}, a_{3}\right)>0 .
$$

Tal decomposição pode ser obtida através da decomposição polar de $G$ e em seguida diagonalizando a matriz simétrica $A$ (por matriz de rotação). À matriz $R$ é associada a rotação do corpo, a $S^{T}$ a rotação do fluido em seu interior e a $\tilde{A}$ a mudança de seu formato, como indica a Figura 3.1, extraída de $\left[\mathrm{KVG}^{+} 12\right]$.
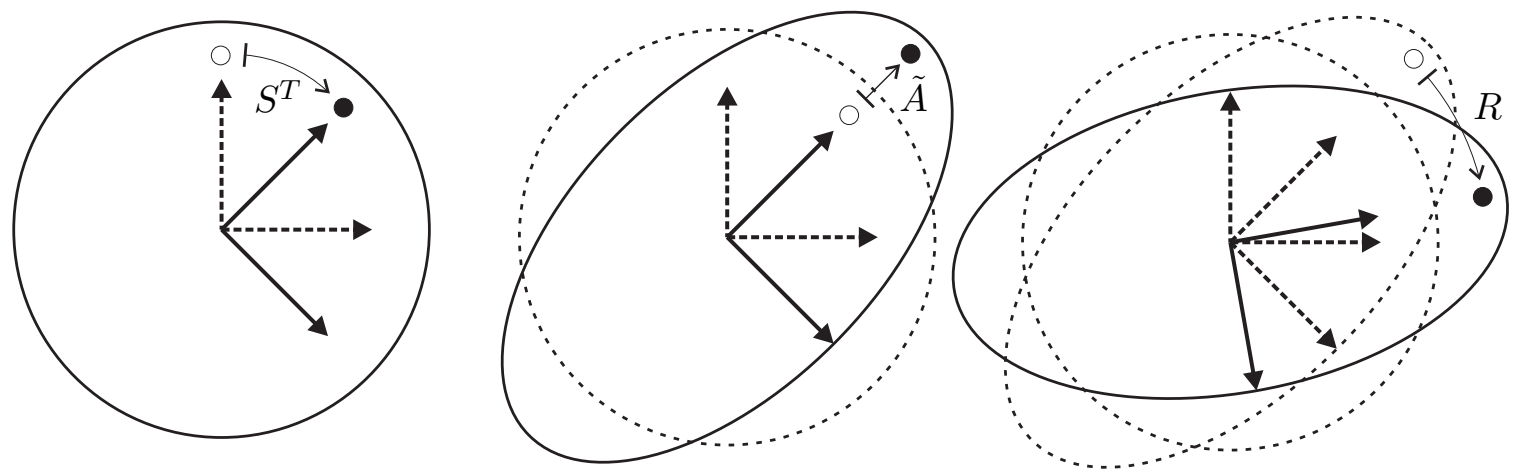

Figura 3.1: Operações envolvidas na decomposição singular.

No entanto, claramente esta decomposição singular não é única. Mais do que isto, dada uma curva analítica $G(t) \in \mathrm{SL}(3)$, prova-se que existem $R(t), \tilde{A}(t), S(t)$ analíticas tais que $G(t)=$ $R(t) \tilde{A}(t) S^{T}(t)$, porém existe curva $\mathcal{C}^{\infty}, G(t) \in \mathrm{SL}(3)$, que não admite a mesma decomposição em matrizes $\mathcal{C}^{\infty}$ (prova em [FL01]). A Proposição 3.2 garante que isto não é possível para a decomposição polar.

Além disso, apesar de o operador $R$ representar a rotação da "forma" do corpo, não representa a rotação da matéria, o que fica mais claro nos casos de baixa excentricidade em que estamos 

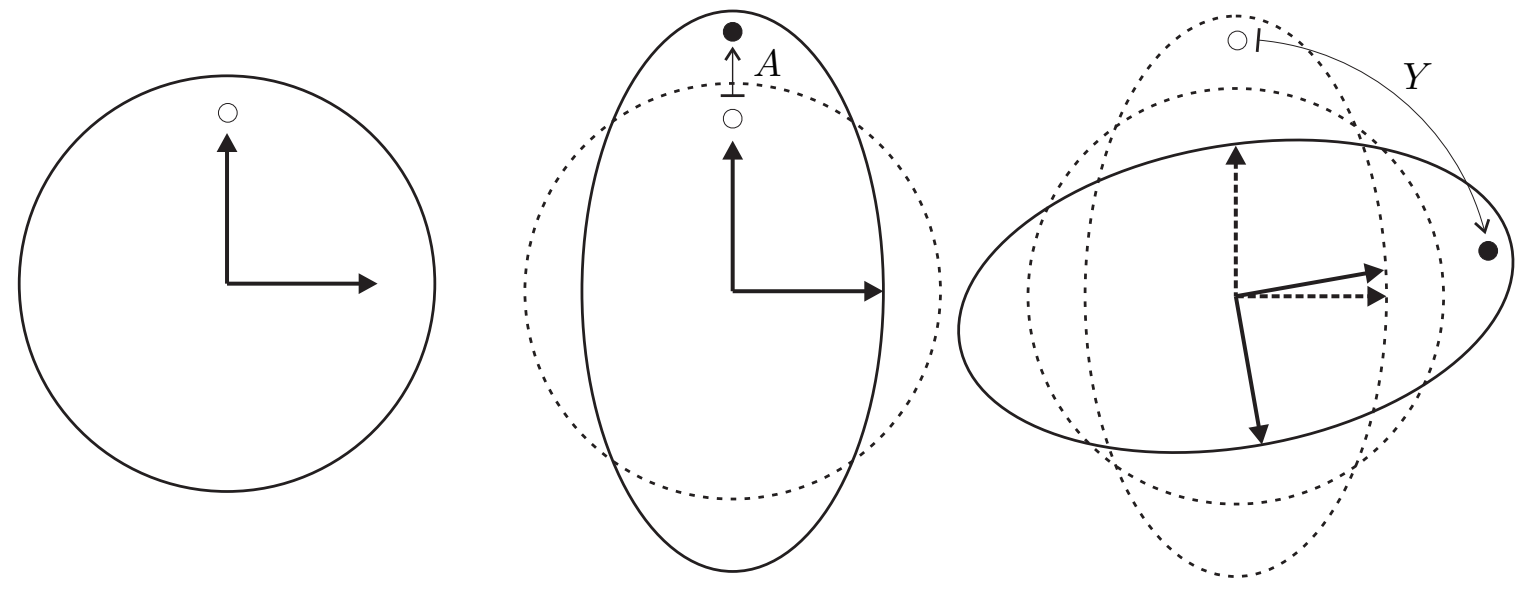

Figura 3.2: Operações envolvidas na decomposição polar.

interessados. Por exemplo, dado $\varepsilon>0$ pequeno, tome

$$
\tilde{A}_{\varepsilon}=\left(\begin{array}{ccc}
a+\varepsilon & 0 & 0 \\
0 & a-\varepsilon & 0 \\
0 & 0 & \left(a^{2}-\varepsilon^{2}\right)^{-1}
\end{array}\right) \quad R(t), S(t)=\left(\begin{array}{ccc}
\cos \left(\omega_{j} t\right) & -\operatorname{sen}\left(\omega_{j} t\right) & 0 \\
\operatorname{sen}\left(\omega_{j} t\right) & \cos \left(\omega_{j} t\right) & 0 \\
0 & 0 & 1
\end{array}\right)
$$

onde, na definição de $R$ usamos $j=1$ e na definição de $S, j=2$. Assim,

$$
\begin{gathered}
G_{\varepsilon}=\left(\begin{array}{ccc}
\cos \left(\left(\omega_{1}-\omega_{2}\right) t\right) & -\operatorname{sen}\left(\left(\omega_{1}-\omega_{2}\right) t\right) & 0 \\
\operatorname{sen}\left(\left(\omega_{1}-\omega_{2}\right) t\right) & \cos \left(\left(\omega_{1}-\omega_{2}\right) t\right) & 0 \\
0 & 0 & 1
\end{array}\right)\left(\begin{array}{ccc}
a & 0 & 0 \\
0 & a & 0 \\
0 & 0 & \left(a^{2}-\varepsilon^{2}\right)^{-1}
\end{array}\right) \\
+\varepsilon\left(\begin{array}{ccc}
\cos \left(\left(\omega_{1}+\omega_{2}\right) t\right) & \operatorname{sen}\left(\left(\omega_{1}+\omega_{2}\right) t\right) & 0 \\
\operatorname{sen}\left(\left(\omega_{1}+\omega_{2}\right) t\right) & -\cos \left(\left(\omega_{1}+\omega_{2}\right) t\right) & 0 \\
0 & 0 & 0
\end{array}\right) .
\end{gathered}
$$

Portanto, a parte relevante do movimento é apenas uma rotação em torno do eixo $z$ com velocidade angular dada por $\omega_{1}-\omega_{2}$. Note que esta primeira matriz está decomposta na forma polar. Um caso drástico ocorre quando $\varepsilon=0$ e $\omega_{1}=\omega_{2}$. Notamos que $\phi(t, x)=\tilde{A} x$, independe do tempo e até mesmo da velocidade angular. Assim, já que estaremos interessados em pequenas deformações $(A \approx \mathrm{Id})$ e nos efeitos da viscosidade, a qual depende do movimento relativo entre as partes do corpo, sem dúvida devemos partir da decomposição polar. Veja sua ilustração na Figura 3.2.

Neste caso, a energia cinética fica

$$
\begin{aligned}
T=\frac{1}{2} \int_{\mathcal{B}} \rho(\|x\|)\left\|\frac{\partial}{\partial t} Y A x\right\|^{2} d x=\frac{1}{2} \int_{\mathcal{B}} \rho(\|x\|)\|\dot{Y} A x+Y \dot{A} x\|^{2} d x \\
=\frac{1}{2} \int_{\mathcal{B}} \rho(\|x\|)\|\Omega A x+\dot{A} x\|^{2} d x=\frac{1}{2} \int_{\mathcal{B}} \rho(\|x\|)\left(\|\Omega A x\|^{2}+\|\dot{A} x\|^{2}+2\langle x, \dot{A} \Omega A x\rangle\right) d x
\end{aligned}
$$

onde $\Omega=Y^{T} \dot{Y}$ novamente é a velocidade angular no referencial do corpo.

Note que, para todo $C \in \mathrm{M}(3)$, sendo $\mathcal{B}$ a bola de raio $R$ e $x=\left(x_{1}, x_{2}, x_{3}\right)$,

$$
\int_{\mathcal{B}} \rho(\|x\|)\langle x, C x\rangle d x=\int_{\mathcal{B}} \rho(\|x\|)\left(x_{1}^{2}+x_{2}^{2}\right) d x \cdot \frac{\operatorname{Tr}(C)}{2}=\frac{\mathrm{I}_{\circ}}{2} \operatorname{Tr}(C),
$$

onde $\mathrm{I}_{\circ}$ é o momento de inércia da bola em relação a um eixo que passa pela origem. Para o caso homogêneo, lembramos que $\mathrm{I}_{\circ}=2 M R^{2} / 5$. 
Assim, a energia cinética fica

$$
T=\frac{\mathrm{I}_{\circ}}{4}\left(\operatorname{Tr}\left(\dot{A}^{2}\right)-\operatorname{Tr}\left(\Omega^{2} A^{2}\right)+2 \operatorname{Tr}(\dot{A} \Omega A)\right) .
$$

Voltemos à energia potencial elástica (2.26). Em [LL86] vemos que ela é em princípio definida através do tensor de deformações

$$
\mathcal{E}=\frac{1}{2}\left(d u+d u^{T}+d u^{T} d u\right)
$$

por

$$
V_{e l}=\int_{\mathcal{B}} \mu \operatorname{Tr}\left(\mathcal{E}^{2}\right)+\left(\frac{K}{2}-\frac{\mu}{3}\right) \operatorname{Tr}(\mathcal{E})^{2} d x
$$

sendo $\mu$ é o módulo de cisalhamento, $K$ o módulo volumétrico (adiabáticos) e $u(t, x)=\phi(t, x)-x$.

Respeitado o vínculo (3.1), temos

$$
\mathcal{E}=\frac{1}{2}\left(A^{2}-\mathrm{Id}\right) \approx A-\mathrm{Id},
$$

supondo $A \approx \operatorname{Id}$ (esta aproximação ficará clara na Seção 3.2). Observe que este tensor consistentemente não depende da rotação do corpo.

Portanto, o vínculo impõe a energia potencial

$$
V_{e l}(A)=\frac{4 \pi R^{3}}{3}\left(\mu \operatorname{Tr}\left(A^{2}\right)+\left(\frac{K}{2}-\frac{\mu}{3}\right) \operatorname{Tr}(A)^{2}+\frac{3 K}{2}(3-2 \operatorname{Tr}(A))\right) .
$$

A Proposição 3.3 ressalta que as forças geradas por este potencial atuam no corpo de forma a restaurar seu formato à posição relaxada, analogamente a uma mola.

Proposição 3.3. Tome quaisquer valores de $\mu, K \geq 0$ (não ambos nulos) e $A \in \operatorname{SSym}_{+}(3)$. Temos as seguintes propriedades:

(i) $\operatorname{Tr}(A)^{2} \leq 3 \operatorname{Tr}\left(A^{2}\right)$;

(ii) $\operatorname{Tr}(A) \geq 3$;

(iii) $V_{e l}(A) \geq 0$.

Sendo que as igualdades são satisfeitas se, e somente se, $A=\mathrm{Id}$.

Demonstração. Como $\left\langle H_{1}, H_{2}\right\rangle:=\operatorname{Tr}\left(H_{1}^{T} H_{2}\right)$ define um produto interno, pela desigualdade triangular temos que $\operatorname{Tr}(A)=\langle A, \mathrm{Id}\rangle \leq\|A\|\|\operatorname{Id}\|=\sqrt{3} \sqrt{\operatorname{Tr}\left(A^{2}\right)}$. A igualdade é atingida se, e só se, $A=\lambda \mathrm{Id}$, ou seja, $A=\mathrm{Id}$.

Sejam $x, c>0$ tais que $x^{-1}, c^{-1}, x c$ sejam os autovalores de $A$. Sendo $f_{c}(x):=c x+x^{-1}+c^{-1}$, $f_{c}^{\prime}(x)=c x^{-2}\left(x^{2}-c^{-1}\right)$. Logo, $f_{c}(x)$, para $x>0$, possui mínimo global em $x=c^{-\frac{1}{2}}$. Ou seja, $f_{c}(x) \geq f_{c}\left(c^{-\frac{1}{2}}\right)=h(c):=2 c^{\frac{1}{2}}+c^{-1} \forall x, c>0$. Mas, $h^{\prime}(c)=c^{-2}\left(c^{\frac{3}{2}}-1\right)$, portanto $h(c) \geq h(1)=3$. Assim, $\operatorname{Tr}(A)=f_{c}(x) \geq h(c) \geq 3$, e devido aos mínimos serem estritos, a igualdade só é atingida na identidade.

A terceira afirmação é consequência imediata das duas primeiras e da expressão para $V_{e l}$ :

$$
V_{e l}(A)=\frac{4 \pi R^{3}}{3}\left(\mu\left(\operatorname{Tr}\left(A^{2}\right)-\frac{1}{3} \operatorname{Tr}(A)^{2}\right)+\frac{K}{2}(\operatorname{Tr}(A)-3)^{2}\right) .
$$

Pode-se ainda adicionar o potencial gravitacional do corpo sobre ele próprio. De (2.30), este potencial adquire a forma

$$
V_{s g}(A)=-\frac{G}{2} \int_{\mathcal{B}} \int_{\mathcal{B}} \frac{\rho(x) \rho(y)}{\|A(x-y)\|} d x d y
$$


É fácil ver (desde que $A>0$ ) que esta integral é convergente, e diferenciável. Daqui em diante, até o final desta Seção 3.1, assumiremos que o corpo tem distribuição de massa homogênea.

Proposição 3.4. Para um corpo homogêneo, vale a seguinte identidade

$$
V_{s g}(A)=-\frac{3 M^{2} G}{10 R} \int_{0}^{\infty} \frac{1}{\sqrt{\operatorname{det}\left(A^{2}+\lambda \mathrm{Id}\right)}} d \lambda, \quad \forall A \in \operatorname{SSym}_{+}(3) .
$$

Observação 3.5. Esta expressão é utilizada desde Dirichlet. Para uma prova, consultar Teorema 3, parágrafo 18 de [Cha87], ou o artigo 494 de [TT09].

A Proposição 3.6 garante que este potencial é sempre limitado inferiormente e superiormente.

Proposição 3.6. Para todo $A \in \operatorname{SSym}_{+}(3)$,

$$
-\frac{3 M^{2} G}{5 R} \leq V_{s g}(A)<0 .
$$

A igualdade é atingida somente se $A=\mathrm{Id}$.

Demonstração. Sejam $a_{1}, a_{2}, a_{3}>0$ os autovalores de $A$. Assim, $\operatorname{det}\left(A^{2}+\lambda \mathrm{Id}\right)=\lambda^{3}+\left(a_{1}^{2}+\right.$ $\left.a_{2}^{2}+a_{3}^{2}\right) \lambda^{2}+\left(a_{1}^{2} a_{2}^{2}+a_{1}^{2} a_{3}^{2}+a_{2}^{2} a_{3}^{2}\right) \lambda+\left(a_{1} a_{2} a_{3}\right)^{2}=\lambda^{3}+\operatorname{Tr}\left(A^{2}\right) \lambda^{2}+\operatorname{Tr}(\tilde{A}) \lambda+1$, onde $\tilde{A}=$ $\operatorname{diag}\left(a_{1}^{2} a_{2}^{2}, a_{1}^{2} a_{3}^{2}, a_{2}^{2} a_{3}^{2}\right) \in \operatorname{SSym}_{+}(3)$. Portanto, do item (ii) da Proposição 3.3 , decorre que $\operatorname{det}\left(A^{2}+\right.$ $\lambda$ Id) $\geq \lambda^{3}+3 \lambda^{2}+3 \lambda+1=(\lambda+1)^{3}$. Assim,

$$
V_{s g}(A) \geq-\frac{3 M^{2} G}{10 R} \int_{0}^{\infty}(\lambda+1)^{-\frac{3}{2}} d \lambda=-\frac{3 M^{2} G}{5 R} .
$$

A deformação terá também componente viscosa, linear. Sua contribuição será calculada através da função de Rayleigh, tradicionalmente ([LL86], [BL87] e [Efr00]) introduzida em (2.27). Uma definição equivalente àquela apresentada em (2.27) é dada ao substituir o tensor de deformações pelo tensor $\dot{\mathcal{E}}=\dot{A}$ em (3.5).

Assim,

$$
\mathcal{D}=\int_{\mathcal{B}} \eta \operatorname{Tr}\left(\dot{\mathcal{E}}^{2}\right)+\left(\frac{\zeta}{2}-\frac{\eta}{3}\right) \operatorname{Tr}(\dot{\mathcal{E}})^{2} d x=\frac{4 \pi R^{3}}{3}\left(\eta \operatorname{Tr}\left(\dot{A}^{2}\right)+\left(\frac{\zeta}{2}-\frac{\eta}{3}\right) \operatorname{Tr}(\dot{A})^{2}\right) .
$$

Proposição 3.7. Tome quaisquer valores de $\eta>0, \zeta \geq 0$ e $\dot{A} \in T_{A} \operatorname{SSym}_{+}$(3). Decorre que $\mathcal{D}(\dot{A}) \geq 0$, e a igualdade é atingida se e somente se $\dot{A}=0$.

Demonstração. Novamente, note que

$$
\mathcal{D}(\dot{A})=\frac{4 \pi R^{3}}{3}\left(\eta\left(\operatorname{Tr}\left(\dot{A}^{2}\right)-\frac{1}{3} \operatorname{Tr}(\dot{A})^{2}\right)+\frac{\zeta}{2} \operatorname{Tr}(\dot{A})^{2}\right) .
$$

O raciocínio na prova do item (i) da Proposição 3.3 se aplica aqui, garantindo que $\mathcal{D} \geq 0$. Concluímos que se $\mathcal{D}(\dot{A})=0$, então $\dot{A}=\lambda$ Id. Mas, $0=\operatorname{Tr}\left(A^{-1} \dot{A}\right)=\lambda \operatorname{Tr}\left(A^{-1}\right)$. Logo, $\dot{A}=0$.

Para calcular as equações de movimento, também usaremos o Teorema dos Multiplicadores de Lagrange na forma (2.5). Em $\operatorname{SSym}_{+}(3) \subset \mathrm{M}(3) \equiv \mathbb{R}^{9}$, usemos novamente a métrica Euclidiana, $<H_{1}, H_{2}>:=\operatorname{Tr}\left(H_{1}^{T} H_{2}\right)$.

Proposição 3.8. A projeção ortogonal para este caso particular é, $P_{A}: \mathrm{M}(3) \longrightarrow T_{A} \mathrm{SSym}_{+}(3)$

$$
P_{A}(H)=\frac{1}{2}\left(H+H^{T}\right)-\frac{\operatorname{Tr}\left(A^{-1} H\right)}{\operatorname{Tr}\left(A^{-2}\right)} A^{-1}, \quad \forall H \in \mathrm{M}(3) .
$$


Demonstração. Note que, para todo $H \in \mathrm{M}(3), P_{A}(H) \in \operatorname{Sym}(3)$ e $\operatorname{Tr}\left(A^{-1} P_{A}(H)\right)=0$, além de ser linear. Assim, de (3.2), vemos que $P_{A}$ está bem definida. Além disso, se $H \in T_{A} \operatorname{SSym}_{+}(3)$, $P_{A}(H)=H$. Portanto, $P_{A}$ é sobrejetora e $P_{A}^{2}=P_{A}$. Falta verificar que seu núcleo é ortogonal a sua imagem. Mas, isto decorre da seguinte equação, $\forall H_{1}, H_{2}$ :

$\left\langle P_{A}\left(H_{1}\right), H_{2}\right\rangle=\frac{1}{2}\left(\operatorname{Tr}\left(H_{1} H_{2}\right)+\operatorname{Tr}\left(H_{1}^{T} H_{2}\right)\right)-\frac{1}{\operatorname{Tr}\left(A^{-2}\right)} \operatorname{Tr}\left(A^{-1} H_{1}\right) \operatorname{Tr}\left(A^{-1} H_{2}\right)=\left\langle H_{1}, P_{A}\left(H_{2}\right)\right\rangle$.

Através de um cálculo simples, obtemos as seguintes equações de movimento

$$
P_{A}\left[\frac{d}{d t}\left(\frac{\partial \mathcal{L}}{\partial \dot{A}}\right)-\frac{\partial \mathcal{L}}{\partial A}+\frac{\partial \mathcal{D}}{\partial \dot{A}}\right]=0 .
$$

Assim,

$$
\begin{gathered}
\frac{M R^{2}}{5}\left(\ddot{A}+\frac{1}{2}[\dot{\Omega}, A]+[\Omega, \dot{A}]+\frac{1}{2}\left(\Omega^{2} A+A \Omega^{2}\right)\right) \\
\left.+\frac{4 \pi R^{3}}{3}\left(2 \mu A+\left(K-\frac{2 \mu}{3}\right) \operatorname{Tr}(A) \operatorname{Id}-3 K \operatorname{Id}\right)+\frac{4 \pi R^{3}}{3}\left(2 \eta \dot{A}+\left(\zeta-\frac{2 \eta}{3}\right) \operatorname{Tr}(\dot{A}) \operatorname{Id}\right)\right) \\
=\frac{1}{\operatorname{Tr}\left(A^{-2}\right)}\left(\frac{M R^{2}}{5}\left(\operatorname{Tr}\left(A^{-1} \ddot{A}\right)+2 \operatorname{Tr}\left(A^{-1} \Omega \dot{A}\right)+\operatorname{Tr}\left(\Omega^{2}\right)\right)\right. \\
+\frac{4 \pi R^{3}}{3}\left(6 \mu+\left(K-\frac{2 \mu}{3}\right) \operatorname{Tr}(A) \operatorname{Tr}\left(A^{-1}\right)-3 K \operatorname{Tr}\left(A^{-1}\right)\right) \\
\left.+\frac{4 \pi R^{3}}{3}\left(2 \eta \operatorname{Tr}\left(A^{-1} \dot{A}\right)+\left(\zeta-\frac{2 \eta}{3}\right) \operatorname{Tr}(\dot{A}) \operatorname{Tr}\left(A^{-1}\right)\right)\right) A^{-1} \\
-\frac{3 M^{2} G}{10 R}\left(\int_{0}^{\infty} \frac{A\left(A^{2}+\lambda \operatorname{Id}\right)^{-1}}{\sqrt{\operatorname{det}\left(A^{2}+\lambda \mathrm{Id}\right)}} d \lambda-\frac{1}{\operatorname{Tr}\left(A^{-2}\right)}\left(\int_{0}^{\infty} \frac{\operatorname{Tr}\left(\left(A^{2}+\lambda \mathrm{Id}\right)^{-1}\right)}{\sqrt{\operatorname{det}\left(A^{2}+\lambda \mathrm{Id}\right)}} d \lambda\right) A^{-1}\right) .
\end{gathered}
$$

Assumamos a existência de solução da equação em $\operatorname{SSym}_{+}(3)$. Neste caso, a solução $A(t)$ também deve satisfazer à equação acima. Mas, $A^{-1} A=\mathrm{Id}$ implica

$$
\begin{gathered}
\frac{d}{d t}\left(A^{-1}\right) A=-A^{-1} \dot{A}, \\
0=\frac{d}{d t} \operatorname{det}(A)=D \operatorname{det}_{A}(\dot{A})=\left.\frac{d}{d \lambda}\right|_{\lambda=0} \operatorname{det}(A+\lambda \dot{A})=\left.\operatorname{det}(A) \frac{d}{d \lambda}\right|_{\lambda=0} \operatorname{det}\left(\operatorname{Id}+\lambda A^{-1} \dot{A}\right)=\operatorname{Tr}\left(A^{-1} \dot{A}\right) \\
\Rightarrow 0=\operatorname{Tr}\left(\frac{d}{d t} A^{-1} \dot{A}\right)+\operatorname{Tr}\left(A^{-1} \ddot{A}\right) \Rightarrow \operatorname{Tr}\left(A^{-1} \ddot{A}\right)=\operatorname{Tr}\left(A^{-1} \dot{A} A^{-1} \dot{A}\right) .
\end{gathered}
$$

Portanto, a curva $A(t)$ deve satisfazer a equação diferencial ordinária, agora escrita na forma explícita

$$
\begin{aligned}
\ddot{A}+ & \frac{1}{2}[\dot{\Omega}, A]+[\Omega, \dot{A}]+\frac{1}{2}\left(\Omega^{2} A+A \Omega^{2}\right)+\frac{20 \pi R}{3 M}\left[2 \mu A+\left(K-\frac{2 \mu}{3}\right) \operatorname{Tr}(A) \operatorname{Id}\right. \\
& \left.-3 K \operatorname{Id}+2 \eta \dot{A}+\left(\zeta-\frac{2 \eta}{3}\right) \operatorname{Tr}(\dot{A}) \operatorname{Id}\right]+\frac{3 M G}{2 R^{3}} \int_{0}^{\infty} \frac{A\left(A^{2}+\lambda \operatorname{Id}\right)^{-1}}{\sqrt{\operatorname{det}\left(A^{2}+\lambda \operatorname{Id}\right)}} d \lambda+\chi A^{-1}=0
\end{aligned}
$$


onde

$$
\begin{gathered}
\chi=\frac{1}{\operatorname{Tr}\left(A^{-2}\right)}\left\{2 \operatorname{Tr}\left(A^{-1} \dot{A} \Omega\right)-\operatorname{Tr}\left(A^{-1} \dot{A} A^{-1} \dot{A}\right)-\operatorname{Tr}\left(\Omega^{2}\right)-\frac{20 \pi R}{3 M}\left[6 \mu+\left(K-\frac{2 \mu}{3}\right) \operatorname{Tr}(A) \operatorname{Tr}\left(A^{-1}\right)\right.\right. \\
\left.\left.-3 K \operatorname{Tr}\left(A^{-1}\right)+\left(\zeta-\frac{2 \eta}{3}\right) \operatorname{Tr}(\dot{A}) \operatorname{Tr}\left(A^{-1}\right)\right]-\frac{3 M G}{2 R^{3}} \int_{0}^{\infty} \frac{\operatorname{Tr}\left(\left(A^{2}+\lambda \mathrm{Id}\right)^{-1}\right)}{\sqrt{\operatorname{det}\left(A^{2}+\lambda \mathrm{Id}\right)}} d \lambda\right\},
\end{gathered}
$$

com a imposição sobre a condição inicial: $\operatorname{Tr}\left(A_{0}^{-1} \dot{A}_{0}\right)=0$.

É simples constatar diretamente pela equação que, de fato, se $A_{0} \in \operatorname{SSym}_{+}(3)$ e $\Omega_{0} \in \operatorname{skew}(3)$ então, $A(t) \in \mathrm{SSym}_{+}(3)$ e $\Omega(t) \in$ skew(3), para todo $t$. Além disso, também pode-se considerar este sistema como de segunda ordem em relação a $Y \in \mathrm{SO}(3)$, adicionando a equação $\dot{Y}=Y \Omega$.

Para calcularmos as equações de Euler-Lagrange para a variável $Y$, usaremos novamente o Lema 2.6 .

Naquela notação,

$$
\frac{\partial \mathcal{L}}{\partial \Omega}=\frac{M R^{2}}{10}\left(A^{2} \Omega+\Omega A^{2}+2 \dot{A} A\right) \Rightarrow W=\frac{M R^{2}}{10}\left(A^{2} \Omega+\Omega A^{2}+\dot{A} A-A \dot{A}\right) .
$$

Assim, a equação fica

$$
\frac{d W}{d t}(t)+[\Omega, W(t)]=0 .
$$

Procedendo com os cálculos, chega-se à equação:

$$
A^{2} \dot{\Omega}+\dot{\Omega} A^{2}+2 \Omega \dot{A} A+2 A \dot{A} \Omega+\left[\Omega^{2}, A^{2}\right]=[A, \ddot{A}] .
$$

Podemos usar a equação (3.7) e substituir $\ddot{A}$ em (3.8)

$$
A^{2} \dot{\Omega}+\dot{\Omega} A^{2}+2 A \dot{\Omega} A+2 \Omega \dot{A} A+2 A \dot{A} \Omega+2 A \Omega \dot{A}+2 \dot{A} \Omega A+\left[\Omega^{2}, A^{2}\right]+\frac{40 \pi R \eta}{3 M}[A, \dot{A}]=0
$$

Note que esta equação não está escrita na forma explícita. Porém, de acordo com o próximo resultado, sempre podemos inverter o operador atuando sobre $\dot{\Omega}$, o isolando, para então substituí-lo na equação (3.7). Obtemos assim o campo vetorial que define a equação diferencial.

Lema 3.9. Para todo $A \in \operatorname{SSym}_{+}(3)$, o operador $\Psi_{A}: \operatorname{skew}(3) \longrightarrow \operatorname{skew}(3), \Psi_{A}(M):=A^{2} M+$ $M A^{2}+2 A M A$, é inversível.

Demonstração. Basta verificar que seu núcleo é trivial. Seja $M \in \operatorname{skew}(3)$ tal que $A^{2} M+M A^{2}+$ $2 A M A=0$. Tomando coordenadas nas quais $A=\operatorname{diag}\left(a_{1}, a_{2}, a_{3}\right)$,

$$
M=\left(\begin{array}{ccc}
0 & -m_{3} & m_{2} \\
m_{3} & 0 & -m_{1} \\
-m_{2} & m_{1} & 0
\end{array}\right)
$$

vemos que esta equação é equivalente a:

$$
\left(\begin{array}{ccc}
0 & -\left(a_{1}+a_{2}\right)^{2} m_{3} & \left(a_{1}+a_{3}\right)^{2} m_{2} \\
\left(a_{1}+a_{2}\right)^{2} m_{3} & 0 & -\left(a_{2}+a_{3}\right)^{2} m_{1} \\
-\left(a_{1}+a_{3}\right)^{2} m_{2} & \left(a_{2}+a_{3}\right)^{2} m_{1} & 0
\end{array}\right)=0 .
$$

Logo, $M=0$.

Note que agora conseguimos um sistema de equações diferenciais escrito na forma explícita. Portanto, podemos usar os resultados padrões da teoria das equações diferenciais ordinárias.

Defina a energia total do sistema como $E(A, \dot{A}, \Omega):=T+V_{e l}+V_{s g}+3 M^{2} G / R$. Decorre das proposições anteriores que $E \geq 0$, e a igualdade é atingida somente quando $\dot{A}=0, A=\mathrm{Id}, \Omega=0$. 
Além disso, da equação (2.7) e da Proposição 3.7, segue que $\dot{E}=-2 \mathcal{D} \leq 0$, e $E(t)$ é constante se, e só se, $\dot{A} \equiv 0$.

Proposição 3.10. O sistema admite a seguinte quantidade conservada, o momento angular no referencial inercial

$$
L(\Omega, A, \dot{A}):=\frac{1}{2} Y\left(A^{2} \Omega+\Omega A^{2}+[\dot{A}, A]\right) Y^{T}=\frac{5}{M R^{2}} Y W Y^{T} .
$$

Demonstração. Considere a ação $\Phi: \mathrm{SO}(3) \times\left(\mathrm{SO}(3) \times \operatorname{SSym}_{+}(3)\right) \longrightarrow \mathrm{SO}(3) \times \operatorname{SSym}_{+}(3)$, $\Phi(R, Y, A):=(R Y, A)$. Para todo $\xi \in$ skew $(3)$, temos que

$$
\mathcal{L}\left(d \Phi_{e^{s \xi}}((Y, A),(\dot{Y}, \dot{A}))\right)=\mathcal{L}\left(\left(e^{s \xi} Y, A\right),\left(e^{s \xi} \dot{Y}, \dot{A}\right)\right)=\mathcal{L}((Y, A),(\dot{Y}, \dot{A})) .
$$

E como $\mathcal{D}$ não depende de $Y, \dot{Y}$, a segunda condição de (2.9) é satisfeita. Logo, pelo Teorema 2.3,

$$
\operatorname{Tr}\left(\left(Y A^{2} \Omega Y^{T}+Y \Omega A^{2} Y^{T}-2 Y A \dot{A} Y^{T}\right) \xi^{T}\right)
$$

é integral primeira, para todo $\xi \in \operatorname{skew}(3)$. Portanto, a parte antissimétrica desta matriz é conservada.

Observação 3.11. Note que a equação (3.8) expressa exatamente esta conservação. É fato elementar que esta quantidade é um múltiplo do momento angular do sistema, $L:=\int_{\mathcal{B}} \rho \vec{r} \times \vec{v} d x$, escrito em forma de matriz antissimétrica, sendo $\vec{r}(x)=Y A x$. No entanto, para o sistema de equações reduzido, (3.7) e (3.9), conseguimos obter somente uma integral primeira, o quadrado do módulo do momento angular, pois este não depende da matriz $Y$. Outro fato que observamos deste resultado é que, apesar da perda de energia, uma solução com condição inicial, de momento angular não nulo, não pode se aproximar do equilíbrio $(\dot{Y}, \dot{A})=(0,0)$ do sistema original. Somente pode atingir equilíbrios relativos $\dot{\Omega}=0$.

Considere agora a ação $\Phi(R, Y, A):=\left(Y R^{T}, R A R^{T}\right)$. Note que da mesma forma,

$$
\mathcal{L}\left(d \Phi_{e^{s \xi}}((Y, A),(\dot{Y}, \dot{A}))\right)=\mathcal{L}\left(\left(Y e^{-s \xi}, e^{s \xi} A e^{-s \xi}\right),\left(\dot{Y} e^{-s \xi}, e^{s \xi} \dot{A} e^{-s \xi}\right)\right)=\mathcal{L}((Y, A),(\dot{Y}, \dot{A})) .
$$

No entanto,

$$
\partial_{\dot{x}} \mathcal{D}\left(\left.\frac{\partial}{\partial s}\right|_{s=0} \Phi_{e^{s \xi}}(x)\right)=\frac{16 \pi R^{3} \eta}{3} \operatorname{Tr}(\dot{A} \xi A),
$$

o qual é diferente de zero, em geral.

No entanto, para o caso conservativo $(\mathcal{D} \equiv 0)$, tal simetria induz uma quantidade conservada. Pelo teorema de Noether,

$$
h=\partial_{\dot{x}} \mathcal{L}(Y, A, \dot{Y}, \dot{A})[(-Y \xi, \xi A-A \xi)]=\operatorname{Tr}\left(\xi^{T}(A \dot{A}+A \Omega A)\right)
$$

é conservada, $\forall \xi \in$ skew(3) (desprezando constantes multiplicativas). Logo, $\Sigma:=[A, \dot{A}]+2 A \Omega A$ é conservada. Esta é a mesma integral primeira expressa na equação (29) de [BKM09], a qual é denominada vorticidade. Já em [Cha87], esta quantidade é denominada circulação.

Note que a vorticidade de um fluido é definida através de seu campo de velocidades (no referencial Euleriano). Se a evolução do fluido em coordenadas Lagrangianas é descrita por $\phi_{t}(x)$, seu campo de velocidades é determinado por:

$$
\vec{v}\left(\phi_{t}(x)\right)=\frac{\partial}{\partial t} \phi_{t}(x)
$$

No nosso contexto, $\phi_{t}(x)=Y(t) A(t) x, \operatorname{logo}, \vec{v}(y)=-\Omega y+Y \dot{A} A^{-1} Y^{T} y$. A vorticidade é dada por

$$
\nabla \times \vec{v} \equiv-2 \Omega+Y \dot{A} A^{-1} Y^{T}-Y A^{-1} \dot{A} Y^{T},
$$


onde novamente usamos a identificação entre vetores e matrizes antissimétricas. Desta forma, apesar de haver semelhanças entre as duas, elas não são idênticas (as expressões são iguais caso adotemos como campo de velocidades $\vec{v}(y)=G^{T} \dot{G} y$, com $G=Y A$ ).

Apesar de não persistir na presença desta dissipação por cisalhamento, esta vorticidade continua sendo conservada caso $\eta=0, \zeta \neq 0$, como indicado em (3.10).

Proposição 3.12. Suponha que $\mu>0$ (módulo de cisalhamento). Dado $E_{0} \geq 0$, o conjunto $E^{-1}\left(\left[0, E_{0}\right]\right) \subset T \operatorname{SSym}_{+}(3) \times \operatorname{skew}(3)$ é compacto.

Demonstração. Note que $E^{-1}\left(\left[0, E_{0}\right]\right)$ é fechado em $T \operatorname{SSym}_{+}(3) \times \operatorname{skew}(3)$ o qual é fechado em no conjunto $W=\mathrm{M}(3) \times \mathrm{M}(3) \times \operatorname{skew}(3)$. Mostraremos que não existe sequência $\left(A_{n}, \dot{A}_{n}, \Omega_{n}\right) \in$ $E^{-1}\left(\left[0, E_{0}\right]\right)$ que seja ilimitada em $W$. A definição de $E$, Proposição 3.4 , e a positividade de $T$ implicam

$$
\begin{gathered}
\frac{4 \pi \mu R^{3}}{3}\left(\operatorname{Tr}\left(A_{n}^{2}\right)-\frac{1}{3} \operatorname{Tr}\left(A_{n}\right)^{2}\right) \leq E \leq E_{0}, \quad \forall n, \\
\frac{\mathrm{I}_{\circ}}{4}\left(\left\|\dot{A}_{n}+\Omega_{n} A_{n}\right\|^{2}\right) \leq E \leq E_{0} . \quad \forall n .
\end{gathered}
$$

De (3.11) vemos que as normas dos vetores

$$
A_{n}-\frac{<A_{n}, \mathrm{Id}>}{\|\mathrm{Id}\|^{2}} \mathrm{Id}
$$

são limitadas. Assim, as projeções da sequência $A_{n}$ sobre qualquer vetor ortogonal a Id é limitada. Então, escrevendo $A_{n}=s_{n} \operatorname{Id}+\alpha_{n}$, onde $<\alpha_{n}$, Id $>=0$, vemos que a sequência $\left\|\alpha_{n}\right\|$ é limitada e que a sequência $\left\|A_{n}\right\|$ é ilimitada se, e somente se a sequência $\left|s_{n}\right|$ é ilimitada. Mas se $\left|s_{n}\right|$ é suficientemente grande, $s_{n}^{-1} \alpha_{n}$ é próxima de zero e $\operatorname{det}\left(A_{n}\right)=s_{n}^{3} \operatorname{det}\left(\operatorname{Id}+s_{n}^{-1} \alpha_{n}\right)>1$, o que é impossível pois $\operatorname{det}\left(A_{n}\right)=1$. Então $A_{n}$ é limitada. Denote $\left(A_{n}\right)_{i j}=a_{i j}^{n}$ e $\left(\dot{A}_{n}\right)_{i j}=b_{i j}^{n}$. A equação (3.12) mostra que $\dot{A}_{n}+\Omega_{n} A_{n}$ é limitada, assim como sua parte antissimétrica $\left(A_{n} \Omega_{n}+\Omega_{n} A_{n}\right) / 2$. Tomando $A_{n}$ diagonal novamente, vemos que $S\left(A_{n} \Omega_{n}+\Omega_{n} A_{n}\right)=\left(\left(a_{22}^{n}+a_{33}^{n}\right) \omega_{1}^{n},\left(a_{11}^{n}+a_{33}^{n}\right) \omega_{2}^{n},\left(a_{11}^{n}+\right.\right.$ $\left.a_{22}^{n}\right) \omega_{3}^{n}$ ) ( $S$ definido em 2.22). Desde que nenhum dos coeficientes $\left(a_{i i}^{n}+a_{j j}^{n}\right)$ pode se aproximar de zero, pois $a_{11}^{n} a_{22}^{n} a_{33}^{n}=1$, concluímos que a sequência $\Omega_{n}$ é limitada. Então $\dot{A}_{n}$ também deve ser limitada.

\subsubsection{Equilíbrios para o modelo não linear}

Para corpos sólidos, é comum adicionar a hipótese de incompressibilidade. Desta forma, para a análise dos equilíbrios, faremos a simplificação: $K=0$. Façamos também a reescala de tempo: $\tau=T t$ e $\tilde{\Omega}=\Omega / T$, onde

$$
T:=\sqrt{\frac{10 R^{3}}{3 M G}}
$$

Desta forma, nas novas coordenadas, a equação (3.9) não se altera, e (3.7) fica:

$$
\begin{gathered}
\ddot{A}+\frac{1}{2}[\dot{\Omega}, A]+[\Omega, \dot{A}]+\frac{1}{2}\left(\Omega^{2} A+A \Omega^{2}\right)+\frac{1}{\operatorname{Tr}\left(A^{-2}\right)}\left(2 \operatorname{Tr}\left(A^{-1} \dot{A} \Omega\right)-\operatorname{Tr}\left(A^{-1} \dot{A} A^{-1} \dot{A}\right)-\operatorname{Tr}\left(\Omega^{2}\right)\right) A^{-1}+ \\
+\frac{200 \pi R^{4}}{9 M^{2} G}\left(2 \mu A-\frac{2 \mu}{3} \operatorname{Tr}(A) \operatorname{Id}+\sqrt{\frac{3 M G}{10 R^{3}}}\left(2 \eta \dot{A}+\left(\zeta-\frac{2 \eta}{3}\right) \operatorname{Tr}(\dot{A}) \operatorname{Id}\right)+\right. \\
\left.-\frac{1}{\operatorname{Tr}\left(A^{-2}\right)}\left(6 \mu-\frac{2 \mu}{3} \operatorname{Tr}(A) \operatorname{Tr}\left(A^{-1}\right)+\sqrt{\frac{3 M G}{10 R^{3}}}\left(\zeta-\frac{2 \eta}{3}\right) \operatorname{Tr}(\dot{A}) \operatorname{Tr}\left(A^{-1}\right)\right)\right) A^{-1}+
\end{gathered}
$$




$$
+\int_{0}^{\infty} \frac{A\left(A^{2}+\lambda \mathrm{Id}\right)^{-1}}{\sqrt{\operatorname{det}\left(A^{2}+\lambda \mathrm{Id}\right)}} d \lambda-\frac{1}{\operatorname{Tr}\left(A^{-2}\right)}\left(\int_{0}^{\infty} \frac{\operatorname{Tr}\left(\left(A^{2}+\lambda \mathrm{Id}\right)^{-1}\right)}{\sqrt{\operatorname{det}\left(A^{2}+\lambda \mathrm{Id}\right)}} d \lambda\right) A^{-1}=0
$$

Sobre a questão dos equilíbrios, observamos o seguinte fato. Suponha que $\left(A_{1}, \Omega_{1}\right)$ pertença ao $\omega$-limite de alguma condição inicial $\left(A_{0}, \Omega_{0}\right)$ do sistema apresentado. Desde que um conjunto $\omega$-limite é invariante, pelo Princípio de LaSalle (Teorema 2.12), vemos que a solução $(A(t), \Omega(t)$ ) com $(A(0), \Omega(0))=\left(A_{1}, \Omega_{1}\right)$ é tal que mantem a função energia $E(t)=E(A(t), \Omega(t))$ constante. Pela Proposição 3.7, vemos que de fato $A(t)=A_{1}, \forall t$. Daqui em diante, este tipo de solução será denominada conservativa, sendo o único tipo possível de solução $\omega$-limite do sistema.

Suponha que o sistema anterior admita uma solução conservativa $\left(A_{0}, \Omega(t)\right) \in \operatorname{SSym}_{+}(3) \times$ skew(3). Note que para qualquer $Y_{0} \in \mathrm{SO}(3),\left(Y_{0} A_{0} Y_{0}^{T}, Y_{0} \Omega(t) Y_{0}^{T}\right)$ também é solução de (3.9) e (3.7), portanto podemos assumir que $A_{0}=\operatorname{diag}\left(a_{1}, a_{2}, a_{3}\right)$.

Desta forma, as equações resultam em:

$$
\begin{aligned}
& A_{0}^{2} \dot{\Omega}+\dot{\Omega} A_{0}^{2}+2 A_{0} \dot{\Omega} A_{0}+\left[\Omega^{2}, A_{0}^{2}\right]=0, \\
& \frac{1}{2}\left[\dot{\Omega}, A_{0}\right]+\frac{1}{2}\left(\Omega^{2} A_{0}+A_{0} \Omega^{2}\right)+D=0,
\end{aligned}
$$

onde $D$ é uma matriz diagonal.

Explicitamente, sendo

$$
\begin{aligned}
& \Omega(t)=\left(\begin{array}{ccc}
0 & -\omega_{3} & \omega_{2} \\
\omega_{3} & 0 & -\omega_{1} \\
-\omega_{2} & \omega_{1} & 0
\end{array}\right) \\
& \left\{\begin{array} { l } 
{ ( a _ { 1 } ^ { 2 } + a _ { 2 } ^ { 2 } ) \dot { \omega } _ { 3 } + 2 a _ { 1 } a _ { 2 } \dot { \omega } _ { 3 } - ( a _ { 2 } ^ { 2 } - a _ { 1 } ^ { 2 } ) \omega _ { 1 } \omega _ { 2 } = 0 } \\
{ ( a _ { 1 } ^ { 2 } + a _ { 3 } ^ { 2 } ) \dot { \omega } _ { 2 } + 2 a _ { 1 } a _ { 3 } \dot { \omega } _ { 2 } + ( a _ { 3 } ^ { 2 } - a _ { 1 } ^ { 2 } ) \omega _ { 1 } \omega _ { 3 } = 0 } \\
{ ( a _ { 2 } ^ { 2 } + a _ { 3 } ^ { 2 } ) \dot { \omega } _ { 1 } + 2 a _ { 2 } a _ { 3 } \dot { \omega } _ { 1 } - ( a _ { 3 } ^ { 2 } - a _ { 2 } ^ { 2 } ) \omega _ { 2 } \omega _ { 3 } = 0 }
\end{array} \Rightarrow \left\{\begin{array}{l}
\left(a_{1}+a_{2}\right) \dot{\omega}_{3}+\left(a_{1}-a_{2}\right) \omega_{1} \omega_{2}=0 \\
\left(a_{1}+a_{3}\right) \dot{\omega}_{2}+\left(a_{3}-a_{1}\right) \omega_{1} \omega_{3}=0 \\
\left(a_{2}+a_{3}\right) \dot{\omega}_{1}+\left(a_{2}-a_{3}\right) \omega_{2} \omega_{3}=0
\end{array}\right.\right. \\
& \left(\begin{array}{ccc}
-2 a_{1}\left(\omega_{2}^{2}+\omega_{3}^{2}\right) & \left(a_{2}-a_{1}\right) \dot{\omega}_{3}+\left(a_{1}+a_{2}\right) \omega_{1} \omega_{2} & \left(a_{1}-a_{3}\right) \dot{\omega}_{2}+\left(a_{1}+a_{3}\right) \omega_{1} \omega_{3} \\
\left(a_{2}-a_{1}\right) \dot{\omega}_{3}+\left(a_{1}+a_{2}\right) \omega_{1} \omega_{2} & -2 a_{2}\left(\omega_{1}^{2}+\omega_{3}^{2}\right) & \left(a_{3}-a_{2}\right) \dot{\omega}_{1}+\left(a_{2}+a_{3}\right) \omega_{2} \omega_{3} \\
\left(a_{1}-a_{3}\right) \dot{\omega}_{2}+\left(a_{1}+a_{3}\right) \omega_{1} \omega_{3} & \left(a_{3}-a_{2}\right) \dot{\omega}_{1}+\left(a_{2}+a_{3}\right) \omega_{2} \omega_{3} & -2 a_{3}\left(\omega_{1}^{2}+\omega_{2}^{2}\right)
\end{array}\right)+D=0 \\
& \Rightarrow\left\{\begin{array} { l } 
{ ( a _ { 2 } - a _ { 1 } ) \dot { \omega } _ { 3 } + ( a _ { 1 } + a _ { 2 } ) \omega _ { 1 } \omega _ { 2 } = 0 } \\
{ ( a _ { 1 } - a _ { 3 } ) \dot { \omega } _ { 2 } + ( a _ { 1 } + a _ { 3 } ) \omega _ { 1 } \omega _ { 3 } = 0 } \\
{ ( a _ { 3 } - a _ { 2 } ) \dot { \omega } _ { 1 } + ( a _ { 2 } + a _ { 3 } ) \omega _ { 2 } \omega _ { 3 } = 0 }
\end{array} \Rightarrow \left\{\begin{array}{l}
\left(\left(a_{1}+a_{2}\right)^{2}+\left(a_{2}-a_{1}\right)^{2}\right) \dot{\omega}_{3}=0 \\
\left(\left(a_{1}+a_{3}\right)^{2}+\left(a_{1}-a_{3}\right)^{2}\right) \dot{\omega}_{2}=0 \\
\left(\left(a_{2}+a_{3}\right)^{2}+\left(a_{3}-a_{2}\right)^{2}\right) \dot{\omega}_{1}=0
\end{array}\right.\right.
\end{aligned}
$$

Portanto, $\Omega(t)=\Omega_{0}$, constante.

Além disso, $\omega_{i} \omega_{j}=0, \forall i \neq j$. Portanto, podemos assumir que $\omega_{2}=\omega_{3}=0, \omega_{1}=\omega$.

Assim, de (3.13) vemos que $A, \Omega$ devem satisfazer:

$$
\Omega^{2} A^{2}+\alpha\left(A^{2}-\frac{1}{3} \operatorname{Tr}(A) A\right)+\int_{0}^{\infty} \frac{\left(\mathrm{Id}+\lambda A^{-2}\right)^{-1}}{\sqrt{\operatorname{det}\left(A^{2}+\lambda \mathrm{Id}\right)}} d \lambda=c \cdot \mathrm{Id} .
$$

Usando as entradas das matrizes, temos:

$$
\begin{gathered}
\alpha\left(a_{1}^{2}-\frac{1}{3} \operatorname{Tr}(A) a_{1}\right)+a_{1}^{2} \int_{0}^{\infty} \frac{1}{\sqrt{\operatorname{det}\left(A^{2}+\lambda \mathrm{Id}\right)}\left(a_{1}^{2}+\lambda\right)} d \lambda=c \\
-\omega^{2} a_{2}^{2}+\alpha\left(a_{2}^{2}-\frac{1}{3} \operatorname{Tr}(A) a_{2}\right)+a_{2}^{2} \int_{0}^{\infty} \frac{1}{\sqrt{\operatorname{det}\left(A^{2}+\lambda \mathrm{Id}\right)}\left(a_{2}^{2}+\lambda\right)} d \lambda=c
\end{gathered}
$$




$$
-\omega^{2} a_{3}^{2}+\alpha\left(a_{3}^{2}-\frac{1}{3} \operatorname{Tr}(A) a_{3}\right)+a_{3}^{2} \int_{0}^{\infty} \frac{1}{\sqrt{\operatorname{det}\left(A^{2}+\lambda \operatorname{Id}\right)}\left(a_{3}^{2}+\lambda\right)} d \lambda=c .
$$

Multiplicando a primeira equação por $a_{2}$, a segunda por $-a_{1}$ e somando-as, obtemos:

$$
\omega^{2} a_{2}^{2} a_{1}+\alpha a_{2} a_{1}\left(a_{2}-a_{1}\right)+a_{1} a_{2}\left(a_{2}-a_{1}\right) \int_{0}^{\infty} \frac{1}{\sqrt{\operatorname{det}\left(A^{2}+\lambda \mathrm{Id}\right)}} \frac{a_{1} a_{2}-\lambda}{\left(a_{1}^{2}+\lambda\right)\left(a_{2}^{2}+\lambda\right)} d \lambda=c\left(a_{2}-a_{1}\right) .
$$

Concluímos que se a velocidade angular do corpo for não nula, necessariamente $a_{2} \neq a_{1}$. E analogamente concluímos que $a_{3} \neq a_{1}$.

Em vista da existência de elipsoides triaxiais de equilíbrio (elipsoides de Jacobi) no problema de massas líquidas girantes [Cha87] é concebível que eles também figurem no presente contexto. Tratemos o caso dos elipsoides de revolução (ver discussão em torno da equação 4 na página 704 de [Lam32]).

Proposição 3.13. Assuma que $a_{2}=a_{3}$. Então para cada (norma do) momento angular, existe uma única configuração de equilíbrio, a menos de uma rotação.

Demonstração. Denote por $x:=a_{2}=a_{3}$. Como $\operatorname{det} A=1, a_{1}=1 / x^{2}$. Suponha ainda que exista uma tal configuração.

Note que o momento angular para uma rotação de equilíbrio é tal que:

$$
L^{2}=\frac{1}{4} \operatorname{Tr}\left(\left(A^{2} \Omega+\Omega A^{2}\right)^{T}\left(A^{2} \Omega+\Omega A^{2}\right)\right)=\frac{1}{4}\left(a_{2}^{2}+a_{3}^{2}\right)^{2} \operatorname{Tr}\left(\Omega^{T} \Omega\right)=2 x^{4} \omega^{2} .
$$

Assim, as equações (3.15) se reduzem a:

$$
\begin{gathered}
\frac{2}{3} \alpha\left(x^{-4}-x^{-1}\right)+x^{-4} \int_{0}^{\infty} \frac{1}{\left(x^{2}+\lambda\right)\left(x^{-4}+\lambda\right)^{\frac{3}{2}}} d \lambda=c \\
-\frac{L^{2}}{2} x^{-2}+\frac{1}{3} \alpha\left(x^{2}-x^{-1}\right)+x^{2} \int_{0}^{\infty} \frac{1}{\left(x^{2}+\lambda\right)^{2}\left(x^{-4}+\lambda\right)^{\frac{1}{2}}} d \lambda=c .
\end{gathered}
$$

Estas equações implicam em:

$$
\frac{\alpha}{3}\left(x^{6}+x^{3}-2\right)-\frac{L^{2}}{2} x^{2}=\left(1-x^{6}\right) \int_{0}^{\infty} \frac{\lambda}{\left(x^{2}+\lambda\right)^{2}\left(x^{-4}+\lambda\right)^{\frac{3}{2}}} d \lambda .
$$

Note que, para todo $x \in(0,1)$, o lado esquerdo da equação é negativo, e o lado direito positivo. Logo, se $x$ é solução desta equação, $x \geq 1$ (elipsoide oblato). Desta forma também concluímos que no caso $L=0$, a única solução é $x=1$ (esfera). A análise posterior se refere ao caso $L \neq 0$.

A integral ocorrendo acima admite uma expressão simples (consultar equação (A.1) do Apêndice A). Logo a configuração de equilíbrio com momento angular $L$ deve satisfazer:

$$
\frac{\alpha}{3}\left(x^{6}+x^{3}-2\right)-\frac{L^{2}}{2} x^{2}=\frac{x^{6}}{\left(x^{6}-1\right)^{\frac{3}{2}}}\left(\left(\tan ^{-1}\left(\left(x^{6}-1\right)^{-\frac{1}{2}}\right)-\frac{\pi}{2}\right)\left(x^{6}+2\right)+3 \sqrt{x^{6}-1}\right) .
$$

Note que

$$
\lim _{x \rightarrow \infty} \tan ^{-1}\left(\left(x^{6}-1\right)^{-\frac{1}{2}}\right)=0 .
$$

Logo, existe $N>1$ tal que $\forall x>N$ esta função é menor que $\pi / 4$. Logo, para todo $x>N$, o lado direito da equação (3.17) é menor que:

$$
\frac{x^{6}}{\left(x^{6}-1\right)^{\frac{3}{2}}}\left(-\frac{\pi}{4}\left(x^{6}+2\right)+3 \sqrt{x^{6}-1}\right) \stackrel{x \rightarrow \infty}{\longrightarrow}-\infty .
$$

Mas, claramente o lado direito da equação vai a $+\infty$ no mesmo limite. Portanto, para cada par $\alpha, L$ existe pelo menos uma solução $(x>1)$ da equação (desde que o lado direito é zero em $x=1$ ). 
Suponha agora que para algum par de parâmetros existam duas soluções distintas. Desta forma, existe $x>1$ tal que:

$$
\frac{\alpha}{3}\left(x^{6}+x^{3}-2\right)-\frac{L^{2}}{2} x^{2}-\frac{x^{6}}{\left(x^{6}-1\right)^{\frac{3}{2}}}\left(3 \sqrt{x^{6}-1}-\cot ^{-1}\left(\left(x^{6}-1\right)^{-\frac{1}{2}}\right)\left(x^{6}+2\right)\right) \geq 0,
$$

e a derivada desta expressão seja nula:

$$
\frac{\alpha}{3}\left(6 x^{5}+3 x^{2}\right)-L^{2} x-\frac{3 x^{5}}{\left(x^{6}-1\right)^{\frac{5}{2}}}\left[\cot ^{-1}\left(\left(x^{6}-1\right)^{-\frac{1}{2}}\right)\left(x^{12}-6 x^{6}-2\right)+\left(x^{6}+8\right) \sqrt{x^{6}-1}\right]=0 .
$$

Multiplique esta expressão por $-x / 2$ e some com a anterior para obter:

$-\frac{\alpha}{3}\left(4 x^{6}+x^{3}+4\right) \geq \frac{x^{6}}{2\left(x^{6}-1\right)^{3}}\left[\sqrt{x^{6}-1} \cot ^{-1}\left(\left(x^{6}-1\right)^{-\frac{1}{2}}\right)\left(x^{12}-20 x^{6}-8\right)+9\left(x^{12}+x^{6}-2\right)\right]$.

Note que

$$
\frac{d}{d y} \cot ^{-1}(y)=-\frac{1}{1+y^{2}} \geq-y^{-2}
$$

a qual integrada entre $y$ e $\infty$ fornece:

$$
\frac{\cot ^{-1}(y)}{y} \leq y^{-2}, \quad \forall y>0
$$

Concluímos daí que, nos valores de $x$ tais que $x^{12}-20 x^{6}-8 \leq 0$,

$$
-\frac{\alpha}{3}\left(4 x^{6}+x^{3}+4\right) \geq \frac{x^{6}}{2\left(x^{6}-1\right)^{3}}\left(x^{18}-12 x^{12}+21 x^{6}-10\right) \geq 0,
$$

onde a última desigualdade vale para todo $x \geq 1$, como se verifica facilmente pela derivada deste polinômio.

Caso o polinômio $x^{12}-20 x^{6}-8 \geq 0$, obtemos diretamente a mesma desigualdade (já que todos os termos envolvidos ficam positivos). Obtemos assim uma contradição, pois o lado esquerdo claramente é sempre negativo.

Só resta saber se este (único) valor de $x$ é solução do problema (3.14). Note que para as condições de equilíbrio, o lado direito da equação é dado por

$$
\frac{1}{\operatorname{Tr}\left(A^{-2}\right)}\left(\operatorname{Tr}\left(\Omega^{2}\right)+\alpha\left(3-\frac{1}{3} \operatorname{Tr}(A) \operatorname{Tr}\left(A^{-1}\right)\right)+\int_{0}^{\infty} \frac{\operatorname{Tr}\left(\left(A^{2}+\lambda \mathrm{Id}\right)^{-1}\right)}{\sqrt{\operatorname{det}\left(A^{2}+\lambda \mathrm{Id}\right)}} d \lambda\right) \text { Id. }
$$

Mas, acabamos de provar que para este valor de $x$, o lado esquerdo da equação (3.14) é um múltiplo da identidade (ver equação (3.17)). Ou seja,

$$
b A^{-2}=\Omega^{2}+\alpha\left(\operatorname{Id}-\frac{1}{3} \operatorname{Tr}(A) A^{-1}\right)+\int_{0}^{\infty} \frac{\left(A^{2}-\lambda \mathrm{Id}\right)}{\sqrt{\operatorname{det}\left(A^{2}+\lambda \mathrm{Id}\right)}} d \lambda .
$$

Tomando o traço desta expressão, vemos que $b=c$ da equação (3.14). Logo, $x$ solução da equação (3.17) corresponde à única solução de elipsoide de revolução (a menos de rotações) da equação (3.14).

Observação 3.14. Note que a Proposição 3.13 continua sendo verdadeira para $\alpha=0$. Neste caso, a equação para equilíbrios simétricos (3.16) é a mesma satisfeita pelos equilíbrios de MacLaurin, para o caso de massas fluidas (compare com as equações (5) e (6) do parágrafo 304 de [Lam32]). 
Uma pergunta pertinente é sobre a existência de equilíbrios alternativos aos elipsoides de revolução com a presença do potencial interno de deformação.

Nesta direção, uma resposta parcial pode ser fornecida. Retornando às equações (3.15), subtraindo a primeira delas das duas últimas, obtemos

$$
\begin{aligned}
& -\omega^{2} a_{2}^{2}+\left(a_{2}-a_{1}\right)\left(\frac{\alpha}{3}\left(2 a_{1}+2 a_{2}-a_{3}\right)+\left(a_{2}+a_{1}\right) \int_{0}^{\infty} \frac{\lambda}{\sqrt{\operatorname{det}\left(A^{2}+\lambda \mathrm{Id}\right)}\left(a_{1}^{2}+\lambda\right)\left(a_{2}^{2}+\lambda\right)} d \lambda\right)=0, \\
& -\omega^{2} a_{3}^{2}+\left(a_{3}-a_{1}\right)\left(\frac{\alpha}{3}\left(2 a_{1}+2 a_{3}-a_{2}\right)+\left(a_{3}+a_{1}\right) \int_{0}^{\infty} \frac{\lambda}{\sqrt{\operatorname{det}\left(A^{2}+\lambda \mathrm{Id}\right)}\left(a_{1}^{2}+\lambda\right)\left(a_{3}^{2}+\lambda\right)} d \lambda\right)=0 .
\end{aligned}
$$

Note que se considerarmos apenas a interação gravitacional, $\alpha=0$, uma solução de equilíbrio com momento angular não nulo (o que implica $\omega \neq 0$ ) deve satisfazer às desigualdades: $a_{1}<a_{2} \mathrm{e}$ $a_{1}<a_{3}$, pois as integrais acima são positivas.

Agora, nas equações (3.18), multiplique a primeira por $a_{3}^{2}$, a segunda por $-a_{2}^{2}$ e some-as, de forma a eliminar o $\omega^{2}$ :

$$
\begin{gathered}
\left(a_{2}-a_{3}\right)\left(\frac{\alpha}{3}\left(2 a_{1}^{2}\left(a_{2}+a_{3}\right)+a_{2}^{2}\left(a_{3}-a_{1}\right)+a_{3}^{2}\left(a_{2}-a_{1}\right)-1\right)+\right. \\
\left.+\left(a_{2}+a_{3}\right) \int_{0}^{\infty} \frac{\lambda\left(a_{1}^{2} \lambda+a_{1}^{2}\left(a_{2}^{2}+a_{3}^{2}\right)-a_{2}^{2} a_{3}^{2}\right)}{\left(\operatorname{det}\left(A^{2}+\lambda\right)\right)^{\frac{3}{2}}} d \lambda\right)=0 .
\end{gathered}
$$

Supondo agora que $a_{2} \neq a_{3}$, concluímos:

$$
\frac{\alpha}{3}\left(2 a_{1}^{2}\left(a_{2}+a_{3}\right)+a_{2}^{2}\left(a_{3}-a_{1}\right)+a_{3}^{2}\left(a_{2}-a_{1}\right)-1\right)+\left(a_{2}+a_{3}\right) \int_{0}^{\infty} \frac{\lambda\left(a_{1}^{2} \lambda+a_{1}^{2}\left(a_{2}^{2}+a_{3}^{2}\right)-a_{2}^{2} a_{3}^{2}\right)}{\left(\operatorname{det}\left(A^{2}+\lambda\right)\right)^{\frac{3}{2}}} d \lambda=0 .
$$

Novamente fazendo $\alpha=0$, obtemos a condição de equilíbrio relacionada ao problema de fluidos girantes, a equação que determina os elipsoides de Jacobi (triaxiais), ver equação (4) do parágrafo 375 de [Lam32]. Neste caso, é bem conhecido (desde Jacobi) que existem estes tipos de equilíbrio, mas que somente podem ocorrer distantes do formato esférico, acima de um certo valor de momento angular, ou em outras palavras, a família (a um parâmetro) de equilíbrios no formato de elipsoide triaxial bifurca da família de elipsoides de revolução (de MacLaurin), longe do formato $a_{1}=a_{2}=$ $a_{3}=1$. Por ilustração, segue a Tabela 3.1 extraída de [Dar86].

\begin{tabular}{|c|c|c|c|c|}
\hline \multicolumn{3}{|c|}{ Eixo } & Velocidade angular & Momento angular \\
\hline$a_{1}$ & $a_{2}$ & $a_{3}$ & $\frac{\omega^{2}}{4 \pi \rho}$ & $L$ \\
\hline 1,1972 & 1,1972 & 0,6977 & 0,09356 & 0,30375 \\
\hline 1,216 & 1,179 & 0,698 & 0,094 & 0,304 \\
\hline 1,279 & 1,123 & 0,696 & 0,093 & 0,306 \\
\hline 1,3831 & 1,0454 & 0,6916 & 0,09060 & 0,3134 \\
\hline 1,6007 & 0,9235 & 0,6765 & 0,08295 & 0,3407 \\
\hline 1,899 & 0,8111 & 0,6494 & 0,07047 & 0,3920 \\
\hline 2,346 & 0,7019 & 0,6072 & 0,0536 & 0,4809 \\
\hline 3,136 & 0,5858 & 0,5445 & 0,0334 & 0,644 \\
\hline 5,04 & 0,45 & 0,44 & 0,013 & 1,016 \\
\hline$\infty$ & 0 & 0 & 0 & $\infty$ \\
\hline
\end{tabular}

Tabela 3.1: Família a um parâmetro dos elipsoides de Jacobi

Note que as condições (3.19) e $\operatorname{det}(A)=1$ fixam dois parâmetros em termos do terceiro, o que reduz o problema à série ilustrada acima, a qual termina em um elipsoide oblato para o momento 
angular mais baixo. Observe também que ao longo desta série, para momentos angulares muito altos, o formato do corpo se aproxima de um elipsoide prolato muito alongado, semelhante a um tubo. Não foi possível tirar conclusões completas sobre a ocorrência de elipsoides triaxiais quando $\alpha>0$. As ideias clássicas não se adaptam para este caso.

Quando $\alpha>0$, também não podem ocorrer elipsoides triaxiais (de volume $4 \pi / 3$, fixando $R=1$ sem perda de generalidade) arbitrariamente próximos da bola de raio 1 . De fato, suponha que exista uma sequência de triplas $\left(a_{1 i}, a_{2 i}, a_{3 i}\right), a_{j i}>0$, que satisfaçam (3.19), com $a_{2 i} \neq a_{3 i}$, para todo $i \in \mathbb{N}$, e que ainda convirja para a tripla $(1,1,1)$ quando $i \rightarrow+\infty$. Note que as funções das variáveis $\left(a_{1}, a_{2}, a_{3}\right)$ na expressão (3.19) são contínuas (a integral imprópria é sempre convergente). Portanto, no limite $i \rightarrow+\infty$, a equação se reduz a uma contradição:

$$
0=\alpha+2 \int_{0}^{\infty} \frac{\lambda(\lambda+1)}{(1+\lambda)^{\frac{9}{2}}} d \lambda=\alpha+\frac{4}{15}
$$

Observe que o raciocínio acima também nos assegura que existe uma vizinhança do ponto $\left(a_{1}, a_{2}, a_{3}\right)=(1,1,1)$ na qual todos os equilíbrios são tais que $a_{2}=a_{3}$, logo os termos $2 a_{1}+2 a_{2}-a_{3}$ e $2 a_{1}+2 a_{3}-a_{2}$ que ocorrem em (3.18) são positivos. E assim, pela mesma equação, concluímos que $a_{2}=a_{3}>a_{1}$, obtendo um elipsoide de revolução oblato.

Podemos assim concluir esta seção com o seguinte resultado, consequência do Princípio de LaSalle, Teorema 2.12.

Teorema 3.15. Para $\mu>0$, toda solução das equaçôes (3.7) e (3.8) com baixo momento angular $L$ é atraída para o único equilíbrio relativo com este dado valor de momento angular. O formato assintótico é um elipsoide de revolução oblato.

Observamos que um teorema similar a este vale no caso $\mu=0$. Neste caso, algumas limitações adicionais da energia devem ser impostas. A força gravitacional não é forte o suficiente para restringir o crescimento de condições iniciais muito energéticas.

\subsection{Corpo pseudo-rígido com pequenas deformações}

Note que $\operatorname{SSym}_{+}(3)$ não é um grupo de Lie, pois não é fechado por multiplicação de matrizes, mas também possui uma função exponencial associada (para mais detalhes sobre este conjunto, ver [FM03]). Como foi discutido na seção anterior, de (3.2) sabemos que o espaço tangente à identidade é

$$
\operatorname{ssym}(3):=T_{\text {Id }} \operatorname{SSym}_{+}(3) \equiv\{B \in \operatorname{Sym}(3): \operatorname{Tr}(B)=0\} .
$$

Considere a exponencial de matrizes usual. Temos que, para todo $B \in \operatorname{ssym}(3), \exp (B)^{T}=$ $\exp (B)$ e $\operatorname{det}(\exp (B))=e^{\operatorname{Tr}(B)}=1$. Ou seja, $\exp (\operatorname{ssym}(3)) \subset \operatorname{SSym}_{+}(3)$.

Proposição 3.16. A aplicação $\exp : \operatorname{ssym}(3) \longrightarrow \operatorname{SSym}_{+}(3)$ é um difeomorfismo (global).

Demonstração. É um fato bem conhecido que exp é um difeomorfismo local em 0. Basta lembrar que

$$
d \exp _{0}(B)=\left.\frac{d}{d t}\right|_{t=0} \exp (t B)=B, \forall B
$$

ou seja, existe uma bola de raio $\varepsilon$ em torno de $0, V_{\varepsilon}$ (na métrica Euclidiana), sobre a qual exp é difeomorfismo.

Suponha que existam $B, C \in \operatorname{ssym}(3)$ tais que $\exp (B)=\exp (C)$. Tomando $n \in \mathbb{N}$ tal que $n^{-1} B, n^{-1} C \in V_{\varepsilon}$, concluímos que $\exp \left(n^{-1} B\right)^{n}=\exp (B)=\exp (C)=\exp \left(n^{-1} C\right)^{n}$. Como estas matrizes são simétricas, positivas definidas, obtemos que $\exp \left(n^{-1} B\right)=\exp \left(n^{-1} C\right)$. Logo $B=C$, isto é, exp é injetora. 
Dada $A \in \operatorname{SSym}_{+}(3)$, tome $Y \in O(3)$ tal que $Y A Y^{T}=\operatorname{diag}\left(a_{1}, a_{2}, a_{3}\right)$, com $a_{i}>0, i=$ $1,2,3$ e $a_{1} a_{2} a_{3}=1$. Assim, tomando $B=Y^{T} \operatorname{diag}\left(\ln \left(a_{1}\right), \ln \left(a_{2}\right), \ln \left(a_{3}\right)\right) Y$, temos que $\exp (B)=$ $Y^{T} \operatorname{diag}\left(a_{1}, a_{2}, a_{3}\right) Y=A$. Logo, é sobrejetora.

Dados $B \in V_{\varepsilon}$ e $H \in \operatorname{ssym}(3), H \neq 0$, sabemos que $d \exp _{B}(H) \neq 0$. Sendo assim, suponha que

$$
\left.\frac{d}{d t}\right|_{t=0} \exp (2 B+2 t H)=d \exp _{B}(H) \exp (B)+\exp (B) d \exp _{B}(H)=0 .
$$

Assumimos, sem perda de generalidade, que $\exp (B)$ seja diagonal. Lembrando que $\exp (B)$ é positiva e que $d \exp _{B}(H)$ também é simétrica, verificamos que a igualdade acima não pode ser satisfeita. Ou seja, $d \exp _{2 B}(H) \neq 0$, e por simples indução, $d \exp _{2^{n} B}(H) \neq 0, \forall n \in \mathbb{N}$.

Desta forma, dado $B_{0} \in \operatorname{ssym}(3)$, tome $n \in \mathbb{N}$ tal que $2^{-n} B_{0} \in V_{\varepsilon}$. Logo, para todo $H \in \operatorname{ssym}(3)$ não nulo, $d \exp _{B_{0}}(H)=d \exp _{2^{n}\left(2^{-n} B_{0}\right)}(H) \neq 0$.

Portanto, exp é difeomorfismo local em todo ponto e, portanto, difeomorfismo global.

O próximo objetivo é estudar uma equação de movimento aproximada, para um regime de baixas energias relativamente a níveis de momento angular fixos, de forma que o corpo sofra apenas pequenas deformações. Para tanto, será necessário usar o sistema de coordenadas apresentado na Proposição 3.16. Com o objetivo de entender a magnitude relativa das variáveis $A \in \mathrm{SSym}_{+}(3)$ e $\Omega \in$ skew(3) neste regime, analisemos a equação de equilíbrio (3.17) do sistema original, lembrando que para pequenas deformações o único formato de equilíbrio admissível é de um elipsoide de revolução oblato.

A Proposição 3.13 garantiu a existência de um único formato de equilíbrio para cada valor de momento angular. Analisando a equação (3.16), observamos que tal formato para momento angular nulo é o esférico $(x=1)$ e oblato para pequenos valores do parâmetro $L, x=\exp (\varepsilon b), \operatorname{com} \varepsilon \approx 0$. Note que, desta forma, a equação (3.16) fica

$$
\omega^{2}(\varepsilon)=\frac{\alpha}{3}\left(1+e^{-3 \varepsilon}-2 e^{-6 \varepsilon}\right)+\left(1-e^{-6 \varepsilon}\right) \int_{0}^{\infty} \frac{\lambda}{\left(e^{2 \varepsilon}+\lambda\right)^{2}\left(e^{-4 \varepsilon}+\lambda\right)^{\frac{3}{2}}} d \lambda .
$$

Neste caso particular, pudemos inverter trivialmente a equação, obtendo uma função suave $\omega^{2}(\varepsilon)$.

Derivando com respeito a $\varepsilon$, calculando em 0 e observando que $\omega(0)=0$, obtemos:

$$
\left.\frac{d}{d \varepsilon}\right|_{\varepsilon=0} \omega^{2}(\varepsilon)=3 \alpha+6 \int_{0}^{\infty} \frac{\lambda}{(1+\lambda)^{2}(1+\lambda)^{\frac{3}{2}}} d \lambda=3 \alpha+\frac{24}{15}>0 .
$$

Portanto, $\omega^{2}(\varepsilon)=\mathcal{O}(\varepsilon)$, ou seja, $\omega(\varepsilon)=\mathcal{O}(\sqrt{\varepsilon})$. Com base neste cálculo, propomos novas coordenadas para esse problema: $A=\exp (\varepsilon B), \Omega=\varepsilon^{-\frac{1}{2}} \tilde{\Omega}$.

Nestas coordenadas, tomando por simplicidade de notação $\tilde{\Omega}=\Omega$, a energia cinética até ordem $\varepsilon^{2}$ fica

$$
T=\frac{\mathrm{I}_{\circ}}{4}\left(\varepsilon^{2} \operatorname{Tr}\left(\dot{B}^{2}\right)-\varepsilon \operatorname{Tr}\left(\Omega^{2}\right)-2 \varepsilon^{2} \operatorname{Tr}\left(\Omega^{2} B\right)\right)+\mathcal{O}\left(\varepsilon^{\frac{5}{2}}\right) .
$$

Observação 3.17. Aproximando a energia cinética com o critério acima, o próximo termo seria $\varepsilon^{5 / 2} \operatorname{Tr}(B \dot{B} \Omega)$, a partir do qual vamos desprezar. A aproximação até ordem $\varepsilon$ não fornece informação sobre a dinâmica.

Usando novamente que $\operatorname{Tr}(B)=0$, calculamos o polinômio de Taylor de (3.5) até ordem $\varepsilon^{2}$

$$
V(B)=\varepsilon^{2} \frac{4 \pi \mu R^{3}}{3} \operatorname{Tr}\left(B^{2}\right)+\mathcal{O}\left(\varepsilon^{3}\right),
$$


e para a função de Rayleigh,

$$
\mathcal{D}(\dot{B})=\varepsilon^{2} \frac{4 \pi \eta R^{3}}{3} \operatorname{Tr}\left(\dot{B}^{2}\right)+\mathcal{O}\left(\varepsilon^{3}\right)
$$

Usando novamente a métrica Euclidiana em $\mathrm{M}(3)$, temos que a projeção ortogonal $P: \mathrm{M}(3) \longrightarrow$ $\operatorname{ssym}(3)$ é dada pela Proposição 3.8:

$$
P(H)=\frac{1}{2}\left(H+H^{T}\right)-\frac{1}{3} \operatorname{Tr}(H) \operatorname{Id} .
$$

Assim, desprezando os termos de ordem $\mathcal{O}\left(\varepsilon^{5 / 2}\right)$ e usando o Teorema 2.2, as equações de movimento para $B$ ficam

$$
P\left(\frac{d}{d t}\left(\frac{\partial \mathcal{L}}{\partial \dot{B}}\right)-\frac{\partial \mathcal{L}}{\partial B}+\frac{\partial \mathcal{D}}{\partial \dot{B}}\right)=0
$$

$\operatorname{logo}$,

$$
\ddot{B}+\frac{16 \pi \eta R^{3}}{3 \mathrm{I}_{\circ}} \dot{B}+\frac{16 \pi \mu R^{3}}{3 \mathrm{I}_{\circ}} B=-\Omega^{2}+\frac{1}{3} \operatorname{Tr}\left(\Omega^{2}\right) \mathrm{Id} .
$$

Podemos aqui também adicionar a energia gravitacional do corpo sobre ele próprio. Usando o resultado (B.7) do Apêndice B,

$$
V_{1}(B)=\frac{1}{8} G\left(7 h_{0}-9 h_{1}\right) \varepsilon^{2} \operatorname{Tr}\left(B^{2}\right)+\mathcal{O}\left(\varepsilon^{3}\right),
$$

onde os coeficientes $h_{0}$ e $h_{1}$ são obtidos de (B.8) e (B.9), respectivamente. No caso homogêneo, obtemos de (B.6)

$$
V_{1}(B)=\varepsilon^{2} \frac{G M^{2}}{R} \frac{2}{25} \operatorname{Tr}\left(B^{2}\right)+\mathcal{O}\left(\varepsilon^{3}\right) .
$$

Assim, a equação para a deformação fica:

$$
\ddot{B}+\nu \dot{B}+\tilde{\mu} B=-\Omega^{2}+\frac{1}{3} \operatorname{Tr}\left(\Omega^{2}\right) \mathrm{Id},
$$

onde $\nu$ e $\tilde{\mu}$ são as constantes de dissipação e elástica efetivas do corpo. No caso homogêneo, temos

$$
\nu:=\frac{40 \pi \eta R}{3 M}, \quad \tilde{\mu}:=\frac{4}{5} \frac{G M}{R^{3}}\left(1+\frac{25}{2} \frac{\mu}{g \rho R}\right)
$$

onde $g$ é a aceleração gravitacional na superfície do corpo.

Note que, fixos $\rho$ e $\mu$, quanto maior for o raio do corpo, menos significante é a sua elasticidade.

Calculando a equação para a parte rotacional de maneira análoga, usando o Lema 2.6, obtemos:

$$
\begin{gathered}
\dot{\Omega}+\varepsilon\left(\dot{\Omega} B+B \dot{\Omega}+\Omega \dot{B}+\dot{B} \Omega+\left[\Omega^{2}, B\right]\right)=0 \\
\Phi_{\varepsilon B}(\dot{\Omega})=\varepsilon\left(-\Omega \dot{B}-\dot{B} \Omega+\left[B, \Omega^{2}\right]\right)
\end{gathered}
$$

onde definimos o operador linear,

$$
\Phi_{\varepsilon B}(H):=H+\varepsilon B H+\varepsilon H B, \quad \forall H \in \operatorname{skew}(3) .
$$

Lema 3.18. Para todo $B \in \operatorname{ssym}(3),\|B\|<1 / 2 \varepsilon$, o operador $\Phi_{\varepsilon B}: \operatorname{skew}(3) \longrightarrow \operatorname{skew}(3)$ é simétrico, positivo definido.

Demonstração. Para todos $H_{1}, H_{2} \in \operatorname{skew}(3)$,

$$
\left\langle\Phi_{\varepsilon B}\left(H_{1}\right), H_{2}\right\rangle=-\operatorname{Tr}\left(H_{1} H_{2}\right)-\varepsilon \operatorname{Tr}\left(H_{1} B H_{2}\right)-\varepsilon \operatorname{Tr}\left(B H_{1} H_{2}\right)=\left\langle H_{1}, \Phi_{\varepsilon B}\left(H_{2}\right)\right\rangle .
$$


Mostremos que é inversível. Seja $H$ tal que $H+\varepsilon B H+\varepsilon H B=0 \Rightarrow(\operatorname{Id}+2 \varepsilon B) H+H(\operatorname{Id}+2 \varepsilon B)=$ 0 . Tome $v \in \mathbb{R}^{3}$, autovetor unitário de $(\mathrm{Id}+2 \varepsilon B)$. Logo, $\exists \lambda \in \mathbb{R}$ :

$$
v+2 \varepsilon B v=\lambda v \Rightarrow B v=\frac{\lambda-1}{2 \varepsilon} v \Rightarrow \frac{|\lambda-1|}{2 \varepsilon}<\frac{1}{2 \varepsilon} .
$$

Portanto, $\lambda>0$, i.e, $A=(\operatorname{Id}+2 \varepsilon B)$ é simétrica, positiva definida. Tomando coordenadas nas quais $A=\operatorname{diag}\left(a_{1}, a_{2}, a_{3}\right)$, e

$$
H=\left(\begin{array}{ccc}
0 & -h_{3} & h_{2} \\
h_{3} & 0 & -h_{1} \\
-h_{2} & h_{1} & 0
\end{array}\right)
$$

vemos que esta equação é equivalente a:

$$
\left(\begin{array}{ccc}
0 & -\left(a_{1}+a_{2}\right) h_{3} & \left(a_{1}+a_{3}\right) h_{2} \\
\left(a_{1}+a_{2}\right) h_{3} & 0 & -\left(a_{2}+a_{3}\right) h_{1} \\
-\left(a_{1}+a_{3}\right) h_{2} & \left(a_{2}+a_{3}\right) h_{1} & 0
\end{array}\right)=0 .
$$

Logo, $H=0$.

Sendo $\lambda$ e $H$ autovalor e autovetor de $\Phi_{\varepsilon B}$, temos

$$
(\lambda-1) H=\varepsilon(B H+H B) \Rightarrow|\lambda-1| \leq 2 \varepsilon\|B\| \leq 1 .
$$

Portanto, como $\lambda \neq 0, \lambda \in(0,2)$.

Aqui também temos que (quando a solução estiver bem definida) $\dot{E}=-2 \mathcal{D} \leq 0$, e $E(t)$ é constante se, e só se, $\dot{B} \equiv 0$. De forma análoga ao cálculo na Proposição 3.10 , obtemos a quantidade conservada (momento angular):

$$
L(B, \dot{B}, \Omega)=Y(\Omega+\varepsilon \Omega B+\varepsilon B \Omega) Y^{T}=Y \Phi_{\varepsilon B}(\Omega) Y^{T} .
$$

Note que, neste caso, se $(B(t), \Omega(t))$ for conservativa, $B(t)=B_{0}$, constante. Logo,

$$
\begin{gathered}
B=-\tilde{\mu}^{-1}\left(\Omega^{2}-\frac{1}{3} \operatorname{Tr}\left(\Omega^{2}\right) \mathrm{Id}\right) \Rightarrow\left[B, \Omega^{2}\right]=0 \\
\Rightarrow(\mathrm{Id}+2 \varepsilon B) \Omega+\Omega(\operatorname{Id}+2 \varepsilon B)=\text { constante } \Rightarrow \Omega(t)=\text { constante } .
\end{gathered}
$$

Desta forma, a configuração de equilíbrio consiste no elipsoide definido pela exponencial desta matriz. Notamos que no equilíbrio o momento angular (3.28) fica na forma

$$
L=\left(1+\frac{4}{3} \frac{\omega^{2}}{\tilde{\mu}} \varepsilon\right) \Omega_{0} \Rightarrow\|L\|=\left(1+\frac{4}{3} \frac{\omega^{2}}{\tilde{\mu}} \varepsilon\right) \omega
$$

onde $\Omega_{0}$ é a velocidade angular de equilíbrio.

Note que tomando a rotação estacionária em torno do eixo $x$,

$$
\Omega_{0}=\omega\left(\begin{array}{ccc}
0 & 0 & 0 \\
0 & 0 & -1 \\
0 & 1 & 0
\end{array}\right)
$$

a matriz de deformação no equilíbrio fica

$$
A_{0}=\exp \left(\frac{\omega^{2}}{\tilde{\mu}}\left(\begin{array}{ccc}
-2 / 3 & 0 & 0 \\
0 & 1 / 3 & 0 \\
0 & 0 & 1 / 3
\end{array}\right)\right)=\left(\begin{array}{ccc}
e^{-\frac{2 \omega^{2}}{3 \tilde{\mu}}} & 0 & 0 \\
0 & e^{\frac{\omega^{2}}{3 \tilde{\mu}}} & 0 \\
0 & 0 & e^{\frac{\omega^{2}}{3 \tilde{\mu}}}
\end{array}\right)
$$


Esta estimativa é a mesma obtida na equação (6.37) de [Fit12]. Mais precisamente, a quantidade apresentada é a eliticidade do elipsoide (oblato), definida por

$$
\epsilon=\frac{a-b}{R}
$$

onde $a$ e $b$ são os comprimentos do maior e do menor semi-eixo, respectivamente. Neste caso, obtivemos:

$$
\epsilon=e^{\frac{\omega^{2}}{3 \tilde{\mu}}}-e^{-\frac{2 \omega^{2}}{3 \tilde{\mu}}} \approx \frac{\omega^{2}}{\tilde{\mu}}=\frac{1}{2} \frac{\omega^{2} R^{3}}{G M} h_{2}, \quad h_{2}:=\frac{5}{2} \frac{1}{\left(1+\frac{25}{2} \frac{\mu}{\rho g R}\right)},
$$

onde $h_{2}$ faz o papel de um número de Love. O valor encontrado na literatura para este número, cujo cálculo pode ser encontrado em [Lov44] ou [TT09], é

$$
h_{2}=\frac{5}{2} \frac{1}{\left(1+\frac{19}{2} \frac{\mu}{\rho g R}\right)} .
$$

No Apêndice E encontram-se mais detalhes sobre este problema.

Caso seja desprezada a elasticidade do corpo $(\mu=0)$, a eliticidade assume o mesmo valor da equação (5.96) de [TS02] (ver também (1.7)):

$$
\epsilon \approx \frac{5}{4} \frac{\omega^{2} R^{3}}{G M}
$$

Proposição 3.19. Seja $\beta=\mathrm{I}_{\circ} \tilde{\mu} / 20$. A componente conexa de

$$
\mathcal{V}:=\left\{(B, U, \Omega) \in E^{-1}([0, \beta)):\|B\|<1 / 2 \varepsilon\right\}
$$

que contém a origem é tal que vale a existência e unicidade de soluções. Esta componente ainda é invariante e limitada.

Demonstração. Lembre que a energia é dada por:

$$
E(B, \dot{B}, \Omega)=\frac{\mathrm{I}_{\circ}}{4}\left(\varepsilon^{2} \operatorname{Tr}\left(\dot{B}^{2}\right)-\varepsilon \operatorname{Tr}\left((\mathrm{Id}+2 \varepsilon B) \Omega^{2}\right)+\varepsilon^{2} \tilde{\mu} \operatorname{Tr}\left(B^{2}\right)\right) .
$$

Note que nesta região, $A=\operatorname{Id}+2 \varepsilon B>0$, logo $-\operatorname{Tr}\left((\operatorname{Id}+2 \varepsilon B) \Omega^{2}\right)=\operatorname{Tr}\left((\sqrt{A} \Omega)^{T}(\sqrt{A} \Omega)\right) \geq 0$.

Tomando uma condição inicial neste conjunto, o Lema 3.18 nos garante que existe uma única solução local associada. Ao longo desta solução, sabemos que $E(t)<\beta$. Suponha que exista uma sequência de tempos $\left\{t_{n}\right\}$ tais que $\left\|B\left(t_{n}\right)\right\|<1 / 2 \varepsilon, \forall n$, e $\lim _{n \rightarrow+\infty}\left\|B\left(t_{n}\right)\right\|=1 / 2 \varepsilon$. Mas, neste caso,

$$
E(0) \geq E\left(t_{n}\right) \geq \varepsilon^{2} \frac{\mathrm{I}_{\circ} \tilde{\mu}}{4}\left\|B\left(t_{n}\right)\right\|^{2} \Rightarrow E(0) \geq \frac{\mathrm{I}_{\circ} \tilde{\mu}}{16} .
$$

Obtemos, assim, uma contradição. Assim, de fato, o conjunto é invariante.

Note ainda que, $\forall(B, \dot{B}, \Omega) \in \mathcal{V},\|B\|<1 / 2 \varepsilon,\|\dot{B}\|^{2}<4 \beta / \mathrm{I}_{\circ} \varepsilon^{2},\|\sqrt{A} \Omega\|^{2}<4 \beta / \mathrm{I}_{\circ} \varepsilon$.

Proposição 3.20. Seja $L^{2}(\Omega, B, \dot{B}):=\left\|\Phi_{\varepsilon B}(\Omega)\right\|^{2}$, o quadrado da norma do momento angular. Para todo $c>0,\left(L^{2}\right)^{-1}(c) \cap \mathcal{V}$ é subvariedade (de codimensão 1 ).

Demonstração. Suponha que para todo $\left(H_{1}, H_{2}, H_{3}\right) \in \operatorname{skew}(3) \times \operatorname{SSym}_{0}(3)^{2}$, $D L^{2}(\Omega, B, \dot{B})\left(H_{1}, H_{2}, H_{3}\right)=-\left(2 \operatorname{Tr}\left(\Omega H_{1}\right)+8 \varepsilon \operatorname{Tr}\left(B \Omega H_{1}\right)+4 \varepsilon^{2} \operatorname{Tr}\left(B^{2} \Omega H_{1}\right)+4 \varepsilon^{2} \operatorname{Tr}\left(B \Omega B H_{1}\right)\right.$, $\left.4 \varepsilon \operatorname{Tr}\left(\Omega^{2} H_{2}\right)+4 \varepsilon^{2} \operatorname{Tr}\left(\Omega^{2} B H_{2}\right)+4 \varepsilon^{2} \operatorname{Tr}\left(\Omega B \Omega H_{2}\right), 0\right)=(0,0,0)$. 
Assim, o segundo termo da equação implica que:

$$
(\Omega+\varepsilon B \Omega+\varepsilon \Omega B) \Omega=0 \Rightarrow\left\langle\Phi_{\varepsilon B}(\Omega), \Omega\right\rangle=0 .
$$

Usando o Lema 3.18, garantimos que a equação acima implica $\Omega=0$. Logo, $(\Omega, B, \dot{B})$ não pertence ao conjunto em questão, e $c$ é valor regular.

Note que no caso $c=0,\left(L^{2}\right)^{-1}(c) \cap \mathcal{V}=\{0\} \times \operatorname{ssym}(3)^{2}$ é subvariedade de codimensão 3 .

Portanto, como no caso anterior, podemos usar o Teorema 2.12 para concluir que a solução associada a qualquer condição em $\mathcal{V}$, definido em (3.34) se aproxima assintoticamente de um movimento de rotação estacionária, sendo que o corpo tem um formato de elipsoide oblato com eixo de revolução alinhado com o eixo de rotação.

Esta aproximação da deformação na identidade simplifica muito a análise do sistema. Isto ficará mais evidente no tratamento do problema de dois corpos.

\subsection{Estabilidade linear}

Recordamos de [Car81] alguns resultados básicos de variedades centrais em Equações Diferenciais, as quais aplicamos a este modelo. No caso geral, tome $x \in \mathbb{R}^{n}, y \in \mathbb{R}^{m}$, e sejam $A_{1} \in \mathrm{M}(\mathrm{n})$, $A_{2} \in \mathrm{M}(\mathrm{m})$ matrizes quadradas, $f: \mathbb{R}^{n+m} \longrightarrow \mathbb{R}^{n}$ e $g: \mathbb{R}^{n+m} \longrightarrow \mathbb{R}^{m}$ funções suaves tais que $f(0,0)=0, D f(0,0)=0, g(0,0)=0$ e $D g(0,0)=0$. Considere então o sistema

$$
\left\{\begin{array}{l}
\dot{x}=A_{1} x+f(x, y) \\
\dot{y}=A_{2} y+g(x, y)
\end{array},\right.
$$

supondo que todos os autovalores de $A_{1}$ tenham parte real nula e que todos os autovalores de $A_{2}$ tenham parte real negativa.

Seja $h: \mathbb{R}^{n} \longrightarrow \mathbb{R}^{m}$, com $h(0)=0$ e $D h(0)=0$, uma função suave. Se seu gráfico $y=h(x)$ é uma variedade invariante pelo fluxo de (3.35), ele será chamado de uma variedade central para (3.35). Neste caso o fluxo nessa variedade é dado por $(u(t), h(u(t)))$, onde $u(t) \in \mathbb{R}^{n}$ é solução de

$$
\dot{u}=A_{1} u+f(u, h(u)) .
$$

Assim, temos o seguinte teorema, provado em [Car81].

Teorema 3.21. Suponha que a solução nula de (3.36) seja estável. Então

(i) A solução nula de (3.35) é estável.

(ii) Seja $(x(t), y(t))$ uma solução de (3.35), com $(x(0), y(0))$ suficientemente pequeno, e $\sigma:=$ $\min \left\{|\operatorname{Re}(\lambda)|: \lambda \in \operatorname{Espectro}\left(A_{2}\right)\right\}$. Então, existem constantes $C_{1}, C_{2}>0$ e uma solução $u(t)$ de (3.36) tais que

$$
\|x(t)-u(t)\| \leq C_{1} e^{-\sigma t}, \quad\|y(t)-h(u(t))\| \leq C_{2} e^{-\sigma t} .
$$

Em nosso caso, as equações (3.24) e (3.26) podem ser escritas como

$$
\begin{gathered}
\dot{\Omega}=\varepsilon \Phi_{\varepsilon B}^{-1}\left(\left[B, \Omega^{2}\right]-U \Omega-\Omega U\right), \\
\left(\begin{array}{c}
\dot{B} \\
\dot{U}
\end{array}\right)=A_{2}\left(\begin{array}{c}
B \\
U
\end{array}\right)+\left(\begin{array}{c}
0 \\
-\Omega^{2}+1 / 3 \operatorname{Tr}\left(\Omega^{2}\right) \mathrm{Id}
\end{array}\right),
\end{gathered}
$$

onde $A_{2}(B, U):=(U,-\nu U-\tilde{\mu} B)$. Note que este sistema tem a forma (3.35), com $x=\Omega, y=(B, U)$ e $A_{1}(\Omega)=0$. O único autovalor de $A_{1}$ é 0 . Se $A_{2}(B, U)=\lambda(B, U)$, então $\lambda^{2}+\nu \lambda+\tilde{\mu}=0, \operatorname{logo}$

$$
\lambda=-\frac{\nu}{2} \pm \frac{1}{2} \sqrt{\nu^{2}-4 \tilde{\mu}}
$$


cuja parte real é sempre negativa.

É fácil checar que a função $(B, U)=h(\Omega):=\left(\tilde{\mu}^{-1}\left(-\Omega^{2}+1 / 3 \operatorname{Tr}\left(\Omega^{2}\right) \mathrm{Id}\right), 0\right)$ define uma variedade central em $\Omega=0$. De fato, $h(0)=0$ e $h^{\prime}(0)=0$. Além disso, se tomarmos $\Omega_{0}$ suficientemente pequeno e $\left(B_{0}, U_{0}\right)=h\left(\Omega_{0}\right),\left(\Omega_{0}, B_{0}, U_{0}\right)$ é uma solução de equilíbrio do sistema, portanto esta variedade é invariante. O fluxo restrito é trivial, já que todos seus pontos são equilíbrios do sistema, logo estáveis.

Então, o Teorema 3.21 garante que se $(\Omega(0), B(0), U(0))$ é suficientemente pequeno, existe uma matriz constante $\Omega_{e q}$ e constantes $C_{1}, C_{2}>0$ tais que

$$
\left\|\Omega(t)-\Omega_{e q}\right\| \leq C_{1} e^{-|\operatorname{Re}(\lambda)| t}, \quad\left\|\tilde{\mu} B(t)-\left(-\Omega_{e q}^{2}+\frac{1}{3} \operatorname{Tr}\left(\Omega_{e q}^{2}\right) \operatorname{Id}\right)\right\| \leq C_{2} e^{-|\operatorname{Re}(\lambda)| t} .
$$

Da seção anterior, vemos que $\Omega_{e q}$ é determinado pelo momento angular (3.28), as desigualdades (3.38) fornecem uma estimativa para a velocidade de atração. Observe que esta análise pode ser feita em qualquer equilíbrio (3.24), (3.26). Esta é uma extensão interessante desde que sabemos que soluções com momento angular não-nulo não podem se aproximar arbitrariamente da origem. Portanto, vamos encontrar os autovalores da parte linear de (3.24), (3.26) em um equilíbrio qualquer $\Omega_{0},\left(B_{0}, U_{0}\right)=h\left(\Omega_{0}\right)$.

Faça a reescala de tempo $\tau=\nu$ t. Denotando as derivadas com respeito ao novo tempo por $B^{\prime}, Y^{\prime}$, temos $B^{\prime}=\nu \dot{B}, B^{\prime \prime}=\nu^{2} \ddot{B}, \tilde{\Omega}=\nu \Omega$. Por simplicidade, mantenhamos a mesma notação para $\Omega$. Então, o sistema (3.24), (3.26) fica

$$
\left(\begin{array}{c}
B^{\prime} \\
U^{\prime} \\
\Omega^{\prime}
\end{array}\right)=F(B, U, \Omega)=\left(\begin{array}{c}
U \\
-U-\beta^{2} B-\Omega^{2}+1 / 3 \operatorname{Tr}\left(\Omega^{2}\right) \mathrm{Id} \\
\varepsilon \Phi_{\varepsilon B}^{-1}\left(\left[B, \Omega^{2}\right]-U \Omega-\Omega U\right)
\end{array}\right),
$$

onde $\beta^{2}=\tilde{\mu} / \nu^{2}$.

Suponha que $\lambda$ seja um autovalor da Jacobiana de $F$ no equilíbrio. Assim, temos

$$
\left.\frac{d}{d \eta}\right|_{\eta=0} F\left(B_{0}+\eta B, \eta U, \Omega_{0}+\eta \Omega\right)=D F\left(B_{0}, 0, \Omega_{0}\right)(B, U, \Omega)=\lambda(B, U, \Omega) .
$$

Então,

$$
\begin{gathered}
U=\lambda B, \\
-U-\beta^{2} B-\Omega_{0} \Omega-\Omega \Omega_{0}+\frac{2}{3} \operatorname{Tr}\left(\Omega_{0} \Omega\right) \operatorname{Id}=\lambda U, \\
\left.\varepsilon \frac{d}{d \eta}\right|_{\eta=0} \Phi_{\left(\varepsilon\left(B_{0}+\eta B\right)\right)}^{-1}\left(\left[B_{0}+\eta B,\left(\Omega_{0}+\eta \Omega\right)^{2}\right]-\eta U\left(\Omega_{0}+\eta \Omega\right)-\eta\left(\Omega_{0}+\eta \Omega\right) U\right)=\lambda \Omega .
\end{gathered}
$$

Observando que $\left[B_{0}, \Omega_{0}^{2}\right]=0$, concluímos que (3.40) é equivalente a

$$
\left[B, \Omega_{0}^{2}\right]+\left[B_{0}, \Omega_{0} \Omega\right]+\left[B_{0}, \Omega \Omega_{0}\right]-U \Omega_{0}-\Omega_{0} U=\lambda \varepsilon^{-1} \Phi_{\varepsilon B_{0}}(\Omega)
$$

Logo, o problema é reduzido ao par de equações

$$
\begin{gathered}
-\Omega_{0} \Omega-\Omega \Omega_{0}+\frac{2}{3} \operatorname{Tr}\left(\Omega_{0} \Omega\right) \operatorname{Id}=\left(\lambda^{2}+\lambda+\beta^{2}\right) B \\
{\left[B+\beta^{-2}\left(\Omega_{0} \Omega+\Omega \Omega_{0}\right), \Omega_{0}^{2}\right]-\lambda B \Omega_{0}-\lambda \Omega_{0} B=\lambda\left(\varepsilon^{-1} \Omega-\Omega_{0}^{2} \Omega-\Omega \Omega_{0}^{2}+\frac{2}{3} \operatorname{Tr}\left(\Omega_{0}^{2}\right) \Omega\right) .}
\end{gathered}
$$


Multiplique (3.42) por $\lambda^{2}+\lambda+\beta^{2}$ e use (3.41) para obter

$$
\begin{gathered}
\beta^{-2} \lambda(\lambda+1)\left[\Omega_{0} \Omega+\Omega \Omega_{0}, \Omega_{0}^{2}\right]+\lambda\left(2 \Omega_{0} \Omega \Omega_{0}+\Omega \Omega_{0}^{2}+\Omega_{0}^{2} \Omega-\frac{4}{3} \operatorname{Tr}\left(\Omega_{0} \Omega\right) \Omega_{0}\right)= \\
=\lambda\left(\lambda^{2}+\lambda+\beta^{2}\right)\left(\varepsilon^{-1} \Omega-\Omega_{0}^{2} \Omega-\Omega \Omega_{0}^{2}+\frac{2}{3} \operatorname{Tr}\left(\Omega_{0}^{2}\right) \Omega\right) .
\end{gathered}
$$

Fixando $S\left(\Omega_{0}\right)=\left(\omega_{0}, 0,0\right)$, esta equação fica equivalente ao sistema

$$
\begin{gathered}
\lambda \omega_{1}\left(4 \omega_{0}^{2}+\left(\lambda^{2}+\lambda+\beta^{2}\right)\left(3 \varepsilon^{-1}-2 \omega_{0}^{2}\right)\right)=0, \\
3 \omega_{0}^{3} \omega_{3} \beta^{-2} \lambda(\lambda+1)+\lambda \omega_{2}\left(3 \omega_{0}^{2}+\left(\lambda^{2}+\lambda+\beta^{2}\right)\left(3 \varepsilon^{-1}-\omega_{0}^{2}\right)\right)=0, \\
3 \omega_{0}^{3} \omega_{2} \beta^{-2} \lambda(\lambda+1)-\lambda \omega_{3}\left(3 \omega_{0}^{2}+\left(\lambda^{2}+\lambda+\beta^{2}\right)\left(3 \varepsilon^{-1}-\omega_{0}^{2}\right)\right)=0 .
\end{gathered}
$$

Note que as equações (3.44) e (3.45) implicam em

$$
\begin{gathered}
\lambda \omega_{0}^{3}\left(\omega_{2}^{2}+\omega_{3}^{2}\right)(\lambda+1)=0, \\
\lambda\left(\omega_{2}^{2}+\omega_{3}^{2}\right)\left(3 \omega_{0}^{2}+\left(\lambda^{2}+\lambda+\beta^{2}\right)\left(3 \varepsilon^{-1}-\omega_{0}^{2}\right)\right)=0 .
\end{gathered}
$$

As soluções deste problema devem ser analisadas caso a caso. Note que o caso $\omega_{0}=0$ já foi considerado.

(i) Núcleo da Jacobiana $(\lambda=0)$. De (3.41)

$$
\beta^{2} B+\Omega \Omega_{0}+\Omega_{0} \Omega=\frac{2}{3} \operatorname{Tr}\left(\Omega_{0} \Omega\right) \mathrm{Id},
$$

que comuta com $\Omega_{0}^{2}$, e portanto satisfaz (3.42). Logo, o núcleo é gerado pelos vetores

$$
(B, U, \Omega)=\left(-\frac{\omega_{0}}{3}\left(\begin{array}{ccc}
4 \omega_{1} & 3 \omega_{2} & 3 \omega_{3} \\
3 \omega_{2} & -2 \omega_{1} & 0 \\
3 \omega_{3} & 0 & -2 \omega_{1}
\end{array}\right), 0, \Omega\right), \quad \forall \Omega \in \operatorname{skew}(3)
$$

logo é 3-dimensional. Note que este é exatamente o espaço tangente à variedade central, $(B, U)=h(\Omega)$, no equilíbrio escolhido.

(ii) Suponha $\lambda^{2}+\lambda+\beta^{2}=0$ (hiperbólico estável).

De (3.41) vemos que

$$
\frac{\omega_{0}}{3}\left(\begin{array}{ccc}
4 \omega_{1} & 3 \omega_{2} & 3 \omega_{3} \\
3 \omega_{2} & -2 \omega_{1} & 0 \\
3 \omega_{3} & 0 & -2 \omega_{1}
\end{array}\right)=0 \quad \Rightarrow \Omega=0
$$

Assim, de (3.42)

$$
\left(\begin{array}{ccc}
0 & b_{12} & b_{13} \\
-b_{12} & 0 & 0 \\
-b_{13} & 0 & 0
\end{array}\right)=\lambda\left(\begin{array}{ccc}
0 & b_{13} & -b_{12} \\
-b_{13} & 0 & -b_{22}-b_{33} \\
b_{12} & b_{22}+b_{33} & 0
\end{array}\right) .
$$

O auto-espaço associado é 2-dimensional.

(iii) Suponha $\lambda^{2}+\lambda+\beta^{2} \neq 0$ e $\lambda \neq 0$. 
Supondo $\omega_{2}^{2}+\omega_{3}^{2} \neq 0,(3.46)$ e (3.47) implicam que $3 \varepsilon^{-1}+2 \omega_{0}^{2}=0$, uma contradição. Logo, $\omega_{2}^{2}+\omega_{3}^{2}=0$. Se um deles for nulo, o outro também será, então $\omega_{1} \neq 0$. Por (3.43), isto é possível somente se $3 \varepsilon^{-1}-2 \omega_{0}^{2} \neq 0$, e o autovalor $\lambda$ deve resolver

$$
\lambda^{2}+\lambda+\frac{3 \beta^{2}+2 \varepsilon \omega_{0}^{2}\left(2 \beta^{2}-1\right)}{3-2 \varepsilon \omega_{0}^{2}}=0 .
$$

Então, garantimos que as partes reais desses autovalores são negativas se $\omega_{0}^{2}<3 /(2 \varepsilon)$ e $2 \beta^{2} \geq 1$. O auto-espaço correspondente também é 2-dimensional.

Se $\omega_{2} \neq 0$, tome $\omega_{2}=\omega$ e $\omega_{3}= \pm i \omega$. Suponha $\omega_{1} \neq 0$. Então, por (3.43) segue que

$$
\lambda^{2}+\lambda=-\frac{3 \beta^{2}+2 \varepsilon \omega_{0}^{2}\left(2 \beta^{2}-1\right)}{3-2 \varepsilon \omega_{0}^{2}}=:-b \in \mathbb{R} .
$$

Mas, de (3.44) concluímos que $\lambda$ é imaginário puro, e isto é incompatível com a equação acima. Portanto, $\omega_{1}=0$. Logo, novamente de (3.44), temos

$$
\lambda^{2}+\left(1 \pm i \frac{3 \omega_{0}^{3} \varepsilon \beta^{-2}}{3-\omega_{0}^{2} \varepsilon}\right) \lambda+\frac{3 \beta^{2}+\omega_{0}^{2} \varepsilon\left(3-\beta^{2}\right) \pm 3 i \omega_{0}^{3} \varepsilon \beta^{-2}}{3-\omega_{0}^{2} \varepsilon}=0
$$

Observe que as raízes de um polinômio de grau 2 dependem continuamente de seus coeficientes (caso possuam multiplicidade 1). Logo, concluímos que a parte real de ambas as raízes deste polinômio são negativas, se $\omega_{0}^{2} \varepsilon$ é suficientemente pequeno.

Em resumo, temos somente autovalores de parte real negativa. Pelo Teorema 1, Seção 2.12, de [Per01] vemos que neste caso, em coordenadas adequadas, o sistema tem a forma (3.35). Podemos assim aplicar o mesmo resultado, adaptando a coordenada coordenada $x=\left(\Omega, D h_{\Omega_{0}}(\Omega)\right)$. Para determinar o fator $\sigma$, observe que alguns dos autovalores têm parte real $-1 / 2$, e os demais são solução de (3.49). Assim, é fácil checar que

$$
\sigma_{0}=\frac{1}{2}-\frac{1}{\sqrt{2}} \sqrt{\sqrt{\left(a^{2}+4 b-1\right)^{2}+4 a^{2}}-\left(a^{2}+4 b-1\right)}<\frac{1}{2},
$$

onde

$$
a:=\frac{3 \omega_{0}^{3} \varepsilon \beta^{-2}}{3-\omega_{0}^{2} \varepsilon}, \quad b:=\frac{3 \omega_{0}^{2} \varepsilon}{3-\omega_{0}^{2} \varepsilon}+\beta^{2} .
$$

Novamente, pelo Teorema 3.21, e revertendo a escala de tempo, concluímos que para toda condição inicial próxima à variedade central, existe $\Omega_{e q}$ tal que,

$$
\left\|\Omega(t)-\Omega_{e q}\right\| \leq\left\|x(t)-x_{e q}\right\| \leq C e^{-\sigma_{0} \tau}=C e^{-\sigma_{0} \nu t},
$$

onde a constante $\omega_{0}$ pode ser obtida em termos do momento angular, por (3.29), o qual é fixo.

\subsection{Problema de dois corpos}

Estendemos a discussão da Seção 3.2 propondo a interação do corpo pseudo-rígido com um segundo corpo. Supomos que o raio deste seja muito menor que a distância que os separa, considerando-o como pontual, ou seja, desprezando o movimento de suas partes internas. Assumamos que a interação entre os dois seja somente gravitacional, Newtoniana, e que sua intensidade seja fraca o suficiente para que a deformação não viole as condições do Lema 3.18. A função dissipação será a mesma definida em (3.23).

Precisamos introduzir uma escala adequada para a posição relativa entre os corpos. Sabemos que o campo de forças que atua sobre o segundo corpo é somente o gradiente do potencial de interação. Sejam $m>0$ e $\vec{p} \in \mathbb{R}^{3}$ a massa e a posição do segundo corpo, $\vec{P} \in \mathbb{R}^{3}$ a posição do centro de massa 
primeiro corpo. As demais coordenadas são as mesmas da Seção 3.2. O potencial de interação é dado por

$$
V_{\text {int }}=-G m \rho \int_{\mathcal{B}} \frac{1}{\left\|Y^{T}(\vec{p}-\vec{P})-e^{\varepsilon B} x\right\|} d x=-G m \rho \int_{\mathcal{B}} \frac{1}{\left\|Y^{T}(\vec{r})-e^{\varepsilon B} x\right\|} d x,
$$

onde $\vec{r}:=\vec{p}-\vec{P}$ é a posição relativa entre os corpos. A equação a ele associada é

$$
\tilde{m} \ddot{\vec{r}}=-\nabla_{\vec{r}} V_{i n t},
$$

sendo $\tilde{m}$ a massa reduzida do sistema.

Também vamos aproximar este potencial para pequenas deformações usando novamente as simetrias da integral que o define

$$
\begin{gathered}
V_{\text {int }}(Y, B, \vec{r})=:-G m \rho \phi_{2}(\varepsilon B), \\
\phi_{2}(\varepsilon B)=\phi_{2}(0)+\varepsilon D \phi(0) B+\mathcal{O}\left(\varepsilon^{2}\right)=\int_{\mathcal{B}} \frac{1}{\|\vec{r}-Y x\|} d x+\varepsilon \int_{\mathcal{B}} \frac{\left\langle Y^{T} \vec{r}-x, B x\right\rangle}{\left\|Y^{T} \vec{r}-x\right\|^{3}} d x+\mathcal{O}\left(\varepsilon^{2}\right) .
\end{gathered}
$$

Mas, note que usando a notação de vetores como matrizes coluna

$$
\int_{\mathcal{B}} \frac{\left\langle\vec{r}-x, Y B Y^{T} x\right\rangle}{\|\vec{r}-x\|^{3}} d x=\operatorname{Tr}\left(Y B Y^{T} \int_{\mathcal{B}} \frac{x(\vec{r}-x)^{T}}{\|\vec{r}-x\|^{3}} d x\right) .
$$

E pelo Apêndice C, equação (C.1),

$$
\int_{\mathcal{B}} \frac{x(\vec{r}-x)^{T}}{\|\vec{r}-x\|^{3}} d x=g_{1}(\vec{r}) \operatorname{Id}+\frac{4 \pi R^{5}}{5 r^{5}} \vec{r} \cdot \vec{r}^{T},
$$

portanto,

$$
V_{\text {int }}(Y, B, \vec{r})=-\frac{G m M}{\|\vec{r}\|}-\frac{3 G m M R^{2}}{5 r^{5}} \varepsilon \operatorname{Tr}\left(\left(Y^{T} \vec{r}\right)\left(Y^{T} \vec{r}\right)^{T} B\right)+\mathcal{O}\left(\varepsilon^{2}\right) .
$$

Assumindo rotação estacionária em torno do eixo $z$, com velocidade angular $\sqrt{\varepsilon} \omega$ e rotação orbital síncrona, $\vec{r}(t)=r_{0}\left(\cos \left(\varepsilon^{\frac{1}{2}} \omega t\right)\right.$, sen $\left.\left(\varepsilon^{\frac{1}{2}} \omega t\right)\right)$, a equação (3.52) implica que a constante $r_{0}$ deve ser solução da equação:

$$
-\omega^{2} \varepsilon=-G M r_{0}^{-3}+\alpha \varepsilon r_{0}^{-5} \quad \Rightarrow \quad r_{0}^{-3}=\mathcal{O}(\varepsilon) .
$$

Esta equação impõe uma mudança de escala para o vetor posição: $\vec{r}=\varepsilon^{-\frac{1}{3}} \tilde{\vec{r}}$ (novamente não mudaremos a notação, por simplicidade).

Logo, a energia potencial total fica:

$V(Y, B, \vec{r}):=\left(\frac{4 \pi \mu R^{3}}{3}+\frac{4 G M^{2}}{25 R}\right) \varepsilon^{2} \operatorname{Tr}\left(B^{2}\right)-\frac{G m M}{\|\vec{r}\|} \varepsilon^{\frac{1}{3}}-\frac{3 G m M R^{2}}{5 r^{5}} \varepsilon^{2} \operatorname{Tr}\left(\left(Y^{T} \vec{r}\right)\left(Y^{T} \vec{r}\right)^{T} B\right)+\mathcal{O}\left(\varepsilon^{\frac{5}{2}}\right)$.

A energia cinética do pseudo-rígido sofre uma pequena alteração

$$
\begin{gathered}
T_{1}=\frac{1}{2} \int_{\mathcal{B}} \rho(\|x\|)\left\|\sqrt{\varepsilon} \Omega A x+\dot{A} x+Y^{T} \dot{\vec{P}}\right\|^{2} d x= \\
=\frac{M}{2} \dot{\vec{P}}^{2}+\frac{1}{2} \int_{\mathcal{B}} \rho(\|x\|)\left(\varepsilon\|\Omega A x\|^{2}+\|\dot{A} x\|^{2}+2 \sqrt{\varepsilon}\langle x, \dot{A} \Omega A x\rangle+\left\langle(\sqrt{\varepsilon} \Omega A+\dot{A}) x, Y^{T} \dot{\vec{P}}\right\rangle\right) d x
\end{gathered}
$$

Observe que o último termo da integral é uma função ímpar, portanto sua integral é nula. 
Expandindo a integral em Taylor até ordem $\varepsilon^{\frac{5}{2}}$ obtemos a energia cinética total

$$
T(\Omega, B, \vec{p}, \vec{P}, \dot{B}, \dot{\vec{p}}, \dot{\vec{P}})=\frac{\mathrm{I}_{\circ}}{4}\left(\varepsilon^{2} \operatorname{Tr}\left(\dot{B}^{2}\right)-\varepsilon \operatorname{Tr}\left(\Omega^{2}\right)-2 \varepsilon^{2} \operatorname{Tr}\left(\Omega^{2} B\right)\right)+\frac{M}{2} \dot{\vec{P}}^{2}+\frac{1}{2} m \dot{\vec{p}}^{2} .
$$

Considere a mudança de coordenadas

$$
\vec{r}:=\varepsilon^{\frac{1}{3}}(\vec{p}-\vec{P}), \quad \vec{R}_{C M}:=\varepsilon^{\frac{1}{3}} \frac{m \vec{p}+M \vec{P}}{m+M} .
$$

Desta forma, multiplicando a Lagrangiana por $\varepsilon^{\frac{2}{3}}$ e desprezando seus termos de ordem maior que $\varepsilon^{\frac{8}{3}}$, temos

$$
\begin{gathered}
\varepsilon^{\frac{2}{3}} \mathcal{L}=\frac{\mathrm{I}_{\circ}}{4}\left(\varepsilon^{\frac{8}{3}} \operatorname{Tr}\left(\dot{B}^{2}\right)-\varepsilon^{\frac{5}{3}} \operatorname{Tr}\left(\Omega^{2}\right)-2 \varepsilon^{\frac{8}{3}} \operatorname{Tr}\left(\Omega^{2} B\right)-\tilde{\mu} \varepsilon^{\frac{8}{3}} \operatorname{Tr}\left(B^{2}\right)\right)+\frac{1}{2} \tilde{m} \dot{\vec{r}}^{2}+ \\
+\frac{\tilde{M}}{2} \dot{\vec{R}}_{C M}^{2}+\frac{G m M}{\|\vec{r}\|} \varepsilon+\frac{3 G m M R^{2}}{5 r^{5}} \varepsilon^{\frac{8}{3}} \operatorname{Tr}\left(\left(Y^{T} \vec{r}\right)\left(Y^{T} \vec{r}\right)^{T} B\right),
\end{gathered}
$$

onde $\tilde{M}:=M+m$ e $\tilde{m}=m M /(M+m)$ são a massa total e a massa reduzida do sistema, respectivamente.

Observamos que, como usual, podemos tomar a posição do centro de massa fixa no espaço. Reforçamos que daqui em diante o corpo pseudo-rígido será considerado homogêneo.

Assim, usando as mesmas ideias da Seção 3.2, obtemos as equações de movimento (em relação a $B)$

$$
\ddot{B}+\nu \dot{B}+\tilde{\mu} B=-\Omega^{2}+\frac{1}{3} \operatorname{Tr}\left(\Omega^{2}\right) \operatorname{Id}+\frac{3 G m}{r^{5}}\left(\left(Y^{T} \vec{r}\right)\left(Y^{T} \vec{r}\right)^{T}-\frac{1}{3} r^{2} \mathrm{Id}\right) .
$$

Usando novamente o Lema 2.6, obtemos equações correspondentes à parte rotacional usando a expressão

$$
\frac{d}{d t}\left(\left(\frac{\partial T}{\partial \Omega}\right)_{a}\right)+\left[\Omega,\left(\frac{\partial T}{\partial \Omega}\right)_{a}\right]=-Y^{T} P_{Y}\left(\frac{\partial V}{\partial Y}\right)
$$

onde os gradientes indicados são calculados segundo a métrica Euclidiana, e $P_{Y}(H):=Y\left(Y^{T} H\right)_{a}$ é a projeção ortogonal deduzida no Lema 2.5. Mas, note que

$$
\begin{gathered}
D V_{Y}(H)=-\frac{8}{5} G m \pi \rho \varepsilon^{2} \frac{R^{5}}{r^{5}} \operatorname{Tr}\left(B Y^{T} \vec{r} \cdot \vec{r}^{T} H\right) \\
\Rightarrow \frac{\partial V}{\partial Y}=-\frac{6}{5} G m M \varepsilon^{2} \frac{R^{2}}{r^{5}} \vec{r} \cdot \vec{r}^{T} Y B \Rightarrow Y^{T} P_{Y}\left(\frac{\partial V}{\partial Y}\right)=-\frac{3 G m M \varepsilon^{2} R^{2}}{5 r^{5}}\left(Y^{T} \vec{r} \cdot \vec{r}^{T} Y B-B Y^{T} \vec{r} \cdot \vec{r}^{T} Y\right) .
\end{gathered}
$$

Assim, usando os cálculos da Seção 3.2, obtemos as equações de movimento

$$
\Phi_{\varepsilon B}(\dot{\Omega})+\varepsilon(\Omega \dot{B}+\dot{B} \Omega)=\varepsilon\left[B, \Omega^{2}\right]+\frac{3 G m}{r^{5}} \varepsilon\left[\left(Y^{T} \vec{r}\right)\left(Y^{T} \vec{r}\right)^{T}, B\right] .
$$

Agora note que

$$
\begin{gathered}
D V_{\vec{r}}(\vec{h})=\frac{G m M}{r^{3}} \varepsilon^{\frac{1}{3}}<\vec{r}, \vec{h}>-\frac{6 G m M \varepsilon^{2} R^{2}}{5 r^{5}} \operatorname{Tr}\left(\left(Y B Y^{T} \vec{r}\right)(\vec{h})^{T}\right)+\frac{3 G m M \varepsilon^{2} R^{2}}{r^{7}} \operatorname{Tr}\left(Y B Y^{T}(\vec{r})(\vec{r})^{T}\right)<\vec{r}, \vec{h}> \\
=\frac{G m M}{r^{3}} \varepsilon^{\frac{1}{3}}<\vec{r}, \vec{h}>-\frac{6 G m M \varepsilon^{2} R^{2}}{5 r^{5}}\left\langle Y B Y^{T} \vec{r}, \vec{h}\right\rangle+\frac{3 G m M \varepsilon^{2} R^{2}}{r^{7}}\left\langle Y B Y^{T} \vec{r}, \vec{r}\right\rangle<\vec{r}, \vec{h}>.
\end{gathered}
$$

Logo, as equações de movimento para $\vec{r}$ são:

$$
\ddot{\vec{r}}=-\frac{G \tilde{M} \varepsilon}{r^{3}} \vec{r}-\frac{3 G \tilde{M} \varepsilon^{\frac{8}{3}} R^{2}}{r^{7}}\left\langle Y B Y^{T} \vec{r}, \vec{r}\right\rangle \vec{r}+\frac{6 G \tilde{M} \varepsilon^{\frac{8}{3}} R^{2}}{5 r^{5}} Y B Y^{T} \vec{r} .
$$


Do ponto de vista observacional, as quantidades interessantes neste problema são $\Omega$ e $\vec{r}$. Adotemos um procedimento que permita-nos substituir a dependência da deformação nas equações por sua média temporal. A ideia heurística é eliminar a dependência das oscilações no movimento, levando em conta somente o amortecimento. Para isto, usaremos os resultados da Teoria da Perturbação Singular, listados na Seção 2.3.2.

Considerando todos os graus de liberdade mencionados, o movimento é descrito pelo sistema de equações $(3.57),(3.59)$ e (3.60). Escrevendo em forma de sistema de primeira ordem, temos

$$
\begin{aligned}
& \dot{B}=U \\
& \dot{U}=-\nu U-\tilde{\mu} B+F(Y, \Omega, \vec{r}) \\
& \dot{\Omega}=\varepsilon G(Y, \Omega, B, U, \vec{r}, \varepsilon) \\
& \dot{\vec{r}}=\sqrt{\varepsilon} \vec{v} \\
& \dot{\vec{v}}=\sqrt{\varepsilon} h(Y, B, \vec{r}) \\
& \dot{Y}=\sqrt{\varepsilon} Y \Omega
\end{aligned}
$$

observando que a função $F(Y, \Omega, \vec{r})$ é quadrática em todas estas variáveis. Observe que este é um sistema lento-rápido na escala $\sqrt{\varepsilon}$, no tempo rápido. O sistema lento associado é, fazendo-se $t=\sqrt{\varepsilon} \tau$,

$$
\begin{aligned}
& \sqrt{\varepsilon} B^{\prime}=U \\
& \sqrt{\varepsilon} U^{\prime}=-\nu U-\tilde{\mu} B+F(Y, \Omega, \vec{r}) \\
& \Omega^{\prime}=\sqrt{\varepsilon} G(Y, \Omega, B, U, \vec{r}, \varepsilon) \\
& \vec{r}^{\prime}=\vec{v} \\
& \vec{v}^{\prime}=h(Y, B, \vec{r}) \\
& Y^{\prime}=Y \Omega
\end{aligned}
$$

Portanto, usando a notação da Seção 2.3.2, a variável rápida é $x=(B, U)$ e a variável lenta é $y=(Y, \Omega, \vec{r}, \vec{v})$. A variedade lenta é dada por

$$
0=U, \quad 0=-\nu U-\tilde{\mu} B+F(Y, \Omega, \vec{r}),
$$

portanto, sua parametrização global é $x=(B, U)=x^{\star}(y)=\left(\tilde{\mu}^{-1} F(Y, \Omega, \vec{r}), 0\right), \forall(Y, \Omega, \vec{r}, \vec{v})$.

Observe que $\left[F, \Omega^{2}\right]+3 G m r^{-5}\left[\left(Y^{T} \vec{r}\right)\left(Y^{T} \vec{r}\right)^{T}, F\right]=0$, e

$$
Y F Y^{T} \vec{r}=\tilde{\mu}^{-1}\left(\frac{2 G m}{r^{3}} \vec{r}-\langle\tilde{\omega}, \vec{r}\rangle \tilde{\omega}+\frac{2}{3} \tilde{\omega}^{2} \vec{r}\right)
$$

onde $\tilde{\omega}=S\left(Y \Omega Y^{T}\right)$ é a velocidade angular do corpo no referencial inercial.

Assim, a média lenta, definida pela equação (2.38), fica

$$
\begin{gathered}
\Omega^{\prime}+\frac{\varepsilon}{\tilde{\mu}}\left(F(Y, \Omega, \vec{r}) \Omega^{\prime}+\Omega^{\prime} F(Y, \Omega, \vec{r})\right)=0, \\
\vec{r}^{\prime \prime}=-\frac{G \tilde{M}}{r^{3}} \vec{r}-\frac{6 G \tilde{M} R^{2} \varepsilon^{\frac{5}{3}}}{5 \tilde{\mu} r^{7}}\left(\left(\frac{3 G m}{r}+\frac{r^{2} \tilde{\omega}^{2}}{2}-\frac{5}{2}<\vec{r}, \tilde{\omega}>^{2}\right) \vec{r}+r^{2}<\tilde{\omega}, \vec{r}>\tilde{\omega}\right) .
\end{gathered}
$$

Observe que, pelo Lema 3.18 , no aberto $\|F(Y, \Omega, \vec{r})\|<\tilde{\mu} / 2 \varepsilon$, a primeira equação implica que $\Omega(\tau)=\Omega_{0}$, constante. Portanto, o vetor $\tilde{\omega}$ em (3.63) é fixo, nesta primeira aproximação. É evidente que o termo adicional que surge nesta equação é a atração gravitacional exercida pela maré induzida no corpo deformável, devido tanto a sua rotação quanto pela própria força gravitacional.

Passemos agora para uma melhor aproximação da variedade adiabática, citada no Teorema 2.40, usando sua expansão de primeira ordem, em $\sqrt{\varepsilon}$, de (2.43). Para tanto, note que dadas duas matrizes $H_{1}, H_{2} \in \operatorname{Sym}(3), \operatorname{Tr}\left(H_{i}\right)=0$,

$$
A^{\star}(y)\left(H_{1}, H_{2}\right)=\frac{\partial f}{\partial x}\left(x^{\star}(y), y, \varepsilon\right)\left(H_{1}, H_{2}\right)=
$$




$$
=\left.\frac{d}{d \lambda}\right|_{\lambda=0}\left(\lambda H_{2},-\nu\left(\lambda H_{2}\right)-F(Y, \Omega, \vec{r})+\tilde{\mu}\left(\lambda H_{1}\right)+\tilde{\mu}^{-1} F(Y, \Omega, \vec{r})\right)=\left(H_{2},-\nu H_{2}-\tilde{\mu} H_{1}\right),
$$

$\log$,

$$
A^{\star}(y)^{-2}\left(H_{1}, H_{2}\right)=\tilde{\mu}^{-2}\left(\nu H_{2}+\left(\nu^{2}-\tilde{\mu}\right) H_{1},-\tilde{\mu}\left(H_{2}+\nu H_{1}\right)\right) .
$$

Dadas $M_{1} \in \operatorname{skew}(3), M_{2} \in \mathrm{SO}(3), h_{1}, h_{2} \in \mathbb{R}^{3}$,

$$
\frac{\partial f}{\partial y}\left(x^{\star}(y), y\right)\left(M_{1}, M_{2}, h_{1}, h_{2}\right)=\tilde{\mu}^{-1}\left(0, \frac{\partial F}{\partial \Omega} M_{1}+\frac{\partial F}{\partial Y} M_{2}+\frac{\partial F}{\partial \vec{r}} h_{1}\right),
$$

$\log \mathrm{O}$

$$
\frac{\partial f}{\partial y}\left(x^{\star}(y), y\right) g\left(x^{\star}(y), y\right)=\tilde{\mu}^{-1}\left(0, \sqrt{\varepsilon} \frac{\partial F}{\partial \Omega} G\left(Y, \Omega, \tilde{\mu}^{-1} F, 0, \vec{r}, \varepsilon\right)+\frac{\partial F}{\partial Y} Y \Omega+\frac{\partial F}{\partial \vec{r}} \vec{v}\right) .
$$

Lembre que $G\left(Y, \Omega, \tilde{\mu}^{-1} F, 0, \vec{r}, \varepsilon\right)=0$. Ainda,

$$
\begin{gathered}
\frac{\partial F}{\partial Y} Y \Omega=-\frac{3 G m}{r^{5}}\left(\left(\Omega Y^{T} \vec{r}\right)\left(Y^{T} \vec{r}\right)^{T}+\left(Y^{T} \vec{r}\right)\left(\Omega Y^{T} \vec{r}\right)^{T}\right) \\
\frac{\partial F}{\partial \vec{r}} \vec{v}=\frac{3 G m}{r^{5}}\left(-\frac{5}{r^{2}}\langle\vec{r}, \vec{v}\rangle\left(Y^{T} \vec{r}\right)\left(Y^{T} \vec{r}\right)^{T}+\left(Y^{T} \vec{v}\right)\left(Y^{T} \vec{r}\right)^{T}+\left(Y^{T} \vec{r}\right)\left(Y^{T} \vec{v}\right)^{T}+\langle\vec{r}, \vec{v}\rangle \mathrm{Id}\right) .
\end{gathered}
$$

Portanto,

$$
\begin{gathered}
(B, U)=x^{\star}(y)-\sqrt{\varepsilon} A^{\star}(y)^{-2} \frac{\partial f}{\partial y}\left(x^{\star}(y), y\right) g\left(x^{\star}(y), y\right)+\mathcal{O}\left(\sqrt{\varepsilon}^{2}\right)= \\
=\left(\tilde{\mu}^{-1} F-\frac{\nu \sqrt{\varepsilon}}{\tilde{\mu}^{2}}\left(\frac{\partial F}{\partial Y} Y \Omega+\frac{\partial F}{\partial \vec{r}} \vec{v}\right), \frac{\sqrt{\varepsilon}}{\tilde{\mu}}\left(\frac{\partial F}{\partial Y} Y \Omega+\frac{\partial F}{\partial \vec{r}} \vec{v}\right)\right)+\mathcal{O}\left(\sqrt{\varepsilon}^{2}\right) .
\end{gathered}
$$

Desta forma, com esta correção, o sistema reduzido associado a (3.63) ganha o termo adicional

$$
\vec{T}=-\frac{18 G^{2} \tilde{M} m R^{2} \varepsilon^{\frac{13}{6}} \nu}{5 \tilde{\mu}^{2} r^{8}}\left(\dot{\vec{r}}+\vec{r} \times \tilde{\omega}+2 \frac{<\vec{r}, \dot{\vec{r}}>}{r^{2}} \vec{r}\right) .
$$

Lembre que o Teorema 2.42 garante a aproximação assintótica entre as órbitas dos sistemas (3.62) e (3.63), em $\mathcal{O}(\sqrt{\varepsilon})$. Para isto, é preciso garantir que a variedade lenta é uniformemente assintoticamente estável. Mas, de (3.64), vemos que se $\lambda$ é autovalor de $A^{\star}(y)$,

$$
A^{\star}(y)\left(H_{1}, H_{2}\right)=\left(H_{2},-\nu H_{2}-\tilde{\mu} H_{1}\right)=\lambda\left(H_{1}, H_{2}\right),
$$

de onde concluímos que $\lambda$ é solução de

$$
\lambda^{2}+\nu \lambda+\tilde{\mu}=0,
$$

logo, sua parte real é sempre negativa, uniformemente em $y$, garantindo o que pretendíamos.

Fazendo hipóteses adicionais sobre o sistema, assumimos que o movimento do satélite está restrito ao plano ortogonal à direção da velocidade angular $\tilde{\omega}$. Neste caso, a equação de movimento para a órbita se reduz a

$$
\vec{r}^{\prime \prime}=-\frac{G \tilde{M}}{r^{3}} \vec{r}-\frac{3 G \tilde{M} R^{2} \varepsilon^{\frac{5}{3}}}{5 \tilde{\mu} r^{5}} \omega^{2} \vec{r}-\frac{3 G m R^{5} \varepsilon^{\frac{13}{6}}}{r^{8}}\left(\frac{6 G \tilde{M}}{5 \tilde{\mu} R^{3}}\right)\left(\vec{r}+\frac{\nu}{\tilde{\mu}}\left(\dot{\vec{r}}+\vec{r} \times \tilde{\omega}+2 \frac{<\vec{r}, \dot{\vec{r}}>}{r^{2}} \vec{r}\right)\right) .
$$

Podemos comparar esta expressão com as fórmulas utilizadas para tratar o problema de marés. 
Por exemplo, temos a equação (20) de [EL07]

$$
\ddot{\vec{r}}=-\frac{G \tilde{M}}{r^{3}} \vec{r}-\frac{3 k_{2} G m \tilde{M} R^{5}}{M r^{8}}\left(\vec{r}+\Delta t\left(\dot{\vec{r}}+\vec{r} \times \tilde{\omega}+2 \frac{<\vec{r}, \dot{\vec{r}}>}{r^{2}} \vec{r}\right)\right),
$$

onde $k_{2}$ é o segundo número de Love associado ao corpo deformável, e $\Delta t$ é o tempo de atraso na resposta do corpo viscoso à interação gravitacional. Note que o segundo termo da força corresponde à atração gravitacional exercida pela porção do corpo deformada por sua própria rotação, a qual é aparentemente desprezada na análise. Desta forma, o dado modelo indica que

$$
\Delta t=\frac{\nu}{\tilde{\mu}}=\frac{\eta}{\mu+\frac{2}{25} g \rho R}, \quad k_{2}=\frac{6 G M}{5 \tilde{\mu} R^{3}}=\frac{3}{2} \frac{1}{\left(1+\frac{25}{2} \frac{\mu}{\rho g R}\right)} .
$$

Este valor para o número de Love está em conformidade com o obtido por outros métodos (ver equações $(2.1 .9$ a,b) de [Lam80])

$$
k_{2}=\frac{3}{2} \frac{1}{\left(1+\frac{19}{2} \frac{\mu}{\rho g R}\right)} .
$$

Da mesma maneira, podemos obter a média lenta da equação de movimento (3.59), associada à rotação. Usando a substituição (3.65), um cálculo longo mas direto implica em

$$
\begin{aligned}
& \tilde{\Omega}^{\prime}+\varepsilon\left\{\frac { 3 G m \nu \sqrt { \varepsilon } } { \tilde { \mu } r ^ { 5 } } \left[\left(\left(\tilde{\Omega} \vec{r}-\vec{v}+\frac{5}{r^{2}}\langle\vec{r}, \vec{v}\rangle \vec{r}\right) \vec{r}^{T}+\vec{r}(\tilde{\Omega} \vec{r}-\vec{v})^{T}\right) \tilde{\Omega}^{\prime}\right.\right. \\
& \left.+\tilde{\Omega}^{\prime}\left(\left(\tilde{\Omega} \vec{r}-\vec{v}+\frac{5}{r^{2}}\langle\vec{r}, \vec{v}\rangle \vec{r}\right) \vec{r}^{T}+\vec{r}(\tilde{\Omega} \vec{r}-\vec{v})^{T}\right)-2\langle\vec{r}, \vec{v}\rangle \tilde{\Omega}^{\prime}\right]+\frac{1}{\tilde{\mu}}\left[-\tilde{\Omega}^{2} \tilde{\Omega}^{\prime}-\tilde{\Omega}^{\prime} \tilde{\Omega}^{2}+\frac{2}{3} \operatorname{Tr}\left(\tilde{\Omega}^{2}\right) \tilde{\Omega}^{\prime}\right. \\
& \left.\left.\quad+\frac{3 G m}{r^{5}}\left(\tilde{\Omega}^{\prime} \vec{r} \vec{r}^{T}+\vec{r} \vec{r}^{T} \tilde{\Omega}^{\prime}-\frac{2}{3} r^{2} \tilde{\Omega}^{\prime}\right)\right]\right\}=\frac{3 G m \sqrt{\varepsilon}}{\tilde{\mu} r^{5}}\left\{\left[\left(-\vec{v}+\frac{5}{r^{2}}\langle\vec{r}, \vec{v}\rangle \vec{r}\right) \vec{r}^{T}-\vec{r}(\tilde{\Omega} \vec{r}-\vec{v})^{T}\right] \tilde{\Omega}\right. \\
& \left.\quad+\tilde{\Omega}\left[\left(-\vec{v}+\frac{5}{r^{2}}\langle\vec{r}, \vec{v}\rangle \vec{r}\right) \vec{r}^{T}-\vec{r}(\tilde{\Omega} \vec{r}-\vec{v})^{T}\right]^{T}-2\langle\vec{r}, \vec{v}\rangle \tilde{\Omega}\right\}+\frac{3 G m \nu \sqrt{\varepsilon}}{\tilde{\mu}^{2} r^{5}}\left\{\tilde{\Omega} \vec{r} \vec{r}^{T} \tilde{\Omega}^{2}+\tilde{\Omega}^{2} \vec{r} \vec{r}^{T} \tilde{\Omega}\right. \\
& \left.-\vec{r} \vec{r}^{T} \tilde{\Omega}^{3}-\tilde{\Omega}^{3} \vec{r} \vec{r}^{T}+\frac{5}{r^{2}}\langle\vec{r}, \vec{v}\rangle\left[\vec{r} \vec{r}^{T}, \tilde{\Omega}^{2}\right]+\left[\tilde{\Omega}^{2}, \vec{r} \vec{v}^{T}+\vec{v} \vec{r}^{T}\right]\right\}-\frac{(3 G m)^{2} \nu \sqrt{\varepsilon}}{\tilde{\mu}^{2} r^{8}}\left((\tilde{\Omega} \vec{r}-\vec{v}) \vec{r}^{T}-\vec{r}(\tilde{\Omega} \vec{r}-\vec{v})^{T}\right)
\end{aligned}
$$

onde $\tilde{\Omega}$ é a velocidade angular no referencial inercial.

Restrinja a trajetória do corpo pontual a um movimento circular uniforme, $\vec{r}(t)=r_{0}(\cos (\sigma t)$, sen $(\sigma t), 0)$, e $S(\tilde{\Omega})=(0,0, \omega(t))$. Impondo tais restrições, a equação (3.71) se torna muito simples

$$
\omega^{\prime}(t)=-\frac{9 G^{2} m^{2} \nu \sqrt{\varepsilon}}{\tilde{\mu} r_{0}^{6}} \frac{\omega-\sigma}{\tilde{\mu}+\varepsilon\left(\frac{G m}{r_{0}^{3}}+\frac{2}{3} \omega^{2}\right)}
$$

na qual todas soluções convergem para $\sigma$, se $t \rightarrow+\infty$.

\subsubsection{Número de Love dependente de frequência e o tempo de atraso}

Chamamos a atenção para a equação (3) da referência [CL03]. Apresenta-se um número de Love dependente da frequência de excitação do material, afirmando que tal fórmula foi obtida por Darwin, G.,

$$
k_{2}(\sigma)=k_{f} \sqrt{\frac{1+\sigma^{2} \eta^{2} / \mu^{2}}{1+\sigma^{2} \eta^{2} / \mu^{2}(1+19 \mu / 2 g \bar{\rho} R)^{2}}},
$$

sendo $\sigma$ a frequência exercida por um potencial externo, $\bar{\rho}$ a densidade média do corpo e $k_{f}$ é o número de Love fluido, o valor que $k_{2}$ assumiria se o corpo fosse um fluido perfeito com a mesma 
distribuição de massa.

Vamos dar um sentido para esta afirmação no contexto do modelo apresentado. Já que é citada uma frequência de excitação (externa), considere que o satélite orbita o corpo em movimento circular com uma frequência $\sigma$. Analisemos a resposta do corpo neste regime forçado.

Tome $\vec{r}(t)=r_{0}(\cos (\tilde{\sigma} t)$, sen $(\tilde{\sigma} t), 0)$. Suponhamos que devido à alta inércia do corpo central, sua velocidade angular seja perpendicular ao plano deste movimento e não se altere. Desta forma,

$$
Y(t)=\left(\begin{array}{ccc}
\cos (\omega t) & -\operatorname{sen}(\omega t) & 0 \\
\operatorname{sen}(\omega t) & \cos (\omega t) & 0 \\
0 & 0 & 1
\end{array}\right), \quad \vec{r}^{T}=r_{0}^{2}\left(\begin{array}{ccc}
\cos ^{2}(\tilde{\sigma} t) & \operatorname{sen}(\tilde{\sigma} t) \cos (\tilde{\sigma} t) & 0 \\
\operatorname{sen}(\tilde{\sigma} t) \cos (\tilde{\sigma} t) & \operatorname{sen}^{2}(\tilde{\sigma} t) & 0 \\
0 & 0 & 0
\end{array}\right) .
$$

Novamente, usando a equação (3.57), e denotando por $\lambda_{1,2}$ as raízes de seu polinômio característico, obtemos

$$
\begin{gathered}
Y B Y^{T}=\left(\frac{1}{\lambda_{1} \lambda_{2}}+\frac{\lambda_{1} e^{\lambda_{1} t}-\lambda_{2} e^{\lambda_{2} t}}{\lambda_{1} \lambda_{2}\left(\lambda_{1}-\lambda_{2}\right)}\right) \operatorname{diag}\left(\frac{\omega^{2}}{3}+\frac{G m}{2 r_{0}^{3}}, \frac{\omega^{2}}{3}+\frac{G m}{2 r_{0}^{3}},-\frac{2 \omega^{2}}{3}-\frac{G m}{r_{0}^{3}}\right) \\
+\frac{3 G m}{2\left(\lambda_{1}-\lambda_{2}\right) r_{0}^{3}}\left(\begin{array}{ccc}
h_{1} & h_{2} & 0 \\
h_{2} & -h_{1} & 0 \\
0 & 0 & 0
\end{array}\right),
\end{gathered}
$$

onde,

$$
\begin{aligned}
& h_{1}=\frac{-\lambda_{1} \cos (2 \tilde{\sigma} t)+2 \sigma \operatorname{sen}(2 \tilde{\sigma} t)}{4 \sigma^{2}+\lambda_{1}^{2}}+\frac{\lambda_{2} \cos (2 \tilde{\sigma} t)-2 \sigma \operatorname{sen}(2 \tilde{\sigma} t)}{4 \sigma^{2}+\lambda_{2}^{2}}+\frac{\lambda_{1} e^{\lambda_{1} t}}{4 \sigma^{2}+\lambda_{1}^{2}}-\frac{\lambda_{2} e^{\lambda_{2} t}}{4 \sigma^{2}+\lambda_{2}^{2}}, \\
& h_{2}=-\frac{\lambda_{1} \operatorname{sen}(2 \tilde{\sigma} t)+2 \sigma \cos (2 \tilde{\sigma} t)}{4 \sigma^{2}+\lambda_{1}^{2}}+\frac{\lambda_{2} \operatorname{sen}(2 \tilde{\sigma} t)+2 \sigma \cos (2 \tilde{\sigma} t)}{4 \sigma^{2}+\lambda_{2}^{2}}+\frac{2 \sigma e^{\lambda_{1} t}}{4 \sigma^{2}+\lambda_{1}^{2}}-\frac{2 \sigma e^{\lambda_{2} t}}{4 \sigma^{2}+\lambda_{2}^{2}},
\end{aligned}
$$

sendo $\sigma:=\tilde{\sigma}-\omega$ a frequência do satélite no referencial do planeta.

Ao eliminarmos os termos transientes, obtemos

$$
\begin{aligned}
Y B Y^{T} & =\frac{1}{\lambda_{1} \lambda_{2}} \operatorname{diag}\left(\frac{\omega^{2}}{3}+\frac{G m}{2 r_{0}^{3}}, \frac{\omega^{2}}{3}+\frac{G m}{2 r_{0}^{3}},-\frac{2 \omega^{2}}{3}-\frac{G m}{r_{0}^{3}}\right) \\
& +\frac{3 G m}{2 r_{0}^{3}} k\left(\begin{array}{ccc}
\cos (2 \tilde{\sigma} t-\delta) & \operatorname{sen}(2 \tilde{\sigma} t-\delta) & 0 \\
\operatorname{sen}(2 \tilde{\sigma} t-\delta) & -\cos (2 \tilde{\sigma} t-\delta) & 0 \\
0 & 0 & 0
\end{array}\right),
\end{aligned}
$$

onde,

$$
\delta=\tan ^{-1}\left(\frac{2 \sigma \nu}{\tilde{\mu}-4 \sigma^{2}}\right), \quad k=\frac{1}{\sqrt{4 \sigma^{2} \nu^{2}+\left(\tilde{\mu}-4 \sigma^{2}\right)^{2}}} .
$$

Portanto, o movimento da matéria do planeta é dada pela seguinte expressão

$$
\begin{aligned}
Y(\operatorname{Id}+\varepsilon B)= & \tilde{\mu}^{-1}\left(\begin{array}{ccc}
a_{1}\left(\begin{array}{ccc}
\cos (\omega t) & -\operatorname{sen}(\omega t) \\
\operatorname{sen}(\omega t) & \cos (\omega t)
\end{array}\right) & 0 \\
0 & 0 & a_{2}
\end{array}\right) \\
& +\frac{3 G m k \varepsilon}{2 r_{0}^{3}}\left(\begin{array}{ccc}
\cos (\tilde{\sigma} t-\delta) & -\operatorname{sen}(\tilde{\sigma} t-\delta) & 0 \\
\operatorname{sen}(\tilde{\sigma} t-\delta) & \cos (\tilde{\sigma} t-\delta) & 0 \\
0 & 0 & 1
\end{array}\right)\left(\begin{array}{ccc}
\cos (\sigma t) & \operatorname{sen}(\sigma t) & 0 \\
\operatorname{sen}(\sigma t) & -\cos (\sigma t) & 0 \\
0 & 0 & 0
\end{array}\right),
\end{aligned}
$$

onde

$$
a_{1}=\tilde{\mu}+\varepsilon\left(\frac{\omega^{2}}{3}+\frac{G m}{2 r_{0}^{3}}\right), \quad a_{2}=\tilde{\mu}-\varepsilon\left(\frac{2 \omega^{2}}{3}-\frac{G m}{r_{0}^{3}}\right) .
$$

Neste caso, um cálculo simples mostra que $\lambda=\tilde{\mu}^{-1} a_{1}+3 G m k \varepsilon /\left(2 r_{0}^{3}\right)$ é o maior autovalor de 
Id $+\varepsilon B$, com autovetor $\vec{v}=(\cos (\tilde{\sigma} t-\delta / 2), \sin (\tilde{\sigma} t-\delta / 2), 0)$. Portanto, no estado estacionário a maré induzida pela interação segue a posição do corpo orbitante com um atraso de fase

$$
\Delta \varphi=\frac{1}{2} \tan ^{-1}\left(\frac{2 \sigma \nu}{\tilde{\mu}-4 \sigma^{2}}\right) .
$$

O comportamento desta solução estacionária é ilustrado na Figura 3.3. A solução assintótica do sistema completo [(3.58), (3.59),(3.60)] corresponde ao caso $\sigma=0$ e portanto $\Delta \varphi=0$.

A primeira matriz de (3.76) nos mostra que o planeta possui um achatamento permanente, resultante de sua rotação, sua elasticidade e da interação gravitacional (sobre si mesmo e até com o satélite). É o expresso pelas constantes $a_{1,2}$. Note que sua velocidade de rotação é a que atribuímos ao planeta, a maior parte da matéria do planeta se move com velocidade $\omega$, na forma de um elipsoide oblato.

A segunda matriz representa o movimento da maré, no plano do movimento do satélite com frequência $\tilde{\sigma}$ e com o atraso $\delta$.

Observação 3.22. Na expressão anterior, é simples verificar que ao trocar $\operatorname{Id}+B$ por $e^{B}$ a conclusão fica praticamente inalterada.

Continuando a análise, substitua esta expressão na equação (3.60) para obter:

$$
\begin{aligned}
\tilde{m} \ddot{\vec{r}} & =-\frac{G M m}{r^{3}} \vec{r}-\frac{3 G M m R^{2}}{r_{0}^{7}}\left(\frac{3}{5 \tilde{\mu}}\left(\frac{\omega^{2} r_{0}^{2}}{3}+\frac{G m}{2 r_{0}^{3}}\right)\right) \vec{r}+ \\
& -\frac{3 G m^{2} R^{5}}{r_{0}^{7}}\left(\frac{3 G M k}{R^{3}}\right)\left(\frac{3}{5} \frac{\vec{r}}{r_{0}}+\frac{\langle\vec{r}, \vec{f}\rangle}{r_{0}^{3}} \vec{r}-\frac{2}{5} \frac{\vec{f}}{r_{0}}\right),
\end{aligned}
$$

onde $\vec{f}(t)=\vec{r}_{a t}(t)-\vec{r}(t)$, sendo $\vec{r}_{a t}(t)=r_{0}(\cos (\sigma t-\delta)$, sen $(\sigma t-\delta))$, a posição do satélite num instante $t-\Delta t$, como indicado na Figura 3.3.

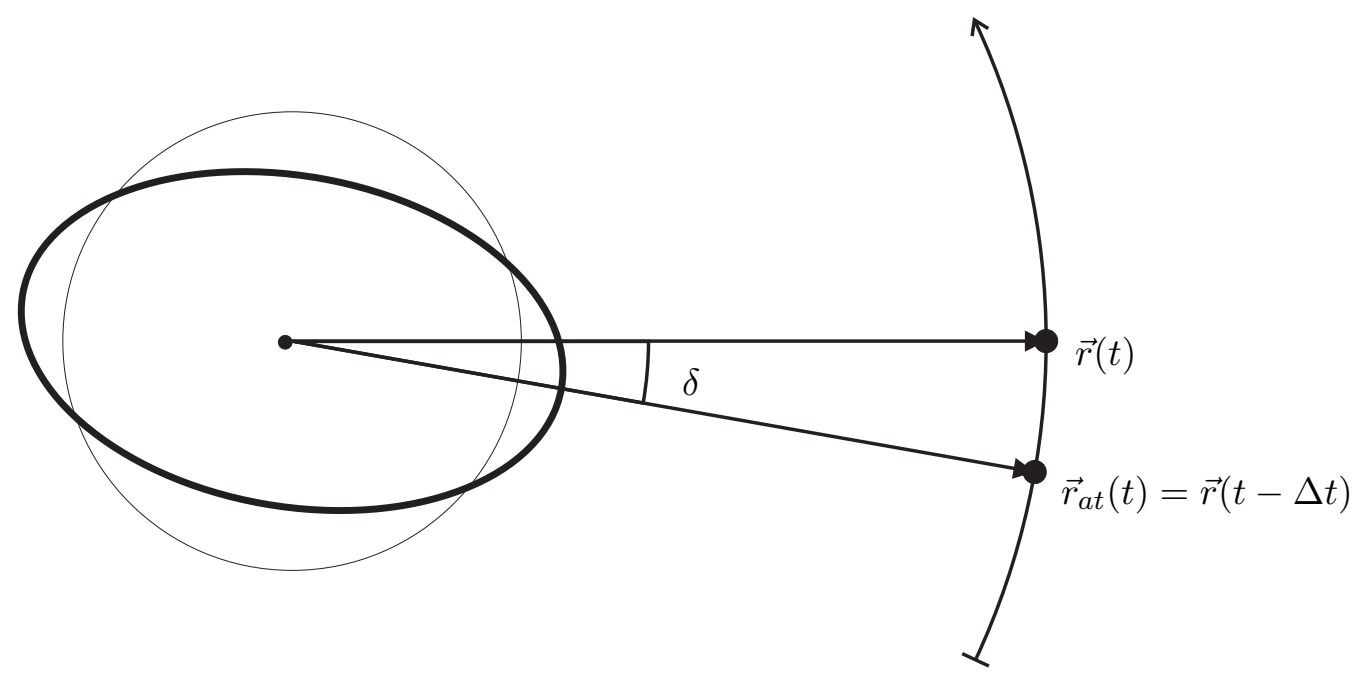

Figura 3.3: Atraso no surgimento da maré devido à viscosidade.

Agora, comparando com (3.63) ou diretamente com a equação (20) de [EL07], vemos que nosso modelo impõe:

$$
k_{2}(\sigma)=\frac{3 G M}{5 R^{3}} \frac{1}{\sqrt{4 \sigma^{2} \nu^{2}+\left(\tilde{\mu}-4 \sigma^{2}\right)^{2}}} .
$$

Observamos que há uma discrepância entre as expressões (3.73) e (3.78). Notamos que em [Dar08], página 18, a equação que origina o número de Love (3.73) é similar à equação (3.57), mas 
desprezando $\ddot{B}$. Neste caso, no regime forçado, desprezando a rotação do planeta, ela é equivalente a:

$$
\dot{B}+\frac{\tilde{\mu}}{\nu} B=\frac{G m}{2 \nu r_{0}^{5}}\left(\begin{array}{ccc}
3 \cos (2 \sigma t)+1 & 3 \operatorname{sen}(2 \sigma t) & 0 \\
3 \operatorname{sen}(2 \sigma t) & 1-3 \cos (2 \sigma t) & 0 \\
0 & 0 & -2
\end{array}\right) .
$$

Além disso, durante o cálculo é feita a troca da constante de viscosidade $1 / \eta$ pelo operador

$$
\left(\frac{1}{\tau}+\frac{d}{d t}\right)
$$

sendo a justificativa apresentada no texto ( $\tau$ é denominado módulo do tempo de relaxação da rigidez). Caso sigamos este método com (3.79), obteremos um número de Love próximo a (3.73) (com 19/2 trocado por 25/2).

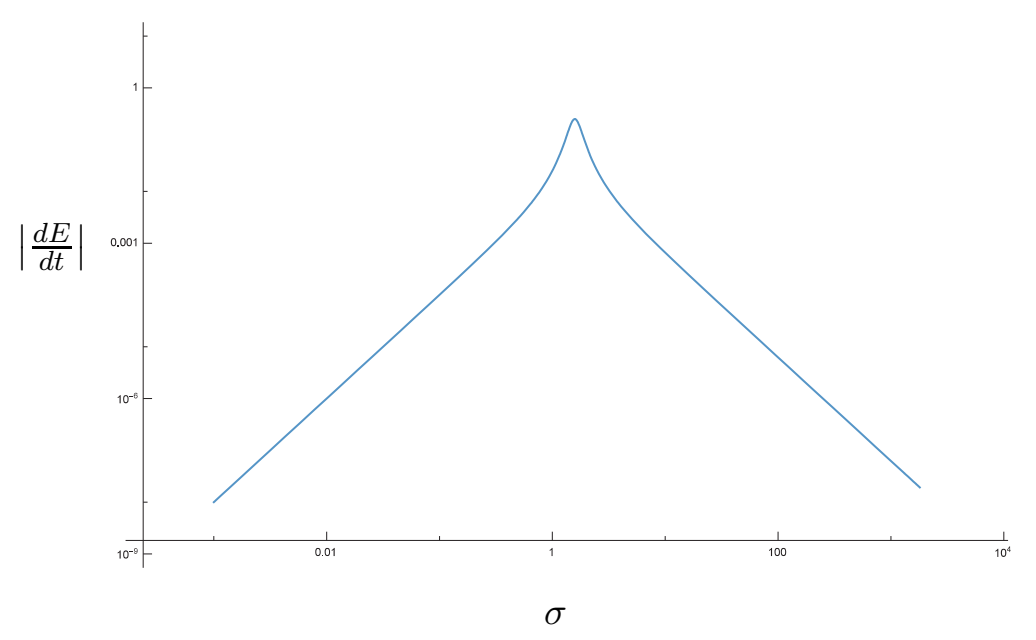

Figura 3.4: Potência dissipada em função da frequência.

Observamos que a forma da equação (3.79) se assemelha à equação de primeira ordem para a maré inelástica (creep equation) usada em [FM13], equação (5). Destacamos o fator $\gamma$ que ocorre naquela equação. Segundo sua definição,

$$
\gamma=\frac{3}{8} \frac{G M m}{\pi \eta R^{4}}
$$

sendo no texto informado que nos trabalhos de Darwin [Dar08] a mesma constante foi usada, mas com um fator $3 / 38$. No nosso modelo, a correspondente constante é, no caso homogêneo

$$
\gamma=\frac{\tilde{\mu}}{\nu}=\frac{3}{40} \frac{G M m}{\pi \eta R^{4}}
$$

mais próxima daquela usada por Darwin.

Resumindo esta discussão, o número de Love dependente de frequência e viscosidade é consequência do regime forçado do sistema, supondo que o satélite realiza um movimento periódico que não é alterado pela deformação do planeta (ou é muito lento em relação à oscilação). Isso esclarece porque não há esta dependência em (3.70), em que não fazemos tal restrição ao movimento.

Façamos agora o cálculo da potência dissipada, neste regime assintótico. Sabemos que, de (3.23),

$$
\frac{d E}{d t}=-2 \mathcal{D}=-\frac{8 \pi \eta R^{3}}{3} \operatorname{Tr}\left(\dot{B}^{2}\right)
$$


De (3.74), é fácil ver que

$$
B=D_{0}+\frac{3 G m}{2 r_{0}^{3}} k\left(\begin{array}{ccc}
\cos (2 \sigma t-\delta) & \operatorname{sen}(2 \sigma t-\delta) & 0 \\
\operatorname{sen}(2 \sigma t-\delta) & -\cos (2 \sigma t-\delta) & 0 \\
0 & 0 & 0
\end{array}\right)
$$

onde $D_{0}$ é matriz diagonal constante. Logo,

$$
\frac{d E}{d t}=-6\left(\frac{G m}{r_{0}^{3}}\right)^{2} \frac{\sigma^{2}}{4 \sigma^{2} \nu^{2}+\left(\tilde{\mu}-4 \sigma^{2}\right)^{2}} .
$$

Observamos que para $\sigma$ próximo de zero, $\dot{E} \approx-\alpha \sigma^{2}$. Para $\sigma$ grande, $\dot{E} \approx-\beta \sigma^{-2}$. Este é um comportamento parecido com aquele exibido em [FM13], apenas com uma lei de potência distinta. De fato, ao fazermos $\left(\tilde{\mu}-4 \sigma^{2}\right)^{2} \approx \tilde{\mu}^{2}$, obtemos uma expressão próxima àquela da energia total dissipada, obtida em [FM13]. Na Figura 3.4, temos o gráfico de $\dot{E}$ em função de $\sigma$, em unidades arbitrárias, e em escala Di-Log. Observamos que esta função aparenta ser simétrica em relação ao seu máximo, $\sigma=\sqrt{\tilde{\mu}} / 2$, apesar de não ser.

\subsubsection{Equilíbrios para o problema de dois corpos}

Como nos casos anteriores, o entendimento da dinâmica deste sistema passa pelo cálculo e propriedades da energia e do momento angular.

Seja a ação $\Phi: \mathrm{SO}(3) \times\left(\mathrm{SO}(3) \times \operatorname{SSym}_{+}(3) \times \mathbb{R}^{3}\right) \longrightarrow \mathrm{SO}(3) \times \operatorname{SSym}_{+}(3) \times \mathbb{R}^{3}, \Phi(R, Y, A, \vec{r}):=$ $(R Y, A, R \vec{r})$. Para todo $\xi \in \operatorname{skew}(3)$, verifica-se diretamente que $\mathcal{L}\left(d \Phi_{e^{s \xi}}((Y, A, \vec{r}),(\dot{Y}, \dot{A}, \dot{\vec{r}}))\right)=$ $\mathcal{L}\left(\left(e^{s \xi} Y, A, e^{s \xi} \vec{r}\right),\left(e^{s \xi} \dot{Y}, \dot{A}, e^{s \xi} \dot{\vec{r}}\right)\right)=\mathcal{L}((Y, A, \vec{r}),(\dot{Y}, \dot{A}, \dot{\vec{r}}))$. E como $\mathcal{D}$ não depende de $Y, \dot{Y}$, a segunda condição de (2.9) também é satisfeita. Portanto, de forma análoga aos casos anteriores, garantimos que o sistema admite a integral primeira:

$$
L=\frac{M R^{2} \varepsilon}{5} Y \Phi_{\varepsilon B}(\Omega) Y^{T}+\frac{\tilde{m}}{2} \varepsilon^{-\frac{2}{3}}\left(\dot{\vec{r}} \vec{r}^{T}-\vec{r} \dot{\vec{r}}^{T}\right),
$$

reconhecida como o momento angular total.

A energia, $E=T+V$, da mesma forma é tal que $\dot{E}=-2 \mathcal{D} \leq 0$, e $\dot{E} \equiv 0$ se e só se $B(t)$ é constante.

Sendo assim, para cada solução conservativa, $B(t)=B_{0}$, uma constante. De (3.57),

$$
B_{0}=\tilde{\mu}^{-1}\left(-\Omega^{2}+\frac{1}{3} \operatorname{Tr}\left(\Omega^{2}\right) \operatorname{Id}+\frac{3 G m}{r^{5}}\left(\left(Y^{T} \vec{r}\right)\left(Y^{T} \vec{r}\right)^{T}-\frac{1}{3} r^{2} \mathrm{Id}\right)\right) .
$$

Desta equação e de (3.59) decorre que $\Phi_{\varepsilon B}(\dot{\Omega})=0$. Assim, na região descrita pelo Lema 3.18, $\Omega(t)=\Omega_{0}$, uma constante.

Lema 3.23. Assuma que o corpo pseudo-rígido gira em torno do eixo $z, S\left(\Omega_{0}\right)=\left(0,0, \omega_{0}\right)$. Então o vetor posição relativa $\vec{r}$ descreve um movimento circular uniforme em torno deste eixo, com a mesma frequência $\omega_{0}$.

Demonstração. Podemos tomar

$$
Y(t)=\left(\begin{array}{ccc}
\cos \left(\omega_{0} t\right) & -\sin \left(\omega_{0} t\right) & 0 \\
\sin \left(\omega_{0} t\right) & \cos \left(\omega_{0} t\right) & 0 \\
0 & 0 & 1
\end{array}\right)
$$

Além disso, desde que $B$ e $\Omega$ são constantes, (3.84) implica que

$$
Z=\frac{1}{r^{5}} Y^{T}\left(\vec{r} \vec{r}^{T}-\frac{1}{3} r^{2} \mathrm{Id}\right) Y
$$


é constante. Note que $\|Z\|^{2}=2 /\left(3 r^{6}\right)$, portanto $r(t)=\|\vec{r}(t)\|$ também é constante. Considere as coordenadas $\vec{r}=\left(r_{1}, r_{2}, r_{3}\right)$ e note que a última componente de $Z$ é $r^{-5}\left(r_{3}^{2}-r^{2} / 3\right)$. Logo, $r_{3}(t)$ também é constante, assim como $r_{1}^{2}(t)+r_{2}^{2}(t)$. Então, podemos afirmar que $\vec{r}(t)=(a \cos (\alpha(t)), a \sin (\alpha(t)), c)$, onde $\alpha(t)$ é função suave e $a, c \in \mathbb{R}$ são constantes. Observe que a matriz constante $Z$ tem a forma

$$
Z=\frac{1}{3 r^{5}}\left(\begin{array}{ccc}
3 a^{2} \cos ^{2}(\varphi(t))-a^{2}-c^{2} & 3 a^{2} \cos (\varphi(t)) \sin (\varphi(t)) & 3 c a \cos (\varphi(t)) \\
3 a^{2} \cos (\varphi(t)) \sin (\varphi(t)) & 3 a^{2} \sin ^{2}(\varphi(t))-a^{2}-c^{2} & 3 c a \sin (\varphi(t)) \\
3 a c \cos (\varphi(t)) & 3 a c \sin (\varphi(t)) & 2 c^{2}-a^{2}
\end{array}\right)
$$

onde $\varphi(t)=\alpha(t)-\omega_{0} t$. Logo, $\alpha(t)=\omega_{0} t+\varphi_{0}$, e vemos que neste modelo as únicas soluções conservativas admitidas são as síncronas. Agora, defina $\tilde{\vec{r}}(t)=a\left(\cos \left(\omega_{0} t+\varphi_{0}\right), \sin \left(\omega_{0} t+\varphi_{0}\right), 0\right) \mathrm{e}$ observe que (3.60) se reduz a

$$
-\omega_{0}^{2} \tilde{\vec{r}}=\psi(\vec{r}) \vec{r}+\frac{6 G \tilde{M} \varepsilon^{\frac{8}{3}} \omega_{0}^{2} R^{2}}{5 r^{5}} \tilde{\vec{r}}
$$

onde $\psi(\vec{r})$ é uma função escalar. Note que, desde que $\tilde{\vec{r}} \neq \overrightarrow{0}$, (3.87) implica que $c=0$, ou seja, o plano de translação dos corpos é ortogonal ao eixo $z$.

Afirmamos que o maior semi-eixo deste elipsoide de equilíbrio está alinhado com o vetor posição relativa. De fato, defina $\vec{e}_{1}(t)=\left(\cos \left(\omega_{0} t+\varphi_{0}\right), \sin \left(\omega_{0} t+\varphi_{0}\right), 0\right), \vec{e}_{2}(t)=\left(-\sin \left(\omega_{0} t+\varphi_{0}\right), \cos \left(\omega_{0} t+\right.\right.$ $\left.\left.\varphi_{0}\right), 0\right)$ e $\vec{e}_{3}(t)=(0,0,1)$, e note que

$$
\begin{gathered}
Y\left(\mathrm{Id}+\varepsilon B_{0}\right) Y^{T} \vec{e}_{1}=\left(1+\frac{2}{3 \tilde{\mu}} \varepsilon \omega_{0}^{2}+\frac{2 G m \varepsilon}{\tilde{\mu} r^{3}}\right) \vec{e}_{1}, \\
Y\left(\mathrm{Id}+\varepsilon B_{0}\right) Y^{T} \vec{e}_{3}=\left(1-\frac{G m \varepsilon}{\tilde{\mu} r^{3}}\right) \vec{e}_{3}, \\
Y\left(\mathrm{Id}+\varepsilon B_{0}\right) Y^{T} \vec{e}_{2}=\left(1+\frac{2}{3 \tilde{\mu}} \varepsilon \omega_{0}^{2}-\frac{G m \varepsilon}{\tilde{\mu} r^{3}}\right) \vec{e}_{2},
\end{gathered}
$$

portanto suas direções correspondem aos eixos principais e o maior autovalor é associado a $\vec{e}_{1}=$ $r^{-1} \vec{r}$. Destacamos que não há atraso de fase neste estado final.

Agora, vejamos que (3.60) se torna equivalente a

$$
18 G^{2} \tilde{M} m R^{2} \tilde{\mu}^{-1} \varepsilon^{\frac{5}{3}}\left(r^{-1}\right)^{8}+6 G \tilde{M} R^{2} \tilde{\mu}^{-1} \varepsilon^{\frac{5}{3}} \omega_{0}^{2}\left(r^{-1}\right)^{5}+G \tilde{M}\left(r^{-1}\right)^{3}=\omega_{0}^{2},
$$

e observe que do lado esquerdo temos um polinômio em $r^{-1}$ cujos coeficientes são positivos, e portanto é monótono para $r>0$. Então, vemos que para cada $\omega_{0}^{2}(3.88)$ tem uma única raiz $r\left(\omega_{0}^{2}\right)$. Note que se $r \leq \beta:=\varepsilon^{\frac{1}{3}} \sqrt[5]{6 G \tilde{M} \tilde{\mu}^{-1} R^{2}}$, a equação (3.88) não possui solução, então o raio de todas órbitas circulares deste modelo devem ser maiores do que esta cota. Além disso, (3.88) é resolvida por uma função suave $\omega_{0}^{2}(r), \forall r>\beta$, a inversa da anterior, que é decrescente e tal que $\lim _{r \rightarrow \alpha+} \omega_{0}^{2}=+\infty, \lim _{r \rightarrow+\infty} \omega_{0}^{2}=0$ e $\lim _{r \rightarrow+\infty} r^{3} \omega_{0}^{2}=G \tilde{M}$. Logo, para cada $r>\beta$, a norma do momento angular (3.83) fica

$$
\|L\|(r)=\left(\frac{M R^{2} \varepsilon^{\frac{5}{3}}}{5}\left(1+\frac{2 \omega_{0}^{2}(r) \varepsilon}{3 \tilde{\mu}}+\frac{G m \varepsilon}{\tilde{\mu} r^{3}}\right)+\frac{\tilde{m} r^{2}}{2}\right) \omega_{0}(r) .
$$

Observe que $\lim _{r \rightarrow \beta+}\|L\|=\lim _{r \rightarrow+\infty}\|L\|=+\infty$. Então, vemos que $\|L\|$ atinge um mínimo global em algum $r_{0}>\beta$ (no pelo menos), além disso cada nível de momento angular maior admite no mínimo dois raios de equilíbrio. Note que este momento angular mínimo é não nulo. Destacamos que para $r$ próximo de $\beta$ a velocidade angular se torna ilimitada, o que viola a condição $\left\|B_{0}\right\|<(2 \varepsilon)^{-1}$. Assim, é razoável que o raio externo corresponda ao único equilíbrio estável.

Ressaltamos o fato de que nenhuma condição inicial cuja norma de seu momento angular seja 
menor do que o mínimo de (3.89) gera uma solução limitada e que satisfaça $\|B(t)\|<1 /(2 \varepsilon)$, para todo $t \geq 0$. Além disso, lembramos que a existência de um raio mínimo para as soluções de equilíbrio não é tão surpreendente desde que na literatura temos o conhecido raio de Roche, veja Capítulo 8 de [Cha87] por exemplo. No entanto, note que os métodos que usamos para determinar tal raio mínimo são distintos dos usados nesta referência.

Substituindo os valores que $\tilde{\mu}$ assume para um corpo homogêneo e revertendo a escala nas coordenadas, obtemos a expressão para o raio mínimo

$$
r_{\text {min }}=\left(\frac{15(M+m)}{2 M} \frac{1}{\left(1+\frac{25}{2} \frac{\mu}{g \rho R}\right)}\right)^{\frac{1}{5}} R .
$$

Desprezando as razões $\mu /(g \rho R)$ e $m / M$, obtemos $r_{\text {min }} \approx 1.2394 \cdot R$.

Por fim, desprezando os efeitos da rotação $\Omega$, observe como fica a matriz $B_{0}$ no equilíbrio (eliminando a escala $\varepsilon$ ):

$$
B_{0}=\frac{G m}{\tilde{\mu} r^{3}}\left(\begin{array}{ccc}
2 & 0 & 0 \\
0 & -1 & 0 \\
0 & 0 & -1
\end{array}\right) .
$$

Notamos que se forma um equilíbrio do tipo elipsoide de revolução sendo o eixo de simetria a reta que liga os centros de massa dos corpos. A eliticidade da elipse dada pela interseção com o plano de rotação então fica:

$$
\epsilon=e^{b_{2}}-e^{b_{1}} \approx b_{2}-b_{1}=-\frac{3 G m}{\tilde{\mu} r^{3}}=-\frac{15}{4} \frac{m R^{3}}{M r^{3}\left(1+\frac{25}{2} \frac{\mu}{g \rho R}\right)} \approx-\frac{15}{4} \frac{m}{M}\left(\frac{R}{r}\right)^{3},
$$

sendo que na última equação fizemos o módulo $\mu=0$. Compare com a equação (1.9).

Observamos que o Princípio de LaSalle não é facilmente aplicado neste caso, pois os níveis de energia deixam de ser compactos, sendo mais complicado dar condições para que os corpos não se colidam. Também destacamos a concordância dos resultados obtidos nesta seção com aqueles apresentados em [BH12], sendo que neste trabalho os autores deduzem várias das conclusões obtidas aqui fazendo hipóteses básicas sobre um sistema menos concreto. Ressaltamos que nosso modelo satisfaz a todas as hipóteses listadas no artigo.

\subsection{Determinação da constante efetiva $\tilde{\mu}$}

Como foi dito neste capítulo, não é essencial a hipótese de que a densidade de massa do corpo seja homogênea. Aliás, mesmo o módulo e a viscosidade de cisalhamento $\mu$ e $\eta$ podem ser adotados como radialmente simétricos. O fato é que as constantes $\tilde{\mu}$ e $\nu$ que ocorrem em (3.24) podem ser consideradas como efetivas, ou seja, não serão obtidas através das informações internas (moleculares) dos corpos, mas indiretamente através de medidas.

Trataremos apenas o caso da constante $\tilde{\mu}$. Lembremos a definição do tensor de quadrupolo de uma distribuição de massa $\rho$

$$
Q=\int_{\mathcal{B}}\left(3 \phi(x) \phi(x)^{T}-\|\phi(x)\|^{2} \mathrm{Id}\right) \rho(\|x\|, t) d x .
$$

Impondo que $\phi(x)=A x$, temos

$$
Q=\int_{\mathcal{B}}\left(3 A x x^{T} A-\|A x\|^{2}\right) \rho(\|x\|, t) d x .
$$


Tabela 3.2: Dados de achatamento dos corpos do Sistema Solar

\begin{tabular}{llllll}
\hline Corpo & $\omega\left(10^{-5} s^{-1}\right)$ & $I_{\circ} / M R^{2}$ & $J_{2}\left(10^{-6}\right)$ & $\tilde{\mu}\left(10^{-6} s^{-2}\right)$ & $\tilde{\gamma}$ \\
\hline Sol & 0,338 & 0,059 & 0,218 & 3,092 & 0,5562 \\
Mercúrio & 0,1240 & 0,35 & 50,3 & 0,0107 & 0,0072 \\
Vênus & 0,0299 & 0,33 & 4,458 & 0,0066 & 0,0042 \\
Terra & 7,2921 & 0,3308 & 1082,63 & 1,625 & 0,9909 \\
Lua & 0,26617 & 0,394 & 202,7 & 0,01377 & 0,0180 \\
Marte & 7,0882 & 0,366 & 1960,45 & 0,9380 & 0,9331 \\
Júpiter & 17,5852 & 0,254 & 14736, & 0,5330 & 0,9093 \\
Saturno & 16,3788 & 0,21 & 16298, & 0,3457 & 0,8556 \\
Urano & 10,1237 & 0,225 & 3343,43 & 0,6897 & 1,024 \\
Netuno & 10,8338 & 0,2555 & 3411, & 0,8792 & 1,225 \\
\hline
\end{tabular}

Portanto, usando resultados anteriores e a identidade

$$
\int_{\mathcal{B}} x x^{T} \rho(\|x\|, t) d x=\frac{\mathrm{I}_{\circ}}{2} \mathrm{Id},
$$

concluímos que

$$
Q=\frac{\mathrm{I}_{\circ}}{2}\left(3 A^{2}-\operatorname{Tr}\left(A^{2}\right) \mathrm{Id}\right) \approx 3 \mathrm{I}_{\circ} B,
$$

onde tomamos uma aproximação em primeira ordem em $B$. Vemos assim que $B$ é apenas um múltiplo do tensor de quadrupolo, $B=Q /\left(3 \mathrm{I}_{\circ}\right)$.

Supondo rotação ao redor do eixo $z, S(\Omega)=(0,0, \omega)$, e deformação

$$
Q=3 \mathrm{I}_{\circ} B=\left(\begin{array}{ccc}
\lambda & 0 & 0 \\
0 & \lambda & 0 \\
0 & 0 & -2 \lambda
\end{array}\right),
$$

introduzimos o fator dinâmico $J_{2}=\lambda / M R^{2}$. Seus valores medidos no Sistema Solar estão na quarta coluna da Tabela 3.2. Assim, a equação (3.24) implica que

$$
\tilde{\mu}=\frac{\mathrm{I}_{\circ}}{M R^{2}} \frac{\omega^{2}}{J_{2}} .
$$

Além disso, podemos observar da Tabela 3.2 que a hipótese de homogeneidade da massa é drástica em vários casos. O momento de inércia de uma esfera homogênea é $I=0,4 M R^{2}$, mas constatamos da terceira coluna que esta raramente é uma boa aproximação. Desta forma, considere o raio efetivo do corpo como sendo $R_{g}=\sqrt{5 \mathrm{I}_{\circ} / 2 M}$, o raio de um corpo homogêneo que possui o mesmo momento de inércia medido. Para este corpo, desprezando a parte elástica, a constante obtida em (3.25) seria

$$
\tilde{\mu}=\frac{4 G M}{5 R_{g}^{3}} .
$$

Na última coluna da Tabela 3.2 comparamos estes dois valores,

$$
\tilde{\gamma}:=\frac{\tilde{\mu}}{\frac{4 G M}{5 R_{g}^{3}}}
$$

onde $\tilde{\mu}$ vem da equação (3.93). 
Notamos que os maiores desvios foram observados em Mercúrio, Vênus, Lua e no Sol. No caso deste último, o desvio pode ser causado pela falta de simetria radial em sua distribuição de massa, ou por velocidades de rotação distintas em camadas internas. No caso dos corpos menores, uma possível explicação para o desvio seria porque não possuem formato esférico quando em repouso, especialmente a Lua. Ou seja, possuiriam uma deformação permanente, violando uma de nossas hipóteses.

A maioria dos dados da Tabela 3.2 também foram retirados de (http://nssdc.gsfc.nasa.gov/ planetary/planetfact.html). O valor $J_{2}=2,18 \times 10^{-7}$ do Sol foi tirado de [ACG08], ver também [RD11]. O momento de inércia de Netuno $\left(\mathrm{I}_{\circ} /\left(M R^{2}\right)=0,2555\right)$ foi tirado de [NHFR13].

\subsection{Corpo Assimétrico e Ressonância Spin-Órbita}

Em todas as discussões expostas neste capítulo, supusemos que o corpo pseudo-rígido em repouso assume o formato esférico. Matematicamente, tal informação está contida na definição da energia potencial elástica (2.26), quando escolhemos $\mathcal{B} \subset \mathbb{R}^{3}$. Nesta seção, suporemos que no equilíbrio o corpo seja um elipsoide quase esférico, $\mathcal{B}=A_{0}\left(B_{R}(0)\right)$, onde $A_{0}=e^{\varepsilon D_{0}}, D_{0}=\operatorname{diag}\left(d_{1}, d_{2},-d_{1}-d_{2}\right)$, $0<\varepsilon<<1$.

Com este mesmo parâmetro, usaremos as escalas relativas discutidas no início da Seção 3.4. A energia cinética $T_{1}(3.55)$ fica

$$
T_{1}=\frac{\rho}{2} \int_{B_{R}(0)}\left\|\sqrt{\varepsilon} \Omega A A_{0} x+\dot{A} A_{0} x+Y^{T} \dot{\vec{P}}\right\|^{2} d x
$$

O potencial gravitacional do corpo pseudo-rígido (3.6) fica

$$
V_{s g}(A)=-\frac{\rho^{2} G}{2} \int_{B_{R}(0)} \int_{B_{R}(0)} \frac{1}{\left\|A A_{0}(x-y)\right\|} d x d y .
$$

O potencial de interação fica

$$
V_{\text {int }}=-G m \rho \varepsilon^{\frac{1}{3}} \int_{B_{R}(0)} \frac{1}{\left\|Y^{T} \vec{r}-\varepsilon^{\frac{1}{3}} A A_{0} x\right\|} d x .
$$

Observe que o potencial elástico e a função dissipação não se alteram neste novo contexto. Note também que $A A_{0}=e^{\varepsilon B} e^{\varepsilon D_{0}}=\mathrm{Id}+\varepsilon\left(B+D_{0}\right)+\varepsilon^{2}\left(B^{2}+2 B D_{0}+D_{0}^{2}\right) / 2+\mathcal{O}\left(\varepsilon^{3}\right)$. Assim, usando argumentos similares aos apresentados no Apêndice B, vemos que $d V_{s g} /\left.d \varepsilon\right|_{\varepsilon=0}=0$, e como em (B.1),

$$
\begin{array}{r}
\left.\frac{d^{2}}{d \varepsilon^{2}}\right|_{\varepsilon=0} V_{s g}=G \rho^{2}\left(\int_{\mathcal{B}} \int_{\mathcal{B}} \frac{\left\|\left(B+D_{0}\right)(x-y)\right\|^{2}}{\|x-y\|^{3}}+\frac{\left\langle x-y,\left(B^{2}+2 B D_{0}+D_{0}^{2}\right)(x-y)\right\rangle}{\|x-y\|^{3}}\right. \\
\left.-3 \frac{\left\langle x-y,\left(B+D_{0}\right)(x-y)\right\rangle^{2}}{\|x-y\|^{5}} d x d y\right) .
\end{array}
$$

Desde que $B$ e $D_{0}$ são simétricas, $\left\langle x-y, 2 B D_{0}(x-y)\right\rangle=\left\langle x-y,\left(B D_{0}+D_{0} B\right)(x-y)\right\rangle$. Logo,

$$
\left.\frac{d^{2}}{d \varepsilon^{2}}\right|_{\varepsilon=0} V_{s g}=G \rho^{2}\left(\int_{\mathcal{B}} \int_{\mathcal{B}} 2 \frac{\left\|\left(B+D_{0}\right)(x-y)\right\|^{2}}{\|x-y\|^{3}}-3 \frac{\left\langle x-y,\left(B+D_{0}\right)(x-y)\right\rangle^{2}}{\|x-y\|^{5}} d x d y\right) .
$$

Assim, de forma análoga à equação (B.6), temos

$$
V_{s g}=\frac{2}{25} \frac{G M^{2}}{R} \varepsilon^{2} \operatorname{Tr}\left(\left(B+D_{0}\right)^{2}\right)+\mathcal{O}\left(\varepsilon^{3}\right) .
$$


Portanto, a Lagrangiana deste problema é

$$
\begin{gathered}
\varepsilon^{\frac{2}{3}} \mathcal{L}=\frac{M R^{2}}{10}\left(\varepsilon^{\frac{8}{3}} \operatorname{Tr}\left(\dot{B}^{2}\right)-\varepsilon^{\frac{5}{3}} \operatorname{Tr}\left(\Omega^{2}\right)-2 \varepsilon^{\frac{8}{3}} \operatorname{Tr}\left(\Omega^{2}\left(B+D_{0}\right)\right)-\frac{4 G M}{5 R^{3}} \varepsilon^{\frac{8}{3}} \operatorname{Tr}\left(\left(B+D_{0}\right)^{2}\right)\right. \\
\left.-\frac{10 \mu}{\rho R^{2}} \varepsilon^{\frac{8}{3}} \operatorname{Tr}\left(B^{2}\right)\right)+\frac{1}{2} \tilde{m} \dot{\vec{r}}^{2}+\frac{\tilde{M}}{2} \dot{\vec{R}}_{C M}^{2}+\frac{G m M}{\|\vec{r}\|} \varepsilon+\frac{3 G m M R^{2}}{5 r^{5}} \varepsilon^{\frac{8}{3}} \operatorname{Tr}\left(\left(Y^{T} \vec{r}\right)\left(Y^{T} \vec{r}\right)^{T}\left(B+D_{0}\right)\right)+\mathcal{O}\left(\varepsilon^{\frac{19}{6}}\right) .
\end{gathered}
$$

Observamos que não há diferença essencial no cálculo das equações de movimento,

$$
\begin{gathered}
\ddot{B}+\nu \dot{B}+\tilde{\mu} B=-\frac{4 G M}{5 R^{3}} D_{0}-\Omega^{2}+\frac{1}{3} \operatorname{Tr}\left(\Omega^{2}\right) \operatorname{Id}+\frac{3 G m}{r^{5}}\left(\left(Y^{T} \vec{r}\right)\left(Y^{T} \vec{r}\right)^{T}-\frac{1}{3} r^{2} \mathrm{Id}\right) \\
\dot{\Omega}+\varepsilon\left(\left(B+D_{0}\right) \dot{\Omega}+\dot{\Omega}\left(B+D_{0}\right)+\Omega \dot{B}+\dot{B} \Omega\right)=\varepsilon\left[B+D_{0}, \Omega^{2}\right]+\frac{3 G m}{r^{5}} \varepsilon\left[\left(Y^{T} \vec{r}\right)\left(Y^{T} \vec{r}\right)^{T}, B+D_{0}\right] \\
\ddot{\vec{r}}=-\frac{G \tilde{M} \varepsilon}{r^{3}} \vec{r}-\frac{3 G \tilde{M} \varepsilon^{\frac{8}{3}} R^{2}}{r^{7}}\left\langle Y\left(B+D_{0}\right) Y^{T} \vec{r}, \vec{r}\right\rangle \vec{r}+\frac{6 G \tilde{M} \varepsilon^{\frac{8}{3}} R^{2}}{5 r^{5}} Y\left(B+D_{0}\right) Y^{T} \vec{r} .
\end{gathered}
$$

Como primeira implicação destas equações, podemos repetir a análise da obtenção do sistema (3.71). Notamos que todos os termos que ocorrem naquela equação permanecem inalterados. Do lado direito da igualdade, surgem os três novos termos

$$
\frac{10 \mu}{\rho R^{2} \tilde{\mu}} \varepsilon\left(-\left(D_{0} \tilde{\Omega}^{\prime}+\tilde{\Omega}^{\prime} D_{0}\right)+\left[D_{0}, \tilde{\Omega}^{2}\right]+\frac{3 G m}{r^{5}}\left[\left(Y^{T} \vec{r}\right)\left(Y^{T} \vec{r}\right)^{T}, D_{0}\right]\right) .
$$

Impondo as mesmas restrições para a órbita circular, notamos que $\left[D_{0}, \tilde{\Omega}^{2}\right]=0, D_{0} \tilde{\Omega}^{\prime}+\tilde{\Omega}^{\prime} D_{0}=$ $\left(d_{1}+d_{2}\right) \tilde{\Omega}^{\prime}, \mathrm{e}$

$$
\left[\left(Y^{T} \vec{r}\right)\left(Y^{T} \vec{r}\right)^{T}, D_{0}\right]=\frac{d_{1}-d_{2}}{2} \operatorname{sen}(2(\omega-\sigma) t) r_{0}^{2}\left(\begin{array}{cc}
0 & -1 \\
1 & 0
\end{array}\right) .
$$

Portanto, obtemos uma equação escalar, extensão de (3.72)

$$
I_{\varepsilon}(\omega) \omega^{\prime}(t)=-\frac{9 G^{2} m^{2} \nu \sqrt{\varepsilon}}{\tilde{\mu}^{2} r_{0}^{6}}(\omega-\sigma)+\frac{15 G m \mu}{\rho R^{2} \tilde{\mu} r_{0}^{3}} \varepsilon\left(d_{1}-d_{2}\right) \operatorname{sen}(2(\omega(t)-\sigma t)),
$$

onde

$$
I_{\varepsilon}(\omega)=\left(1+\frac{\varepsilon}{\tilde{\mu}}\left(\frac{2 \omega^{2}}{3}+\frac{G m}{r_{0}^{3}}+\frac{10 \mu}{\rho R^{2}}\left(d_{1}+d_{2}\right)\right)\right)
$$

faz o papel de um tensor de inércia. Observe que tomando $I_{\varepsilon}(\omega)=1$, temos a equação (1) de [ABC14], restrita a um movimento circular. A equação (3.102) é usada no estudo de captura em ressonância spin-órbita, por exemplo no caso de Mercúrio com o Sol. Disto, podemos esperar que em nosso modelo completo também haja tais capturas, até mesmo sem a necessidade de supor uma deformação permanente, como é o caso do modelo estudado em [CBLR14].

\subsection{Problema de N Corpos}

Considere a extensão natural do problema de dois corpos da Seção 3.4, qual seja, N corpos pseudo-rígidos interagindo gravitacionalmente cujo formato de repouso é esférico. Trataremos o caso de pequenas deformações, com a mesma escala utilizada na referida seção. Observamos que as energias cinética, potencial elástica, potencial auto-gravitacional e a função dissipação do sistema completo são aditivas, ou seja, as obtemos usando as mesmas fórmulas anteriores para cada corpo e as somando em seguida. A única dificuldade surge no potencial de interação.

Considere dois corpos pseudo-rígidos homogêneos cujo centro de massa tem vetor posição $\vec{P}_{i}$, 
$\vec{P}_{j}$. Sendo $\vec{r}_{i j}=\vec{P}_{i}-\vec{P}_{j}$, a energia de interação é

$$
V_{i j}^{i n t}(\varepsilon)=-G \rho_{i} \rho_{j} \int_{\mathcal{B}_{j}} \int_{\mathcal{B}_{i}} \frac{1}{\left\|Y_{i} e^{\varepsilon B_{i}} x-Y_{j} e^{\varepsilon B_{j}} y+\vec{r}_{i j}\right\|} d x d y .
$$

Como os domínios de integração são bolas, vemos que

$$
\begin{gathered}
V_{i j}^{i n t}(0)=-G \rho_{i} \rho_{j} \int_{\mathcal{B}_{j}} \int_{\mathcal{B}_{i}} \frac{1}{\left\|x-y+\vec{r}_{i j}\right\|} d x d y=-\frac{G M_{i} M_{j}}{\left\|\vec{r}_{i j}\right\|}, \\
\left.\frac{d}{d \varepsilon}\right|_{\varepsilon=0} V_{i j}^{i n t}(\varepsilon)=G \rho_{i} \rho_{j} \int_{\mathcal{B}_{j}} \int_{\mathcal{B}_{i}} \frac{\left\langle x-y+\vec{r}_{i j}, Y_{i} B_{i} Y_{i}^{T} x-Y_{j} B_{j} Y_{j}^{T} y\right\rangle}{\left\|x-y+\vec{r}_{i j}\right\|^{3}} d x d y .
\end{gathered}
$$

Notando a simetria desta expressão em relação aos índices $i, j$, basta calcular

$$
\int_{\mathcal{B}_{j}} \int_{\mathcal{B}_{i}} \frac{\left\langle x-y+\vec{r}_{i j}, Y_{j} B_{j} Y_{j}^{T} y\right\rangle}{\left\|x-y+\vec{r}_{i j}\right\|^{3}} d x d y=\int_{\mathcal{B}_{j}}\left\langle Y_{j} B_{j} Y_{j} y, \int_{\mathcal{B}_{i}} \frac{y-\vec{r}_{i j}-x}{\left\|y-\vec{r}_{i j}-x\right\|^{3}} d x\right\rangle d y .
$$

Lembrando que

$$
\int_{\mathcal{B}_{i}} \frac{y-\vec{r}_{i j}-x}{\left\|y-\vec{r}_{i j}-x\right\|^{3}} d x=\frac{4 \pi R_{i}^{3}}{3} \frac{y-\vec{r}_{i j}}{\left\|y-\vec{r}_{i j}\right\|^{3}}
$$

a expressão (3.103) fica

$$
\frac{4 \pi R_{i}^{3}}{3} \int_{\mathcal{B}_{j}} \frac{\left\langle Y_{j} B_{j} Y_{j} y, y-\vec{r}_{i j}\right\rangle}{\left\|y-\vec{r}_{i j}\right\|^{3}} d x d y=\frac{4 \pi R_{i}^{3}}{3} \frac{4 \pi R_{j}^{5}}{5\left\|\vec{r}_{i j}\right\|^{5}} \operatorname{Tr}\left(\left(Y_{j}^{T} \vec{r}_{i j}\right)\left(Y_{j}^{T} \vec{r}_{i j}\right)^{T} B_{j}\right),
$$

sendo que na última passagem foi usada novamente a equação (3.103). Portanto,

$$
\left.\frac{d}{d \varepsilon}\right|_{\varepsilon=0} V_{i j}^{i n t}(\varepsilon)=-\frac{3 G M_{i} M_{j}}{\left\|\vec{r}_{i j}\right\|^{5}} \operatorname{Tr}\left(\vec{r}_{i j} \vec{r}_{i j}^{T}\left(R_{i}^{2} Y_{i} B_{i} Y_{i}^{T}+R_{j}^{2} Y_{j} B_{j} Y_{j}^{T}\right) .\right.
$$

Usando novamente a escala $\vec{r}_{i j} \rightarrow \varepsilon^{-\frac{1}{3}} \vec{r}_{i j}$, temos

$$
V_{i j}^{i n t}=-\frac{G M_{i} M_{j} \varepsilon^{\frac{1}{3}}}{\left\|\vec{r}_{i j}\right\|}-\frac{3 G M_{i} M_{j} \varepsilon^{2}}{\left\|\vec{r}_{i j}\right\|^{5}} \operatorname{Tr}\left(\vec{r}_{i j} \vec{r}_{i j}^{T}\left(R_{i}^{2} Y_{i} B_{i} Y_{i}^{T}+R_{j}^{2} Y_{j} B_{j} Y_{j}^{T}\right)+\mathcal{O}\left(\varepsilon^{3}\right) .\right.
$$

Assim, a Lagrangiana e a função dissipação ficam

$$
\mathcal{L}=\sum_{i=1}^{N}\left(T_{i}-\tilde{\mu}_{i} \varepsilon^{2} \operatorname{Tr}\left(B_{i}^{2}\right)\right)-\sum_{i<j} V_{i j}^{i n t}, \quad \mathcal{D}=\sum_{i=1}^{N} \varepsilon^{2} \frac{4 \pi \eta_{i} R_{i}^{3}}{3} \operatorname{Tr}\left(\dot{B}_{i}{ }^{2}\right)+\mathcal{O}\left(\varepsilon^{3}\right) .
$$

As equações de movimento agora são, para cada $i=1, \cdots, N$,

$$
\begin{gathered}
\ddot{B}_{i}+\nu_{i} \dot{B}_{i}+\tilde{\mu}_{i} B_{i}=-\Omega_{i}^{2}+\frac{1}{3} \operatorname{Tr}\left(\Omega_{i}^{2}\right) \mathrm{Id}+\sum_{j \neq i} \frac{3 G M_{j}}{r_{i j}^{5}}\left(\left(Y_{i}^{T} \vec{r}_{i j}\right)\left(Y_{i}^{T} \vec{r}_{i j}\right)^{T}-\frac{1}{3} r_{i j}^{2} \mathrm{Id}\right) \\
\dot{\Omega}_{i}+\varepsilon\left(B_{i} \dot{\Omega}_{i}+\dot{\Omega}_{i} B_{i}+\Omega_{i} \dot{B}_{i}+\dot{B}_{i} \Omega_{i}\right)=\varepsilon\left[B_{i}, \Omega_{i}^{2}\right]+\sum_{j \neq i} \frac{3 G M_{j}}{r_{i j}^{5}} \varepsilon\left[\left(Y_{i}^{T} \vec{r}_{i j}\right)\left(Y_{i}^{T} \vec{r}_{i j}\right)^{T}, B_{i}\right]
\end{gathered}
$$




$$
\begin{aligned}
\ddot{\vec{P}}_{i}=\sum_{j \neq i}-\frac{G M_{j} \varepsilon}{r_{i j}^{3}} \vec{r}_{i j}-\frac{3 G M_{j} \varepsilon^{\frac{8}{3}}}{r_{i j}^{7}}\left\langle\left( R_{i}^{2} Y_{i} B_{i} Y_{i}^{T}\right.\right. & \left.\left.+R_{j}^{2} Y_{j} B_{j} Y_{j}^{T}\right) \vec{r}_{i j}, \vec{r}_{i j}\right\rangle \vec{r}_{i j} \\
& +\sum_{j \neq i} \frac{6 G M_{j} \varepsilon^{\frac{8}{3}}}{5 r_{i j}^{5}}\left(R_{i}^{2} Y_{i} B_{i} Y_{i}^{T}+R_{j}^{2} Y_{j} B_{j} Y_{j}^{T}\right) \vec{r}_{i j} .
\end{aligned}
$$

Para dois corpos pseudo-rígidos, os equilíbrios relativos deste sistemas são bem entendidos. A prova foi praticamente desenvolvida na Seção 3.4.2.

Proposição 3.24. Em qualquer solução $\omega$-limite, portanto conservativa, do problema de dois corpos, ambos realizam um movimento circular uniforme em torno do centro de massa, suas velocidades angulares são constantes e perpendiculares ao plano da órbita, além disso seus movimentos de rotação e translação são síncronos.

Demonstração. Como $\mathcal{D}$ ainda é função homogênea quadrática das velocidades, $\dot{E}=-\mathcal{D} \leq 0$, sendo $E=T+V$. Para uma solução periódica, $\mathcal{D} \equiv 0$. Portanto, temos que $\dot{B}_{1}(t)=\dot{B}_{2}(t)=0, \forall t>0$. Das equações (3.105) e (3.106) vemos que

$$
\varepsilon\left[B_{i}, \Omega_{i}^{2}\right]+\frac{3 G M_{j}}{r_{12}^{5}} \varepsilon\left[\left(Y_{i}^{T} \vec{r}_{12}\right)\left(Y_{i}^{T} \vec{r}_{12}\right)^{T}, B_{i}\right]=0, \quad i, j=1,2,
$$

$\operatorname{logo}, \dot{\Omega}_{1}(t)=\dot{\Omega}_{2}(t)=0$, no domínio do sistema.

Portanto, novamente de (3.105), vemos que as matrizes

$$
Z_{i}=\frac{1}{r_{12}^{5}} Y_{i}^{T}\left(\vec{r}_{12} \vec{r}_{12}^{T}-\frac{1}{3} r_{12}^{2} \mathrm{Id}\right) Y_{i}, \quad i=1,2,
$$

também são constantes. Note que esta é a expressão (3.86). Concluímos que $\vec{r}_{12}$ também descreve um movimento circular uniforme em um plano ortogonal a $S\left(\Omega_{1}\right)=S\left(\Omega_{2}\right)$, com frequência $\omega=$ $\left\|\Omega_{1}\right\|=\left\|\Omega_{2}\right\|$.

Para $N>2$, é possível que ocorra movimento relativo entre os corpos sem que haja dissipação de energia. Acredita-se que tais situações não sejam possíveis caso a deformação seja não linear. Em um modelo mais completo, a deformação de um corpo seria constante somente se a posição dos demais em relação a este fosse fixa.

Considere novamente o problema de $\mathrm{N}$ corpos pseudo-rígidos. O mesmo raciocínio usado na Proposição 3.24 nos garante que toda solução periódica do sistema é tal que $B_{i}(t)$ e $\Omega_{i}(t)$ são matrizes constantes, $i=1, \cdots, N$. Mediante a observação do último parágrafo, faremos a hipótese de que a maré induzida pelo corpo de massa $M_{j}$ sobre o corpo de massa $M_{i}$,

$$
\frac{3 G M_{j}}{r_{i j}^{5}}\left(\left(Y_{i}^{T} \vec{r}_{i j}\right)\left(Y_{i}^{T} \vec{r}_{i j}\right)^{T}-\frac{1}{3} r_{i j}^{2} \mathrm{Id}\right)
$$

é constante, para todos $i, j=1, \cdots, N, i \neq j$.

Assim, usando o mesmo argumento da Proposição 3.24 e por transitividade, concluímos que todas as velocidades angulares $\Omega_{i}$ são iguais, os centros de massa de todos os corpos devem permanecer no mesmo plano, ortogonal a todas $S\left(\Omega_{i}\right)$, com distância mútua constante, descrevendo um movimento circular uniforme em torno do centro de massa, com velocidade angular $\omega=\left\|\Omega_{i}\right\|$. A situação é ilustrada na Figura 3.5.

Ressaltamos a dificuldade em determinar a posição de cada corpo em uma destas configurações de equilíbrio. Estas posições são soluções da equação (3.107) após substituirmos $Y_{k} B_{k} Y_{k}^{T}$, usando (3.105). Ao menos, nesta situação, o campo de forças resultante se torna central, portanto o momento angular orbital passa a ser conservado. De fato, o Apêndice D garante que (3.107) fica na forma (D.1). Observe que esta equação é uma perturbação das equações de configurações centrais, bem conhecidas em Mecânica Celeste. 


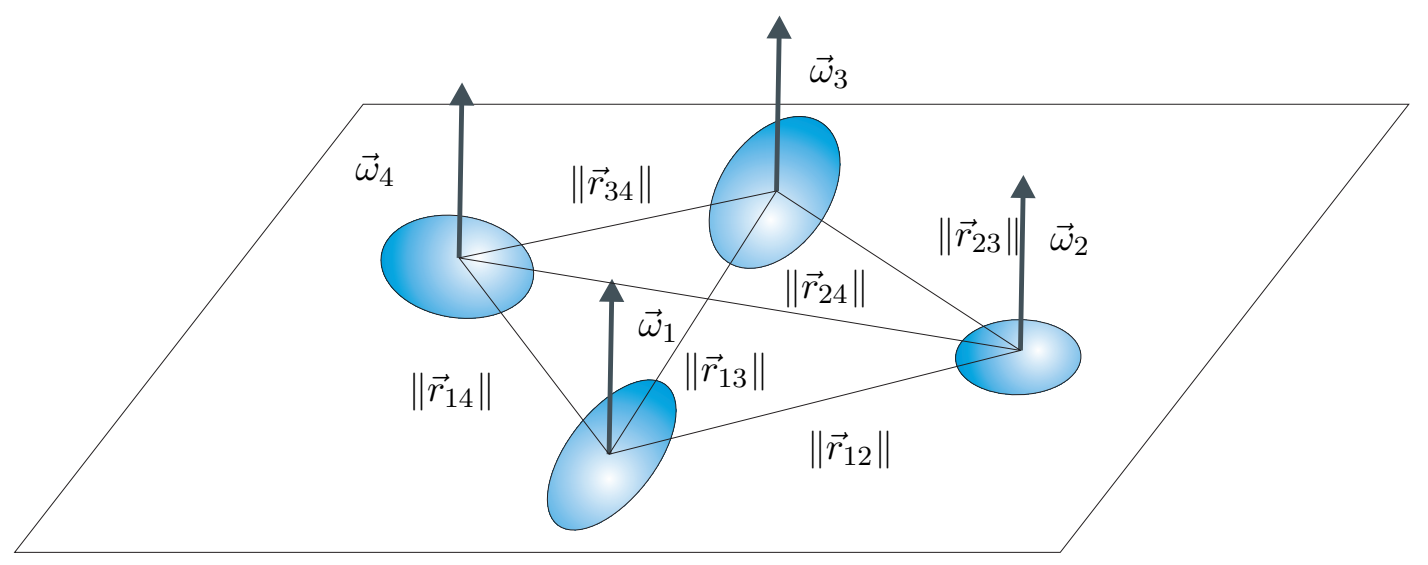

Figura 3.5: Solução sem perda de energia. 


\section{Propostas de Estudo}

Propusemos um modelo simplificado para a abordagem de dissipação de energia mecânica em corpos celestes. Tomamos o cuidado em identificá-lo como um caso restrito do problema geral, através de um vínculo. Para isto, foi essencial adotarmos a formulação Lagrangiana. Fomos capazes de comparar com certa proximidade as informações qualitativas e quantitativas do referido modelo com os resultados obtidos por trabalhos já extensivamente estudados.

Ressaltamos que o desenvolvimento do problema do corpo isolado, equações (3.24) e (3.26), foi o mais importante, no sentido de que o início do trabalho sobre o sistema de dois corpos, apresentado no texto, depende essencialmente deste primeiro modelo, assim como demais questões sobre este caso, as quais ainda serão exploradas futuramente. Esta fácil adaptação se deve à simplicidade que o modelo adquiriu. Ele permite rotações e deformações em direções arbitrárias do espaço, sendo que a equação (3.24) se manteve linear em $B$, e mesmo assim o momento angular do sistema persistiu como integral primeira e a energia persistiu como função de Lyapounov. Para isso, foi essencial termos tomado a aproximação na Lagrangiana, não nas equações de movimento.

Além disso, como mostrado no texto, trabalhos sobre sistemas com mais corpos já possuem sua formulação estabelecida neste contexto, dando início a diversas questões importantes, como equilíbrios, estabilidade e possivelmente capturas em ressonâncias. Aliás as propostas de estudo vão nesta linha.

- Para o problema de dois corpos, ficaram questões não respondidas sobre a estabilidade das órbitas circulares de equilíbrio. Para cada nível de momento angular total, vimos que o sistema admite pelo menos duas delas. Temos a impressão que somente a externa é estável. Mesmo respondida esta pergunta, seria interessante estimar sua bacia de atração. Temos algumas simulações que indicam que esta região de atração é considerável, mas não incluímos neste trabalho.

- Outro problema é encontrar uma estimativa para a segunda constante efetiva $\nu$ de (3.24). No sistema Terra-Lua, temos medidas precisas do afastamento médio da Lua, $3,82 \mathrm{~cm} /$ ano [BR99]. Podemos comparar esta medida com outros fenômenos também ligados à dissipação de energia na Terra. Este trabalho está em andamento.

- Ainda para dois corpos, há evidências numéricas de que há captura em ressonância spinórbita. Sabe-se que o próprio modelo de F. Mignard [Mig79] tem esta propriedade, quando acoplado à velocidade angular do corpo, ver [RCJMH13]. Uma proposta é verificar se o modelo apresentado aqui oferece mais informações sobre este fenômeno.

- Para o problema de $N$-corpos, vimos que a equação dos equilíbrios relativos (D.1) é uma perturbação da equação de configurações centrais. Usando as equações de Andoyer (ver [MF13]) é possível mostrar que $N$ corpos idênticos nos vértices de um $N$-ágono regular satisfazem estas equações, para qualquer $N$. Uma questão é entender quais outras classes de configurações centrais persistem por esta perturbação. 


\section{Apêndice A}

\section{Potencial Gravitacional}

Calculemos a integral usada na Proposição 2.6:

$$
I=\int_{0}^{\infty} \frac{\lambda}{\left(x^{-4}+\lambda\right)^{\frac{3}{2}}\left(x^{2}+\lambda\right)^{2}} d \lambda
$$

onde $x>1$. Faça a mudança de variáveis: $u=\lambda+x^{-4}$ para obter

$$
I=\int_{x^{-4}}^{\infty} \frac{1}{u^{\frac{1}{2}}\left(u+x^{2}-x^{-4}\right)^{2}} d u-x^{-4} \int_{x^{-4}}^{\infty} \frac{1}{u^{\frac{3}{2}}\left(u+x^{2}-x^{-4}\right)^{2}} d u=I_{1}-x^{-4} I_{2} .
$$

Em $I_{1}$, faça a mudança de coordenadas, $u=\left(x^{2}-x^{-4}\right) \tan ^{2} \theta$, para obter

$$
I_{1}=\frac{2 x^{6}}{\left(x^{6}-1\right)^{\frac{3}{2}}} \int_{a}^{b} \cos ^{2} \theta d \theta=\left.\frac{x^{6}}{\left(x^{6}-1\right)^{\frac{3}{2}}}\left(\theta+\frac{1}{2} \operatorname{sen}(2 \theta)\right)\right|_{a} ^{b},
$$

onde $a=\tan ^{-1}\left(\left(x^{6}-1\right)^{-\frac{1}{2}}\right)$ e $b=\pi / 2$. Logo, usando identidades trigonométricas,

$$
I_{1}=\frac{x^{6}}{\left(x^{6}-1\right)^{\frac{3}{2}}}\left(\frac{\pi}{2}-\tan ^{-1}\left(\left(x^{6}-1\right)^{-\frac{1}{2}}\right)-\frac{\sqrt{x^{6}-1}}{x^{6}}\right) .
$$

Agora, usando a mesma mudança de variáveis de $I_{1}$, obtemos

$$
\begin{gathered}
I_{2}=\frac{2 x^{10}}{\left(x^{6}-1\right)^{\frac{5}{2}}} \int_{a}^{b}\left(\operatorname{sen}^{2} \theta+\left(\operatorname{sen}^{2} \theta\right)^{-1}-2\right) d \theta=\left.\frac{2 x^{10}}{\left(x^{6}-1\right)^{\frac{5}{2}}}\left(-\frac{3}{2} \theta-\frac{1}{4} \operatorname{sen}(2 \theta)-\cot \theta\right)\right|_{a} ^{b} \\
\Rightarrow I_{2}=\frac{x^{10}}{\left(x^{6}-1\right)^{\frac{5}{2}}}\left(-\frac{3 \pi}{2}+3 \tan ^{-1}\left(\left(x^{6}-1\right)^{-\frac{1}{2}}\right)+\left(2 x^{6}+1\right) \frac{\sqrt{x^{6}-1}}{x^{6}}\right) .
\end{gathered}
$$

Logo,

$$
\begin{gathered}
I=\frac{x^{6}}{\left(x^{6}-1\right)^{\frac{5}{2}}}\left(\frac{x^{6}+2}{2} \pi-\left(x^{6}+2\right) \tan ^{-1}\left(\left(x^{6}-1\right)^{-\frac{1}{2}}\right)-3 \sqrt{x^{6}-1}\right) . \\
I=\frac{x^{6}}{\left(x^{6}-1\right)^{\frac{5}{2}}}\left(\left(x^{6}+2\right) \cot ^{-1}\left(\left(x^{6}-1\right)^{-\frac{1}{2}}\right)-3 \sqrt{x^{6}-1}\right) .
\end{gathered}
$$


APÊNDICE A 


\section{Apêndice B}

\section{Aproximação do Potencial Gravitacional}

\section{B.1 Densidade homogênea}

O potencial gravitacional de um corpo esférico, de raio $R$, deformado linearmente por uma matriz $A \in \mathrm{SSym}_{+}(3)$ é dado por:

$$
V(A)=-\frac{G \rho^{2}}{2} \int_{\mathcal{B}} \int_{\mathcal{B}} \frac{1}{\|A(x-y)\|} d x d y .
$$

Consideremos a expansão em polinômio de Taylor desta função, até segunda ordem, na variável $B(\operatorname{com} A=\exp \varepsilon B)$

$$
V(A)=\phi(\varepsilon B):=-\frac{G \rho^{2}}{2} \int_{\mathcal{B}} \int_{\mathcal{B}} \frac{1}{\left\|e^{\varepsilon B}(x-y)\right\|} d x d y .
$$

Assim (escolhendo $\phi(0)=0$ ),

$$
\phi(\varepsilon B)=\varepsilon D \phi(0) B+\frac{\varepsilon^{2}}{2} D^{2} \phi(0) B^{2}+\mathcal{O}\left(\varepsilon^{3}\right) .
$$

Note que a derivada do integrando, com respeito a $B$, ainda é integrável. Logo, podemos derivar sob o sinal de integral. Além disso, como as integrais são em bolas, podemos supor que $B$ é diagonal (dentro da integral)

$2 D \phi(0) B=\left.2 \frac{d}{d \lambda}\right|_{\lambda=0} \phi(\lambda B)=G \rho^{2} \int_{\mathcal{B}} \int_{\mathcal{B}} \frac{\langle x-y, B(x-y)\rangle}{\|x-y\|^{3}} d x d y=G \rho^{2} \sum_{i=1}^{3} b_{i} \int_{\mathcal{B}} \int_{\mathcal{B}} \frac{\left(x_{i}-y_{i}\right)^{2}}{\|x-y\|^{3}} d x d y$,

onde $b_{i}$ são os autovalores de $B$. Note que as integrais dos termos $\left(x_{i}-y_{i}\right)\left(x_{j}-y_{j}\right)$, para $i \neq j$, se anulam. Ainda, pela invariância da integral por rotações, concluímos que todas as integrais da última expressão são iguais. Logo,

$$
\begin{gathered}
D \phi(0) B=\alpha \operatorname{Tr} B=0, \\
2 D^{2} \phi(0) B^{2}=\left.\left.2 \frac{d}{d \beta}\right|_{\beta=0} \frac{d}{d \lambda}\right|_{\lambda=0} \phi((\lambda+\beta) B)=\left.\frac{d}{d \beta}\right|_{\beta=0} G \rho^{2} \int_{\mathcal{B}} \int_{\mathcal{B}} \frac{\left\langle e^{\beta B}(x-y), e^{\beta B} B(x-y)\right\rangle}{\left\|e^{\beta B}(x-y)\right\|^{3}} d x d y \\
=G \rho^{2}\left(2 \int_{\mathcal{B}} \int_{\mathcal{B}} \frac{\|B(x-y)\|^{2}}{\|x-y\|^{3}} d x d y-3 \int_{\mathcal{B}} \int_{\mathcal{B}} \frac{\langle x-y, B(x-y)\rangle^{2}}{\|x-y\|^{5}} d x d y\right) .
\end{gathered}
$$

É simples verificar que todas as funções que ocorrem na expressão acima são de fato integráveis 
nestes domínios.

Novamente, considerando $B$ diagonal, as simetrias das integrais com respeito a rotações e com algumas manipulações, concluímos que

$$
2 D^{2} \phi(0) B^{2}=G \rho^{2}\left(2 h_{0}-3 h_{1}+3 h_{2}\right) \operatorname{Tr}\left(B^{2}\right),
$$

onde,

$$
\begin{gathered}
h_{0}:=\int_{\mathcal{B}} \int_{\mathcal{B}} \frac{\left(x_{i}-y_{i}\right)^{2}}{\|x-y\|^{3}} d x d y \quad h_{1}:=\int_{\mathcal{B}} \int_{\mathcal{B}} \frac{\left(x_{i}-y_{i}\right)^{4}}{\|x-y\|^{5}} d x d y \quad i=1,2,3, \\
h_{2}:=\int_{\mathcal{B}} \int_{\mathcal{B}} \frac{\left(x_{i}-y_{i}\right)^{2}\left(x_{j}-y_{j}\right)^{2}}{\|x-y\|^{5}} d x d y \quad i \neq j .
\end{gathered}
$$

Observe agora que,

$$
\begin{gathered}
3 h_{0}=\int_{\mathcal{B}} \int_{\mathcal{B}} \frac{\sum_{i=1}^{3}\left(x_{i}-y_{i}\right)^{2}}{\|x-y\|^{3}} d x d y=\int_{\mathcal{B}} \int_{\mathcal{B}} \frac{1}{\|x-y\|} d x d y, \\
3 h_{1}+6 h_{2}=\int_{\mathcal{B}} \int_{\mathcal{B}} \frac{\left(\sum_{i=1}^{3}\left(x_{i}-y_{i}\right)^{2}\right)^{2}}{\|x-y\|^{5}} d x d y=\int_{\mathcal{B}} \int_{\mathcal{B}} \frac{1}{\|x-y\|} d x d y .
\end{gathered}
$$

Logo,

$$
2 D^{2} \phi(0) B^{2}=G \rho^{2}\left(2 h_{0}-3 h_{1}+\frac{3}{2}\left(h_{0}-h_{1}\right)\right) \operatorname{Tr}\left(B^{2}\right)=\frac{1}{2} G \rho^{2}\left(7 h_{0}-9 h_{1}\right) \operatorname{Tr}\left(B^{2}\right) .
$$

Mas, a expressão para a última integral é bem conhecida:

$$
\int_{\mathcal{B}} \int_{\mathcal{B}} \frac{1}{\|x-y\|} d x d y=\frac{32}{15} \pi^{2} R^{5}
$$

Proposição B.1. Afirmação:

$$
h_{1}=\frac{32}{75} \pi^{2} R^{5}
$$

Demonstração. Considere a seguinte integral em coordenadas esféricas (tomando $y \in \mathcal{B}-\{0\}$ ):

$$
\begin{gathered}
\int_{\mathcal{B}}\|x-y\|^{3} d x=\int_{0}^{R} \int_{0}^{\pi} \int_{0}^{2 \pi}\left(r^{2}+\|y\|^{2}-2\|y\| r \cos \theta\right)^{\frac{3}{2}} r^{2} \operatorname{sen} \theta \quad d \varphi d \theta d r= \\
=\frac{2 \pi}{5\|y\|} \int_{0}^{R} r\left(\int_{0}^{\pi} \frac{\partial}{\partial \theta}\left(r^{2}+\|y\|^{2}-2\|y\| r \cos \theta\right)^{\frac{5}{2}} d \theta\right) d r= \\
=\frac{2 \pi}{5\|y\|}\left(\int_{0}^{\|y\|} r\left((r+\|y\|)^{5}-(\|y\|-r)^{5}\right) d r+\int_{\|y\|}^{R} r\left((r+\|y\|)^{5}-(r-\|y\|)^{5}\right) d r\right)= \\
=\frac{2 \pi}{5\|y\|}\left(\int_{0}^{\|y\|} 2 r^{6}+20\|y\|^{2} r^{4}+10\|y\|^{4} r^{2} d r+\int_{\|y\|}^{R} 10\|y\| r^{5}+20\|y\|^{3} r^{3}+2\|y\|^{5} r d r\right) \\
\Rightarrow \int_{\mathcal{B}}\|x-y\|^{3} d x=\frac{2 \pi}{5}\left(-\frac{1}{21}\|y\|^{6}+\frac{5}{3} R^{6}+5 R^{4}\|y\|^{2}+R^{2}\|y\|^{4}\right) .
\end{gathered}
$$

Além disso, verifica-se facilmente que:

$$
\frac{\partial^{4}}{\partial y_{i}^{4}}\|x-y\|^{3}=9\left(\frac{1}{\|x-y\|}-2 \frac{\left(x_{i}-y_{i}\right)^{2}}{\|x-y\|^{3}}+\frac{\left(x_{i}-y_{i}\right)^{4}}{\|x-y\|^{5}}\right) .
$$


Sendo todos os termos envolvidos neste cálculo integráveis em $\mathcal{B}$. Logo,

$$
\int_{\mathcal{B}} \frac{\left(x_{i}-y_{i}\right)^{4}}{\|x-y\|^{5}} d x=\frac{1}{9} \frac{\partial^{4}}{\partial y_{i}^{4}} \int_{\mathcal{B}}\|x-y\|^{3} d x-\int_{\mathcal{B}} \frac{1}{\|x-y\|} d x+2 \int_{\mathcal{B}} \frac{\left(x_{i}-y_{i}\right)^{2}}{\|x-y\|^{3}} d x .
$$

Para obter $h_{1}$, basta integrar esta expressão. Mas note que, novamente pelas simetrias da integral,

$$
\begin{gathered}
\int_{\mathcal{B}} \int_{\mathcal{B}} \frac{\left(x_{i}-y_{i}\right)^{2}}{\|x-y\|^{3}} d x d y=\frac{1}{3} \int_{\mathcal{B}} \int_{\mathcal{B}} \frac{\|x-y\|^{2}}{\|x-y\|^{3}} d x d y=\frac{1}{3} \int_{\mathcal{B}} \int_{\mathcal{B}} \frac{1}{\|x-y\|} d x d y \\
\Rightarrow h_{1}=\frac{1}{9} \int_{\mathcal{B}}\left(\frac{\partial^{4}}{\partial y_{i}^{4}} \int_{\mathcal{B}}\|x-y\|^{3} d x\right) d y-\frac{1}{3} \int_{\mathcal{B}} \int_{\mathcal{B}} \frac{1}{\|x-y\|} d x d y .
\end{gathered}
$$

Mas, de (B.4), temos

$$
\int_{\mathcal{B}}\left(\frac{\partial^{4}}{\partial y_{i}^{4}} \int_{\mathcal{B}}\|x-y\|^{3} d x\right) d y=\frac{2 \pi}{5} \int_{\mathcal{B}}\left(24\left(-\frac{4}{7} y_{i}^{2}-\frac{1}{7}\|y\|^{2}+R^{2}\right)\right) d y
$$

Sabendo que,

$$
\begin{gathered}
3 \int_{\mathcal{B}} y_{i}^{2} d y=\int_{\mathcal{B}}\|y\|^{2} d y=2 \pi \int_{0}^{R} \int_{0}^{\pi} r^{4} \operatorname{sen} \theta d \theta d r=\frac{4 \pi R^{5}}{5} \\
\Rightarrow \int_{\mathcal{B}}\left(\frac{\partial^{4}}{\partial y_{i}^{4}} \int_{\mathcal{B}}\|x-y\|^{3} d x\right) d y=\frac{48 \pi}{5}\left(-\frac{1}{3} \frac{4 \pi R^{5}}{5}+\frac{4 \pi R^{5}}{3}\right)=\frac{256}{25} \pi^{2} R^{5} .
\end{gathered}
$$

Portanto,

$$
h_{1}=\frac{32}{75} \pi^{2} R^{5}
$$

Desta forma, de (B.2), concluímos que:

$$
\phi(\varepsilon B)=\frac{2}{25} \times \frac{16}{9} G \pi^{2} \rho^{2} R^{5} \varepsilon^{2} \operatorname{Tr}\left(B^{2}\right)+\mathcal{O}\left(\varepsilon^{3}\right)=\frac{2}{25} \frac{G M^{2}}{R} \varepsilon^{2} \operatorname{Tr}\left(B^{2}\right)+\mathcal{O}\left(\varepsilon^{3}\right) .
$$

\section{B.2 Densidade não-homogênea}

Suponhamos agora que o corpo possua densidade de massa não-homogênea, mas esfericamente simétrica, ou seja, que dependa somente da distância ao centro de massa (assim como em [KVG $\left.{ }^{+} 12\right]$ ). Neste caso, a energia potencial se torna

$$
V(A)=-\frac{G}{2} \int_{\mathcal{B}} \rho(\|y\|) \int_{\mathcal{B}} \frac{\rho(\|x\|)}{\|A(x-y)\|} d x d y .
$$

Note que com esta hipótese, a maioria das considerações da seção anterior continuam verdadeiras, inclusive que $D \phi(0) B=0$ e

$$
D^{2} \phi(0) B^{2}=\frac{1}{4} G\left(7 h_{0}-9 h_{1}\right) \operatorname{Tr}\left(B^{2}\right)
$$

onde

$$
h_{0}:=\frac{1}{3} \int_{\mathcal{B}} \rho(\|y\|) \int_{\mathcal{B}} \frac{\rho(\|x\|)}{\|x-y\|} d x d y \quad h_{1}:=\int_{\mathcal{B}} \rho(\|y\|) \int_{\mathcal{B}} \rho(\|x\|) \frac{\left(x_{i}-y_{i}\right)^{4}}{\|x-y\|^{5}} d x d y \quad i=1,2,3 .
$$


Calculemos o fator $h_{0}$. Seja,

$$
E(y):=\nabla_{y} \int_{\mathcal{B}} \frac{\rho(\|x\|)}{\|x-y\|} d x=-\int_{\mathcal{B}} \rho(\|x\|) \frac{x-y}{\|x-y\|^{3}} d x .
$$

Como usual, pela simetria desta integral pelas rotações que fixam $y$, concluímos que $E(y)=$ $\lambda(\|y\|) y$. Assim, pelo teorema de Gauss, seu fluxo em uma esfera de raio $r_{1}$ é:

$$
\int_{S_{r_{1}}}\left\langle E(y), \hat{n}_{y}\right\rangle d S=4 \pi r_{1}^{3} \lambda\left(r_{1}\right)=\int_{B_{r_{1}}} \operatorname{div} E(y) d y=4 \pi \int_{B_{r_{1}}} \rho(\|y\|) d y=16 \pi^{2} \int_{0}^{r_{1}} \rho(s) s^{2} d s .
$$

Logo,

$$
E(y)=\frac{4 \pi}{\|y\|^{3}}\left(\int_{0}^{\|y\|} \rho(s) s^{2} d s\right) y .
$$

Decorre que $E(y)=\nabla \chi(y)$, sendo

$$
\chi(y)=4 \pi \int_{0}^{\|y\|} \frac{\int_{0}^{u} \rho(s) s^{2} d s}{u^{2}} d u .
$$

Disto, concluímos que

$$
\int_{\mathcal{B}} \frac{\rho(\|x\|)}{\|x-y\|} d x=4 \pi\left(\int_{0}^{R} \rho(s) s d s-\int_{0}^{\|y\|} \frac{\int_{0}^{u} \rho(s) s^{2} d s}{u^{2}} d u\right) .
$$

Assim,

$$
h_{0}=\frac{16 \pi^{2}}{3}\left(\left(\int_{0}^{R} \rho(s) s d s\right)\left(\int_{0}^{R} \rho(s) s^{2} d s\right)-\int_{0}^{R} r^{2} \rho(r)\left(\int_{0}^{r} \frac{\int_{0}^{u} \rho(s) s^{2} d s}{u^{2}} d u\right) d r\right) .
$$

Já $h_{1}$ não admite uma expressão tão simples. Analogamente a (B.5), temos

$$
h_{1}=\frac{1}{9} \int_{\mathcal{B}} \rho(\|y\|) \frac{\partial^{4}}{\partial y_{i}^{4}}\left(\int_{\mathcal{B}} \rho(\|x\|)\|x-y\|^{3} d x\right) d y-h_{0} .
$$

Para o caso particular de duas camadas de densidade homogênea, núcleo e manto, devemos tomar a densidade:

$$
\rho(y)=\left\{\begin{array}{l}
\rho_{1}, \quad\|y\| \leq R_{1}, \\
\rho_{2}, \quad R_{1}<\|y\|<R_{2} .
\end{array}\right.
$$

Calculando as integrais em (B.8), obtemos:

$$
h_{0}=\frac{16 \pi^{2}}{45}\left(2 \rho_{2}^{2} R_{2}^{5}+\left(\rho_{1}-\rho_{2}\right)\left(2 \rho_{1}-3 \rho_{2}\right) R_{1}^{5}+5 \rho_{2}\left(\rho_{1}-\rho_{2}\right) R_{1}^{3} R_{2}^{2}\right) .
$$

Também usando a expressão (B.4), obtemos que

$$
\begin{gathered}
I:=\int_{\mathcal{B}} \rho(\|x\|)\|x-y\|^{3} d x=\int_{\mathcal{B}_{R_{1}}}\left(\rho_{1}-\rho_{2}\right)\|x-y\|^{3} d x+\int_{\mathcal{B}_{R_{2}}} \rho_{2}\|x-y\|^{3} d x \\
=\frac{2 \pi}{5}\left(\left(\rho_{1}-\rho_{2}\right)\left(-\frac{1}{21}\|y\|^{6}+\frac{5}{3} R_{1}^{6}+5 R_{1}^{4}\|y\|^{2}+R_{1}^{2}\|y\|^{4}\right)+\rho_{2}\left(-\frac{1}{21}\|y\|^{6}+\frac{5}{3} R_{2}^{6}+5 R_{2}^{4}\|y\|^{2}+R_{2}^{2}\|y\|^{4}\right)\right) .
\end{gathered}
$$

Desta forma, de (B.9)

$$
h_{1}=\frac{1}{9}\left(\frac{64 \pi^{2}}{25}\right)\left(4\left(\rho_{1}-\rho_{2}\right)^{2} R_{1}^{5}+4 \rho_{2}^{2} R_{2}^{5}+\rho_{2}\left(\rho_{1}-\rho_{2}\right)\left(5 R_{1}^{2} R_{2}^{2}\left(R_{1}+R_{2}\right)-\left(R_{1}^{5}+R_{2}^{5}\right)\right)-h_{0} .\right.
$$


Observe que estas expressões se reduzem ao caso anterior ao tomarmos $\rho_{1}=\rho_{2}$. 
APÊNDICE B 


\section{Apêndice C}

\section{Integral no problema de dois corpos}

Queremos calcular

$$
\int_{\mathcal{B}} \frac{(\vec{r}-x) x^{T}}{\|\vec{r}-x\|^{3}} d x
$$

Considere a seguinte integral em coordenadas esféricas (tomando $y \notin \mathcal{B}$ ):

$$
\begin{gathered}
\int_{\mathcal{B}}\|y-x\| d x=\int_{0}^{R} \int_{0}^{\pi} \int_{0}^{2 \pi}\left(r^{2}+\|y\|^{2}-2\|y\| r \cos \theta\right)^{\frac{1}{2}} r^{2} \operatorname{sen} \theta d \varphi d \theta d r= \\
=\frac{2 \pi}{3\|y\|} \int_{0}^{R} r\left(\int_{0}^{\pi} \frac{\partial}{\partial \theta}\left(r^{2}+\|y\|^{2}-2\|y\| r \cos \theta\right)^{\frac{3}{2}} d \theta\right) d r= \\
=\frac{2 \pi}{3\|y\|}\left(\int_{0}^{R} r\left((r+\|y\|)^{3}-(\|y\|-r)^{3}\right) d r\right)=\frac{4 \pi}{3}\left(\frac{R^{5}}{5\|y\|}+R^{3}\|y\|\right) .
\end{gathered}
$$

Definindo-se assim,

$$
G(\vec{r}):=\frac{4 \pi}{3}\left(\frac{R^{5}}{5\|\vec{r}\|}+R^{3}\|\vec{r}\|\right)
$$

decorre que:

$$
\begin{gathered}
\frac{4 \pi}{3} R^{3} \frac{\vec{r}}{\|\vec{r}\|}-\int_{\mathcal{B}} \frac{x}{\|\vec{r}-x\|} d x=\int_{\mathcal{B}} \frac{\vec{r}-x}{\|\vec{r}-x\|} d x=\nabla G(\vec{r})=\frac{4 \pi}{3}\left(-\frac{R^{5}}{5\|\vec{r}\|^{3}}+\frac{R^{3}}{\|\vec{r}\|}\right) \vec{r} \\
\Rightarrow \int_{\mathcal{B}} \frac{x}{\|\vec{r}-x\|} d x=\frac{4 \pi}{15} \frac{R^{5}}{\|\vec{r}\|^{3}} \vec{r}=:\left(U_{1}(\vec{r}), U_{2}(\vec{r}), U_{3}(\vec{r})\right) .
\end{gathered}
$$

Definidas desta forma, as funções $U_{i}$ satisfazem:

$$
\int_{\mathcal{B}} \frac{x_{i}(\vec{r}-x)}{\|\vec{r}-x\|^{3}} d x=-\nabla U_{i}(\vec{r})=-\frac{4 \pi}{15} \frac{R^{5}}{\|\vec{r}\|^{3}} \vec{e}_{i}+\frac{4 \pi}{5} \frac{R^{5}}{\|\vec{r}\|^{5}} r_{i} \vec{r} .
$$

Portanto,

$$
\int_{\mathcal{B}} \frac{(\vec{r}-x) x^{T}}{\|\vec{r}-x\|^{3}} d x=-\frac{4 \pi}{15} \frac{R^{5}}{\|\vec{r}\|^{3}} \operatorname{Id}+\frac{4 \pi}{5} \frac{R^{5}}{\|\vec{r}\|^{5}} \vec{r} \vec{r}^{T}
$$


APÊNDICE C 


\section{Apêndice D}

\section{Campo de forças no problema de $\mathrm{N}$ corpos}

Na Seção 3.7, ao tratar as soluções conservativas do problema de N-corpos, supusemos que cada maré induzida em cada corpo é constante. Assim, garantimos que todos os corpos devem estar em um mesmo plano, com velocidade angular perpendicular ao mesmo. Queremos garantir que neste caso específico, o campo de forças de (3.107) é composto de pares de forças opostas, de mesma intensidade, na direção que une os correspondentes pares de corpos, desta forma o chamaremos de central.

Na referida equação, os três primeiros termos são centrais. No último termo, usando a equação (3.105), teremos a contribuição de $\Omega^{2}, \operatorname{Tr}\left(\Omega^{2}\right) \operatorname{Id~e~}\left(M_{j} / r_{i j}^{3}\right)$ Id, aplicadas em $\vec{r}_{i j}$, termos que também são centrais. Analisemos, portanto, o termo advindo de $R_{i}^{2} Y_{i} B_{i} Y_{i}^{T} \vec{r}_{i j}$

$$
\begin{aligned}
\sum_{j \neq i} \frac{6 G M_{j} R_{i}^{2} \varepsilon^{\frac{8}{3}}}{5 r_{i j}^{5}} \sum_{k \neq i} \frac{3 G M_{k}}{r_{i k}^{5}} & \left\langle\vec{r}_{i k}, \vec{r}_{i j}\right\rangle \vec{r}_{i k} \\
& =\sum_{j \neq i} \frac{18 G^{2} M_{j} R_{i}^{2} \varepsilon^{\frac{8}{3}}}{5 r_{i j}^{5}}\left(\sum_{\substack{k \neq i \\
k \neq j}} \frac{M_{k}}{r_{i k}^{5}}\left\langle\vec{r}_{i k}, \vec{r}_{i j}\right\rangle\right) \vec{r}_{i j}+\sum_{j \neq i} \frac{18 G^{2} M_{j}^{2} R_{i}^{2} \varepsilon^{\frac{8}{3}}}{5 r_{i j}^{8}} \vec{r}_{i j},
\end{aligned}
$$

onde fizemos uma troca de índices. O termo advindo de $R_{j}^{2} Y_{j} B_{j} Y_{j}^{T} \vec{r}_{i j}$ fica

$$
\begin{aligned}
& \sum_{j \neq i} \frac{6 G M_{j} R_{j}^{2} \varepsilon^{\frac{8}{3}}}{5 r_{i j}^{5}} \sum_{k \neq j} \frac{3 G M_{k}}{r_{j k}^{5}}\left\langle\vec{r}_{j k}, \vec{r}_{i j}\right\rangle\left(-\vec{r}_{i j}+\vec{r}_{i k}\right)=\sum_{j \neq i} \frac{18 G^{2} M_{j} M_{i} R_{j}^{2} \varepsilon^{\frac{8}{3}}}{5 r_{i j}^{8}} \vec{r}_{i j} \\
& \quad-\sum_{j \neq i} \frac{18 G^{2} M_{j} R_{j}^{2} \varepsilon^{\frac{8}{3}}}{5 r_{i j}^{5}}\left(\sum_{\substack{k \neq i \\
k \neq j}} \frac{M_{k}}{r_{j k}^{5}}\left\langle\vec{r}_{j k}, \vec{r}_{i j}\right\rangle\right) \vec{r}_{i j}-\sum_{j \neq i} \frac{18 G^{2} M_{j} \varepsilon^{\frac{8}{3}}}{5}\left(\sum_{\substack{k \neq i \\
k \neq j}} \frac{M_{k} R_{k}^{2}}{r_{j k}^{5} r_{i k}^{5}}\left\langle\vec{r}_{j k}, \vec{r}_{i k}\right\rangle\right) \vec{r}_{i j} .
\end{aligned}
$$


Assim, multiplicando as duas equações acima por $M_{i}$ e somando-as, obtemos

$$
\begin{array}{r}
\sum_{j \neq i} \frac{18 G^{2} M_{j} M_{i}\left(M_{i} R_{j}^{2}+M_{j} R_{i}^{2}\right) \varepsilon^{\frac{8}{3}}}{5 r_{i j}^{8}} \vec{r}_{i j}+\sum_{j \neq i} \frac{18 G^{2} M_{i} M_{j} \varepsilon^{\frac{8}{3}}}{5 r_{i j}^{5}}\left(\sum_{\substack{k \neq i \\
k \neq j}} M_{k}\left\langle\frac{R_{i}^{2}}{r_{i k}^{5}} \vec{r}_{i k}-\frac{R_{j}^{2}}{r_{j k}^{5}} \vec{r}_{j k}, \vec{r}_{i j}\right\rangle\right) \vec{r}_{i j} \\
+\sum_{j \neq i} \frac{18 G^{2} M_{i} M_{j} \varepsilon^{\frac{8}{3}}}{5}\left(\sum_{\substack{k \neq i \\
k \neq j}} \frac{M_{k} R_{k}^{2}}{r_{j k}^{5} r_{i k}^{5}}\left\langle\vec{r}_{i k}, \vec{r}_{k j}\right\rangle\right) \vec{r}_{i j} .
\end{array}
$$

Observe que todos os coeficientes de $\vec{r}_{i j}$ são simétricos, portanto esta parte da força também é central, provando o que pretendíamos.

Explicitamente, verificamos que a equação (3.107) fica

$$
\begin{aligned}
& -M_{i} \omega^{2} \vec{P}_{i}=-\sum_{j \neq i} \frac{G M_{i} M_{j} \varepsilon}{r_{i j}^{3}} \vec{r}_{i j}-\sum_{j \neq i} \frac{3 G M_{i} M_{j} \varepsilon^{\frac{8}{3}}}{5 r_{i j}^{5}}\left(\left(R_{i}^{2}+R_{j}^{2}\right) \omega^{2}-\sum_{\substack{k \neq i \\
k \neq j}} 3 G M_{k}\left(\frac{R_{j}^{2}}{r_{j k}^{3}}+\frac{R_{i}^{2}}{r_{i k}^{3}}\right)\right) \vec{r}_{i j} \\
& -\sum_{j \neq i} \frac{9 G^{2} M_{i} M_{j} \varepsilon^{\frac{8}{3}}}{r_{i j}^{7}}\left(\frac{2\left(M_{j} R_{i}^{2}+M_{i} R_{j}^{2}\right)}{5 r_{i j}}+\sum_{\substack{k \neq i \\
k \neq j}} M_{k}\left(\frac{R_{i}^{2}\left\langle\vec{r}_{i k}, \vec{r}_{i j}\right\rangle^{2}}{r_{i k}^{5}}+\frac{R_{j}^{2}\left\langle\vec{r}_{j k}, \vec{r}_{i j}\right\rangle^{2}}{r_{j k}^{5}}\right)\right) \vec{r}_{i j} \\
& \quad+\sum_{j \neq i} \frac{18}{5} G^{2} M_{j} M_{i} \varepsilon^{\frac{8}{3}}\left(\sum_{\substack{k \neq i \\
k \neq j}} \frac{M_{k}}{r_{i j}^{5}}\left\langle\frac{R_{i}^{2}}{r_{i k}^{5}} \vec{r}_{i k}-\frac{R_{j}^{2}}{r_{j k}^{5}} \vec{r}_{j k}, \vec{r}_{i j}\right\rangle+\sum_{\substack{k \neq i \\
k \neq j}} \frac{M_{k} R_{k}^{2}}{r_{j k}^{5} r_{i k}^{5}}\left\langle\vec{r}_{i k}, \vec{r}_{k j}\right\rangle\right) \vec{r}_{i j} . \quad \text { (D.1) }
\end{aligned}
$$




\section{Apêndice E}

\section{Número de Love}

Neste apêndice, discutimos a diferença entre o número de Love $k_{2}$ usado por diversos autores (ver (3.33)) e a correspondente expressão que encontramos em (3.32).

A abordagem de [Lov44] consiste em considerar a defomação de uma bola sujeita a uma ação de forças elásticas e auto-gravitacionais. A parametrização da superfície deformada é

$$
r=R\left(1-\frac{2}{3} \varepsilon\left(\frac{3}{2} \cos ^{2} \theta-\frac{1}{2}\right)\right),
$$

onde o parâmetro $\varepsilon$ representa, em primeira ordem, a eliticidade do corpo, e $R$ é o raio da esfera de repouso. No Capítulo 3 usamos como parametrização para o corpo a matriz $A=e^{\varepsilon B_{0}}$, onde $\varepsilon$ também é (novamente em primeira ordem) a eliticidade do elipsoide, e

$$
B_{0}=\frac{1}{3}\left(\begin{array}{ccc}
1 & 0 & 0 \\
0 & 1 & 0 \\
0 & 0 & -2
\end{array}\right) .
$$

Ainda em [Lov44], a expressão para tal deformação, expandida até segunda ordem, é

$$
\vec{u}(x, y, z)=-A_{2} r^{2} \nabla p_{2}-B_{2} p_{2} \vec{r}-\nabla \phi_{2},
$$

onde $p_{2}, \phi_{2}$ são harmônicos esféricos sólidos de ordem dois, com

$$
p_{2}=\frac{2}{3}\left(\frac{r}{R}\right)^{2}\left(\frac{3}{2} \cos ^{2} \theta-\frac{1}{2}\right)=\frac{1}{3 R^{2}}\left(2 z^{2}-x^{2}-y^{2}\right)
$$

As equações de movimento, ver (2.32), e a condição de contorno adotadas, além de div $\vec{u}=0$ implicam que $A_{2}=-5 B_{2} / 4$ e $2 \phi_{2}=-R^{2}\left(B_{2}+4 A_{2}\right) p_{2}$. Decorre que

$$
\begin{aligned}
\vec{u}(x, y, z)=-\frac{B_{2}}{6 R^{2}}\left(3 x^{3}+3 x y^{2}+9 x z^{2}-8 R^{2} x, 3 y^{3}+3 y x^{2}+9 y z^{2}-8 R^{2} y\right. & \\
& \left.-6 z^{3}-12 z x^{2}-12 z y^{2}+16 R^{2} z\right) .
\end{aligned}
$$

De (3.31) observamos que a eliticidade correspondente é $\varepsilon=5 B_{2} /\left(2\left(1+5 B_{2} / 6\right)\right) \approx 5 B_{2} / 2$. Lembrando da energia elástica definida em (2.26), ao impor a deformação (E.1) sua expressão fica

$$
E_{1}=\mu \int_{\mathcal{B}} \operatorname{Tr}\left(D \vec{u}^{2}\right)(\vec{x}) d \vec{x}=\left(\frac{4 \pi R^{3}}{3}\right)\left(\frac{2 \cdot 19}{3 \cdot 25} \mu\right) \varepsilon^{2} .
$$

No entanto, nós obtivemos a energia elástica (3.22)

$$
E_{2}=\mu \int_{\mathcal{B}} \operatorname{Tr}\left((\varepsilon B)^{2}\right) d \vec{x}=\left(\frac{4 \pi R^{3}}{3}\right)\left(\frac{2}{3} \mu\right) \varepsilon^{2} .
$$


Portanto podemos afirmar que nossa deformação superestima a energia elástica. Além disso, a razão entre as energias é $E_{1} / E_{2}=19 / 25$, a qual é exatamente a razão entre a contribuição elástica ao número de Love de cada modelo. 


\section{Referências Bibliográficas}

[ABC14] F. Antognini, L. Biasco e L. Chierchia. The spin-orbit resonances of the solar system: A mathematical treatment matching physical data. Journal of Nonlinear Science, 24:473-492, 2014.

[ACG08] H.M. Antia, S.M. Chitre e D.O. Gough. Temporal variations in the Sun's rotational kinetic energy. Astronomy and Astrophysics, 477:657-663, 2008.

[AMR07] R. Abraham, J. E. Marsden e T. Ratiu. Manifolds, Tensor Analysis and Applications. Springer-Verlag, New York, 3a edição, 2007.

[Arn66] V. I. Arnold. Sur la géométrie différentielle des groupes de Lie de dimension infinie et ses applications à l'hydrodynamique des fluides parfaits. Annales de l'Institut Fourier, 16:319-361, 1966.

[Arn89] V. I. Arnold. Mathematical Methods of Classical Mechanics. Springer, Berlin, segunda edição, 1989.

[Bat00] G. K. Batchelor. An Introduction to Fluid Mechanics. Cambridge University Press, Cambridge, 2000.

[BG06] N. Berglund e B. Gentz. Noise-Induced Phenomena in Slow-Fast Dynamical Systems. A Sample-Paths Approach. Springer, London, 2006.

[BH12] D. Bambusi e E. Haus. Asymptotic stability of synchronous orbits for a gravitating viscoelastic sphere. Celestial Mechanics and Dynamical Astronomy, 114:255-277, 2012 .

[BKM09] A. V. Borisov, A. A. Kilin e I. S. Mamaev. The Hamiltonian dynamics of selfgravitating liquid and gas ellipsoids. Regular and Chaotic Dynamics, 14:179-217, 2009 .

[BL87] J. Baillieul e M. Levi. Rotational elastic dynamics. Physica D: Nonlinear Phenomena, 27:43-62, 1987.

[BL91] J. Baillieul e M. Levi. Constrained relative motions in rotational mechanics. Archive For Rational Mechanics and Analysis, 115:101-135, 1991.

[BR99] B.G. Bills e R. D. Ray. Lunar orbital evolution: A synthesis of recent result. Geophysical Research Letters, 26:3045-3048, 1999.

[Car81] J. Carr. Applications of Centre Manifold Theory. Springer-Verlag, New York, 1981.

[CBLR14] A. C. M Correia, G. Boué, J. Laskar e A. Rodríguez. Deformation and tidal evolution of close-in planets and satellites using a Maxwell viscoelastic rheology. Astronomy $\mathscr{G}$ Astrophysics, 571, 2014.

[Cha87] S. Chandrasekhar. Ellipsoidal Figures of Equilibrium. Dover, New York, 1987. 
[CL03] A. Correia e J. Laskar. Different tidal torques on a planet with a dense atmosphere and consequences to the spin dynamics. Journal of Geophysical Research, 108, 2003.

[Dar86] G. H. Darwin. On Jacobi's figure of equilibrium for a rotating mass of fluid. Proceedings of the Royal Society of London, 41:319-336, 1886.

[Dar08] G. H. Darwin. Tidal friction and cosmogony, volume II. Cambridge University Press, New York, 1908.

[Efr00] M. Efroimsky. Precession of a freely rotating rigid body. Inelastic relaxation in the vicinity of poles. Journal of Mathematical Physics, 41:1854-1889, 2000.

[EL07] M. Efroimsky e V. Lainey. Physics of bodily tides in terrestrial planets and the appropriate scales of dynamical evolution. Journal of Geophysics Research, 12, 2007.

[EW09] M. Efroimsky e J. G. Williams. Tidal torques: A critical review of some techniques. Celestial Mechanics and Dynamical Astronomy, 104:257-289, 2009.

[Fit12] R. Fitzpatrick. An Introduction to Celestial Mechanics. Cambridge University Press, Cambridge, 2012.

[FL01] F. Fassó e D. Lewis. Stability properties of the Riemann ellipsoids. Archive for Rational Mechanics and Analysis, 158:259-292, 2001.

[FM03] W. Förstner e B. Moonen. A metric for covariance matrices. In Geodesy-The Challenge of the 3rd Millennium. Springer, 2003.

[FM13] S Ferraz-Mello. Tidal synchronization of close-in satellites and exoplanets. A rheophysical approach. Celestial Mechanics and Dynamical Astronomy, 116:109-140, 2013.

[FMGRR15] S. Ferraz-Mello, C. Grotta-Ragazzo e L. S. Ruiz. Dissipative Forces in Celestial Mechanics. Publicações Matemáticas do IMPA, Rio de Janeiro, 2015.

[Gol56] H. Goldstein. Classical Mechanics. Addison-Wesley, Cambridge, 1956.

[Hal80] J. K. Hale. Ordinary Differential Equations. Robert E. Krieger Publishing Company, New York, segunda edição, 1980.

[HSS09] D. D. Holm, T. Schmah e C. Stoica. Geometric Mechanics and Symmetry. Oxford University Press, New York, 2009.

$\left[\mathrm{KVG}^{+} 12\right]$ K. U. Kristiansen, M. Vereshchagin, K. Goździewski, P. L. Palmer e R. M. Roberts. The two-body problem of a pseudo-rigid body and a rigid sphere. Celestial Mechanics and Dynamical Astronomy, 112:169-190, 2012.

[KWM91] P. S. Krishnaprasad, L-S. Wang e J. H. Maddocks. Hamiltonian dynamics of a rigid body in a central gravitational field. Celestial Mechanics and Dynamical Astronomy, 50:349-386, 1991.

[Lam32] H. Lamb. Hydrodynamics. Cambridge Mathematical Library, Cambridge, sexta edição, 1932.

[Lam80] K . Lambeck. Earth's Variable Rotation: Geophysical Causes and Consequences. Cambridge University Press, Cambridge, 1980.

[LL86] L. D. Landau e E. M. Lifshitz. Theory of Elasticity. Pergamon Press, terceira edição, 1986.

[LL87] L. D. Landau e E. M. Lifshitz. Fluid Mechanics. Pergamon Press, segunda edição, 1987. 
[Lov44] A. E. H. Love. A Treatise on the Mathematical Theory of Elasticity. Dover, New York, 4a edição, 1944.

[MF13] L. F. Mello e A. C. Fernandes. Stacked central configurations for the spatial seven body problem. Qualitative Theory of Dynamical Systems, 12:101-114, 2013.

[Mig79] F. Mignard. The evolution of the lunar orbit revisited I. The Moon and The Planets, 20:301-315, 1979.

[Mun84a] R. G. Muncaster. Invariant manifolds in mechanics I: The general construction of coarse theories from fine theories. Archive for Rational Mechanics and Analysis, 84:353$373,1984$.

[Mun84b] R. G. Muncaster. Invariant manifolds in mechanics II: Zero-dimensional elastic bodies with directors. Archive for Rational Mechanics and Analysis, 84:375-392, 1984.

[NHFR13] N. Nettelmann, R. Helled, J.J. Fortney e R. Redmer. New indication for a dichotomy in the interior structure of Uranus and Neptune from the application of modified shape and rotation data. Planetary and Space Science, 77:143-151, 2013.

[NO84] T. R. Nordenholz e O. M. O'Reilly. On steady motions of isotropic, elastic Cosserat points. IMA Journal of Applied Mathematics, 60:55-72, 1984.

[Per01] L. Perko. Differential Equations and Dynamical Systems. Springer-Verlag, New York, 3a edição, 2001.

[RCJMH13] A. Rodríguez, N. Callegari Jr, T. A. Michtchenko e H. Hussmann. Spin-orbit coupling for tidally evolving super-Earths. MNRAS, 427:2239-2250, 2013.

[RD11] J.P. Rozelot e C. Damiani. History of solar oblateness measurements and interpretation. The European Physical Journal H, 36(3):407-436, 2011.

[RR15] C. Ragazzo e L. S. Ruiz. Dynamics of an isolated, viscoelastic, self-gravitating body. Celestial Mechanics and Dynamical Astronomy, 122:303-332, 2015.

[RSD99] R. M. Roberts e M. E. R. Sousa Dias. Symmetries of Riemann ellipsoids. Resenhas IME-USP, 4:183-221, 1999.

[TS02] D. L. Turcotte e G. Schubert. Geodynamics. Cambridge University Press, 2a edição, 2002.

[TT09] W. Thomson e P. G. Tait. Treatise on Natural Philosophy, volume 2. Cambridge University Press, New York, 2009. 